\title{
Initial and Boundary Value Problem for a System of Balance Laws from Chemotaxis: Global Dynamics and Diffusivity Limit
}

\author{
Zefu Feng ${ }^{1}$, Jiao $\mathrm{Xu}^{2}$, Ling $\mathrm{Xue}^{3}$ and Kun Zhao,** \\ 1 School of Mathematical Sciences, Chongqing Normal University, \\ Chongqing 400047, China \\ 2 SUSTech International Center for Mathematics, Southern University \\ of Science and Technology, Shenzhen 518055, Guangdong, China \\ ${ }^{3}$ College of Mathematical Sciences, Harbin Engineering University, \\ Harbin 150001, Heilongjiang, China \\ ${ }^{4}$ Department of Mathematics, Tulane University, New Orleans, \\ LA 70118, USA
}

Received 11 November 2020; Accepted (in revised version) 17 February 2021

\begin{abstract}
In this paper, we study long-time dynamics and diffusion limit of large-data solutions to a system of balance laws arising from a chemotaxis model with logarithmic sensitivity and nonlinear production/degradation rate. Utilizing energy methods, we show that under time-dependent Dirichlet boundary conditions, long-time dynamics of solutions are driven by their boundary data, and there is no restriction on the magnitude of initial energy. Moreover, the zero chemical diffusivity limit is established under zero Dirichlet boundary conditions, which has not been observed in previous studies on related models.
\end{abstract}

AMS subject classifications: 35B40, 35K51, 35Q92

Key words: Balance laws, chemotaxis, initial-boundary value problem, dynamic boundary condition, strong solution, long-time behavior, diffusivity limit.

*Corresponding author. Email addresses: zefufeng@mails.ccnu.edu.cn (Z. F. Feng), xuj7@sustech.edu.cn (J. Xu), lxue@hrbeu.edu.cn (L. Xue), kzhao@tulane.edu (K. Zhao) 


\section{Introduction}

\subsection{Overview}

This paper is oriented around the initial-boundary value problem:

$$
\begin{cases}p_{t}-(p q)_{x}=p_{x x}, & x \in(0,1), \quad t>0, \\ q_{t}-\left(p^{\gamma}+\varepsilon q^{2}\right)_{x}=\varepsilon q_{x x}, & x \in(0,1), \quad t>0, \\ (p, q)(x, 0)=\left(p_{0}, q_{0}\right)(x), & x \in(0,1), \\ \left.p\right|_{x=0,1}=\alpha(t),\left.\quad q\right|_{x=0,1}=\beta(t), & t>0, \quad \text { when } \varepsilon>0, \\ \left.p\right|_{x=0,1}=\alpha(t), & t>0, \quad \text { when } \varepsilon=0,\end{cases}
$$

where $\gamma>1$ and $\varepsilon$ are constant parameters, and $p_{0}(x), q_{0}(x), \alpha(t)$, and $\beta(t)$ are given functions. Assuming appropriate conditions for $p_{0}(x), q_{0}(x), \alpha(t)$ and $\beta(t)$, we establish global stability of strong solutions to (1.1). Moreover, when $\alpha(t)=\beta(t) \equiv 0$, we show that solutions to (1.1) with $\varepsilon>0$ converge to that with $\varepsilon=0$, as $\varepsilon \rightarrow 0$, in certain topology.

\section{$1.2 \quad$ Background}

The system of balance laws in problem (1.1) is derived from the following chemotaxis model of Keller-Segel type with logarithmic sensitivity:

$$
\left\{\begin{array}{l}
u_{t}=D u_{x x}-\chi\left(u(\ln c)_{x}\right)_{x} \\
c_{t}=\varepsilon c_{x x}-\mu \psi(u) c-\sigma c
\end{array}\right.
$$

when $\psi(u)=u^{\gamma}$. Here, the unknown functions $u(x, t)$ and $c(x, t)$ denote, respectively, density of cellular population and concentration of chemical signal at position $x$ at time $t$. The parameters: $D>0$ stands for diffusion coefficient of cellular density, $\chi \neq 0$ coefficient of chemotactic sensitivity, $\varepsilon \geq 0$ diffusion coefficient of chemical signal, $\mu \psi(u) \neq 0$ density-dependent production/degradation rate of chemical signal, $\psi(u)>0$ given function of cellular density, and $\sigma>0$ denotes natural degradation rate of chemical signal. The sign of $\chi$ dictates whether the chemotaxis is attractive $(\chi>0)$ or repulsive $(\chi<0)$, and $|\chi|$ measures the strength of chemotactic response. Moreover, the logarithmic sensitivity entails that the chemotactic response of cellular population to chemical signal follows Weber-Fechner's law (c.f. $[1,2,4,14]$ ), which appeared in one of the original Keller-Segel models of chemotaxis (c.f. [15]).

Derivation of the system of balance laws in (1.1) from (1.2) can be realized by 
first performing the following change of variables and rescaling:

$$
\begin{aligned}
& p=u, \quad q=\left[\ln \left(e^{\sigma t} c\right)\right]_{x}=\frac{c_{x}}{c}, \\
& t \rightarrow \frac{|\chi \mu|}{D} t, \quad x \rightarrow \frac{\sqrt{|\chi \mu|}}{D} x, \quad q \rightarrow-\operatorname{sign}(\chi) \sqrt{\frac{|\chi|}{|\mu|}} q,
\end{aligned}
$$

after which (1.2) becomes

$$
\left\{\begin{array}{l}
p_{t}-(p q)_{x}=p_{x x} \\
q_{t}-\left(\operatorname{sign}(\chi \mu) \psi(p)-\frac{\varepsilon}{\chi} q^{2}\right)_{x}=\frac{\varepsilon}{D} q_{x x} .
\end{array}\right.
$$

Then, taking $\chi=-1, D=1$ and $\mu<0$ in (1.3), one obtains

$$
\left\{\begin{array}{l}
p_{t}-(p q)_{x}=p_{x x} \\
q_{t}-\left(\psi(p)+\varepsilon q^{2}\right)_{x}=\varepsilon q_{x x}
\end{array}\right.
$$

Note that the case of $\chi<0$ and $\mu<0$ corresponds biologically to the chemotactic process in which the chemotaxis is repulsive and the chemical signal is being produced. Moreover, since the specific values of $\chi<0$ and $D>0$ do not affect the qualitative behavior of the solution, our choice of the parameter values is to simplify the presentation.

\subsection{Literature review}

In an effort of studying the chemotactic movement of organisms, such as myxobacteria, that deposit little- or non-diffusive chemical signals modifying succeed passages in local environment, Othmer-Stevens [29] and Levine-Sleeman [16] proposed the model:

$$
\left\{\begin{array}{l}
u_{t}=D u_{x x}-\chi\left(u(\ln c)_{x}\right)_{x} \\
c_{t}=-\mu u c-\sigma c
\end{array}\right.
$$

which corresponds to (1.2) when $\varepsilon=0$ and $\psi(u)=u$. Among the first generation of analytical studies of $(1.5)$, we refer the reader to $[5-7,18-20,37]$ for results concerning global well-posedness and large-time behavior of large-data solutions in one space dimension, and to $[13,21,22]$ for nonlinear stability of one-dimensional traveling wave solutions. tion):

Recently, the appended version of (1.5) (by adding diffusion to the second equa-

$$
\left\{\begin{array}{l}
u_{t}=D u_{x x}-\chi\left(u(\ln c)_{x}\right)_{x} \\
c_{t}=\varepsilon c_{x x}-\mu u c-\sigma c
\end{array}\right.
$$


has been investigated in a series of works $[8,19,23,24,28,30,33,36]$, where in addition to global well-posedness, large-time behavior of large-data solutions and nonlinear stability of traveling waves in one space dimension, the vanishing diffusivity limit (as $\varepsilon \rightarrow 0$ ) and boundary layer formation associated with one-dimensional solutions are studied. There are also similar results in multi-dimensional spaces, and we refer the reader to $[9,10,31,32,35]$ for details.

More recently, the authors of [38] studied another version of (1.2):

$$
\left\{\begin{array}{l}
u_{t}=D u_{x x}-\chi\left(u(\ln c)_{x}\right)_{x} \\
c_{t}=\varepsilon c_{x x}-\mu u^{\gamma} c-\sigma c
\end{array}\right.
$$

where $\gamma>1$ is a constant. Applying the same transformation and scaling as above to (1.7), one obtains the system of balance laws in (1.1). In [38], it is shown that the Cauchy problem of the transformed model possesses global (in time) large-data solutions with $\gamma$-dependent regularity, and the chemically diffusive $(\varepsilon>0)$ and nondiffusive $(\varepsilon=0)$ solutions are consistent in the limiting process as $\varepsilon \rightarrow 0$.

One of the major contributions of the results reported for the $1 \mathrm{D}$ models (1.5), (1.6) and (1.7) is the global stability of constant equilibrium solutions subject to various initial and/or boundary conditions. The proofs are constructed by utilizing $L^{p}$-based energy methods. One of the key ingredients consists in the implementation of entropy-entropy fluxes associated with the models, which is inspired by analytical techniques in hyperbolic conservation/balance laws.

It is also worth mentioning that very recently the following model:

$$
\left\{\begin{array}{l}
u_{t}=u_{x x}-\chi\left(u(\ln c)_{x}\right)_{x} \\
c_{t}=\varepsilon c_{x x}-u c^{m}, \quad m \geq 0
\end{array}\right.
$$

which is one of the original models proposed by Keller and Segel [15], is studied in [3]. Indeed, (1.6) is a special case of (1.8) when $m=1$. By considering the model (1.8) on the half line, $[0, \infty)$, and imposing zero-flux and Dirichlet boundary conditions at the left end-point for $u$ and $w$, respectively, and zero boundary condition at the far field, the authors studied the existence and stability of spike-layer solutions to the model. To the authors' knowledge, this is the first rigorous mathematical study dealing with the long-time asymptotic behavior of solutions to the model (1.8) when $m \neq 1$.

\subsection{Motivation and goal}

In this paper, we continue the analytical study of (1.7) by considering the model on a finite interval and studying the initial-boundary value problem (1.1) for the 
transformed model. A search in the database shows that such a problem has not been investigated in the literature. In real world, physical/biological processes always occur in bounded regions with constraints from boundaries, where initial-boundary value problems appear. Solutions to initial-boundary value problems usually exhibit different behaviors and much richer phenomena comparing with the Cauchy problem.

Our first work targets the long-time dynamics of large-data solutions to (1.1) when $\alpha(t)$ and $\beta(t)$ are non-trivial functions. One of the motivations of our study comes from the observation that boundary conditions oftentimes vary in time in many in vivo environments such as tumor angiogenesis (c.f. [30]). Since model (1.7) is deeply connected with (1.5) and (1.6) which have found applications in modeling tumor angiogenesis (see e.g., [17]), the problem considered in this paper becomes biologically relevant. Moreover, note that under dynamic boundary conditions, the initial-boundary value problem (1.1) can be reformulated as

$$
\left\{\begin{array}{l}
\tilde{p}_{t}-(\tilde{p} \tilde{q})_{x}=\tilde{p}_{x x}+\mathcal{F}_{1}, \\
\tilde{q}_{t}-\left(\psi(\tilde{p})+\varepsilon \tilde{q}^{2}\right)_{x}=\varepsilon \tilde{q}_{x x}+\mathcal{F}_{2}, \\
(\tilde{p}, \tilde{q})(x, 0)=\left(p_{0}-\alpha(0), q_{0}-\beta(0)\right)(x), \\
\left.\tilde{p}\right|_{x=0,1}=0,\left.\quad \tilde{q}\right|_{x=0,1}=0,
\end{array}\right.
$$

where $\tilde{p}=p-\alpha(t), \tilde{q}=q-\beta(t)$, and the feedback controls are given by $\mathcal{F}_{1}=\alpha(t) \tilde{q}_{x}+$ $\beta(t) \tilde{p}_{x}-\alpha^{\prime}(t)$ and $\mathcal{F}_{2}=2 \varepsilon \beta(t) \tilde{q}_{x}+(\psi(\tilde{p}+\alpha(t))-\psi(\tilde{p}))_{x}-\beta^{\prime}(t)$. Hence, the perturbed problem (1.9) can be viewed as a control problem of (1.1) subject to zero Dirichlet boundary conditions with control actions $\mathcal{F}_{1}$ and $\mathcal{F}_{2}$. As a typical question in control problem, one would ask: For what kind of feedback controls $\mathcal{F}_{1}$ and $\mathcal{F}_{2}$ does the closed-loop system (1.9) stabilize, i.e., its solution $(\tilde{p}, \tilde{q})$ tends to zero in an appropriate topology, as $t \rightarrow \infty$ ? We aim to give a definite answer to such a question in this paper.

The second goal of this paper is to investigate the zero chemical diffusivity limit of solutions to (1.1) when $p$ and $q$ satisfy the homogeneous Dirichlet boundary conditions. In all of the previous studies concerning initial-boundary value problems for model (1.6), it has been shown that if $p$ is supplemented with Dirichlet (including time-dependent) boundary conditions, then the transformed variable $q=\frac{c_{x}}{c}$ develops boundary layers at endpoints of the spatial interval as $\varepsilon \rightarrow 0$, see $[8,9,19,30]$. The inconsistency between chemically diffusive and non-diffusive solutions is caused by the mismatch of boundary conditions for the models with $\varepsilon>0$ and $\varepsilon=0$, respectively. Naturally, one would expect that the singular phenomenon also occurs to solutions to (1.1), no matter what condition $\alpha(t)$ is prescribed with. Indeed, by adopting the energy method in this paper and the idea of effective viscous flux in [30], one can show that as long as the boundary datum of $p$ is not identical to zero, boundary layers develop at endpoints of the spatial interval. On the other hand, however, we 
discovered that this is not the case when $p=0$ at $x=0$ and $x=1$. Roughly speaking, since $\left(p^{\gamma}\right)_{x}=\gamma p^{\gamma-1} p_{x}$, the zero boundary condition for $p$ generates the same boundary condition for $\left(p^{\gamma}\right)_{x}$ when $\gamma>1$. Hence, $q_{t}=0$ at the endpoints when $\varepsilon=0$. This is a strong indication, based on previous studies in [19,36], that the chemically diffusive solutions converge to the non-diffusive one as $\varepsilon \rightarrow 0$. Such a phenomenon is new in this specific research area, and we give it a rigorous demonstration in this paper.

\subsection{Statement of results}

Now we state the main results of this paper. The first two theorems demonstrate that large-data solutions to (1.1) stabilize as $t \rightarrow \infty$. The first theorem is concerned with the case when $\varepsilon>0$.

Theorem 1.1. Consider the initial-boundary value problem (1.1) when $\varepsilon>0$. Suppose that the initial data satisfy $p_{0}>0,\left(p_{0}, q_{0}\right) \in H^{1}((0,1))$ and are compatible with the boundary conditions. Assume that

- there exist constants $\underline{\alpha}, \bar{\alpha}, \bar{\beta}$, such that $0<\underline{\alpha} \leq \alpha(t) \leq \bar{\alpha}$ and $|\beta(t)| \leq \bar{\beta}$, for all $t \geq 0$

- $\left(\alpha^{\prime}(t), \beta^{\prime}(t)\right) \in L^{1}(0, \infty) \cap L^{2}(0, \infty)$.

Then for any fixed $\varepsilon>0$ and $\gamma>1$ there exists a unique global-in-time strong solution $(p, q)$, such that $(p-\alpha(t), q-\beta(t)) \in L^{\infty}\left(0, \infty ; H^{1}(0,1)\right) \cap L^{2}\left(0, \infty ; H^{2}(0,1)\right)$ and

$$
\lim _{t \rightarrow \infty}\left(\|p(\cdot, t)-\alpha(t)\|_{H^{1}}^{2}+\|q(\cdot, t)-\beta(t)\|_{H^{1}}^{2}\right)=0
$$

When $\varepsilon=0$, we have the following:

Theorem 1.2. Consider the initial-boundary value problem (1.1) when $\varepsilon=0$. Suppose that the initial data satisfy $p_{0}>0,\left(p_{0}, q_{0}\right) \in H^{1}((0,1))$ and are compatible with the boundary conditions. Assume that there exist constants $\underline{\alpha}, \bar{\alpha}$, such that $0<\underline{\alpha} \leq \alpha(t) \leq \bar{\alpha}$ for all $t \geq 0$, and $\alpha^{\prime}(t) \in L^{1}(0, \infty) \cap L^{2}(0, \infty)$. Then for any fixed $\gamma \geq 2$ there exists a unique global-in-time strong solution $(p, q)$, such that $(p-\alpha(t)) \in L^{\infty}\left(0, \infty ; H^{1}(0,1)\right) \cap$ $L^{2}\left(0, \infty ; H^{2}(0,1)\right),(q-\bar{q}) \in L^{\infty}\left(0, \infty ; H^{1}(0,1)\right) \cap L^{2}\left(0, \infty ; H^{1}(0,1)\right)$ and

$$
\lim _{t \rightarrow \infty}\left(\|p(\cdot, t)-\alpha(t)\|_{H^{1}}^{2}+\|q(\cdot, t)-\bar{q}\|_{H^{1}}^{2}\right)=0,
$$

where $\bar{q}$ denotes the spatial average of $q_{0}$.

We have several remarks regarding Theorem 1.1 and Theorem 1.2. 
Remark 1.1. The results recorded in Theorem 1.1 and Theorem 1.2 are the first ones regarding the transformed model in (1.1) on a bound domain with timedependent boundary conditions. One of the crucial assumptions in current work is that $p(0, t)=p(1, t)$ and $q(0, t)=q(1, t)$. From the physical point of view, it is more desirable to study the case when the unknown functions are subject to unmatched boundary values, i.e., $p(0, t)=\alpha_{0}(t), p(1, t)=\alpha_{1}(t), q(0, t)=\beta_{0}(t)$, and $q(1, t)=\beta_{1}(t)$, where $\alpha_{0}(t) \neq \alpha_{1}(t)$ and $\beta_{0}(t) \neq \beta_{1}(t)$. In this case, the underlying arguments will be significantly different, and more involved than those presented in this paper. The major obstruction lies in the fact that the original functions need to be perturbed around linear functions with respect to the spatial variable, i.e., $\alpha_{0}(t)+\left(\alpha_{1}(t)-\alpha_{0}(t)\right) x$ for $p(x, t)$ and $\beta_{0}(t)+\left(\beta_{1}(t)-\beta_{0}(t)\right) x$ for $q(x, t)$, in order to generate zero boundary values of the perturbed functions for subsequent energy estimates. In particular, the entropy estimate, see Subsection 2.1, must be revised to a great extent to accommodate the complex situation encountered in this more "realistic" situation. The detailed analysis is beyond the scope of this paper, and we leave the investigation in a future work.

Remark 1.2. The assumptions for $\alpha(t)$ and $\beta(t)$ in Theorem 1.1 and Theorem 1.2 imply that the boundary data converge to constants as time goes to infinity. By utilizing the energy methods in this paper, we can show that when there are no variations in the boundary data, i.e., constant Dirichlet boundary conditions, the perturbations converge exponentially rapidly to zero as time goes to infinity. In this case, quantities in the energy estimates involving derivatives of the boundary data vanish, and exponential decay of the perturbations follow from Poincaré's inequality. We omit the technical details to simplify the presentation.

Remark 1.3. Another set of boundary conditions that has been frequently studied for the transformed systems of the chemotaxis models (1.5) and (1.6) is the Neumann-Dirichlet boundary conditions, i.e., $\left.p_{x}\right|_{x=0,1}=0,\left.q\right|_{x=0,1}=0$. In this case, the spatial integral of $p$ is conserved. By adopting the energy methods in this paper, one can show that $(p, q)$ converges to $(\bar{p}, 0)$, where $\bar{p}$ denotes the spatial average of $p_{0}$, exponentially rapidly as time goes to infinity. One of the key ingredients in the proof is the fact that for any $t>0$, there exists $x_{t}^{*} \in(0,1)$, such that $p\left(x_{t}^{*}, t\right)=\bar{p}$, due to the conservation of the spatial integral of $p$. Such a fact enables one to implement Poincaré's inequality, leading to the exponential decaying of the perturbations. Moreover, by utilizing the energy framework in this paper and the idea of [38], we can show that under the Neumann-Dirichlet boundary conditions, the chemically diffusive solutions (i.e., $\varepsilon>0$ ) converge to the non-diffusive one (i.e., $\varepsilon=0$ ) in certain topology as $\varepsilon \rightarrow 0$.

The third theorem is concerned with the consistency between the chemically dif- 
fusive and non-diffusive solutions under the homogeneous Dirichlet boundary conditions, which has not been observed in previous studies for related models.

Theorem 1.3. Consider the initial-boundary value problem (1.1) with $\alpha(t)=\beta(t) \equiv 0$. Suppose that the initial data satisfy $p_{0}>0,\left(p_{0}, q_{0}\right) \in H^{1}((0,1))$ and are compatible with the boundary conditions. Then for any fixed $\gamma \geq 2$ and $\varepsilon \geq 0$, there exists a unique global-in-time strong solution $(p, q)$, such that for any $0<T<\infty$, $(p, q) \in L^{\infty}\left(0, T ; H^{1}(0,1)\right) \cap L^{2}\left(0, T ; H^{2}(0,1)\right)$ when $\varepsilon>0$, and $p \in L^{\infty}\left(0, T ; H^{1}(0,1)\right) \cap$ $L^{2}\left(0, T ; H^{2}(0,1)\right)$ and $q \in L^{\infty}\left(0, T ; H^{1}(0,1)\right) \cap L^{2}\left(0, T ; H^{1}(0,1)\right)$ when $\varepsilon=0$. Moreover, let $\left(p^{\varepsilon}, q^{\varepsilon}\right)$ and $\left(p^{0}, q^{0}\right)$ denote, respectively, the solution with $\varepsilon>0$ and $\varepsilon=0$, with the same initial data. Then for any $t>0$ it holds that

$$
\left\|\left(p^{\varepsilon}-p^{0}\right)(t)\right\|_{L^{2}}^{2}+\left\|\left(q^{\varepsilon}-p^{0}\right)(t)\right\|_{L^{2}}^{2} \leq C(t) \varepsilon,
$$

where the constant $C(t)>0$ is independent of $\varepsilon$ and remains finite for any finite $t>0$.

The rest of the paper is devoted to proof of Theorems 1.1-1.3. We utilize entropyand $L^{2}$-based energy methods. Similar to the proof constructed in [38], the point of departure is to implement an entropy-entropy flux pair associated with the system of balance laws in (1.1), which consists of the first order Taylor expansion of a power function of the unknown function $p$ around its boundary datum $\alpha$. The entropy estimate provides a uniform (with respect to $t$ ) bound of the $L^{2}$ norm of the perturbation around boundary data. However, the first order dissipation associated with $p$, generated from the entropy flux, may be degenerate (c.f. (2.14)). This is caused by the lack of a strictly positive a priori lower bound of $p$, which can not be achieved by the entropy estimate. To gain a non-degenerate dissipation mechanism of $p$, we then perform a series of nonlinear energy estimates. The detailed estimates differ case by case, depending on the value of $\gamma$. The proof is carefully crafted by examining some fine properties of power function. On the other hand, because of the time-dependency of the boundary data, the proof constructed in this paper is more involved than the one devised in [38] where the equilibrium solution is constant. We put a considerable amount of effort to make the energy framework developed in [38] fit into the complex situation encountered in this paper. Due to the structural difference between the chemically diffusive $(\varepsilon>0)$ and non-diffusive $(\varepsilon=0)$ problems, the proof of Theorem 1.1 is different from that of Theorem 1.2. Major difference consists in the estimation of the first order spatial derivative of the solution. For Theorem 1.1, the proof takes advantage of the positivity of chemical diffusivity, while for Theorem 1.2 we extract a non-homogeneous wave type equation for $q_{x}$, which serves as the foundation for deriving the desired energy estimates of the solution. Lastly, Theorem 1.3 is proved by utilizing the energy frameworks developed for Theorems 1.1-1.2 and exploring the zero boundary condition. 


\section{Proof of Theorem 1.1}

This section is denoted to the proof of Theorem 1.1. First of all, using standard arguments (see e.g., [25-27,34]), one can show that under the assumptions of Theorem 1.1 there exists a unique local solution to (1.1) with $\varepsilon>0$, such that $(p, q) \in L^{\infty}\left(0, T^{*} ; H^{1}(0,1)\right) \cap L^{2}\left(0, T^{*} ; H^{2}(0,1)\right)$ for some $T^{*} \in(0, \infty)$, and $p(x, t)>0$ for any $(x, t) \in(0,1) \times\left(0, T^{*}\right)$. The technical details are omitted to simplify the presentation. This section is largely devoted to deriving a priori estimates of the local solution, which not only extend the local solution to a global one, but also play an important role in investigating the global stability of the solution. The following technical lemmas are frequently utilized in the subsequent analysis (c.f. $[11,12,38])$.

Lemma 2.1. Let $a \geq-1$ and $\lambda \geq 2$. Then it holds that

$$
(a+1)^{\lambda}-1-\lambda a \geq \frac{\lambda}{2} a^{2} .
$$

Lemma 2.2. Let $a \geq-1$ and $\lambda \geq 2$. Then it holds that

$$
(a+1)^{\lambda}-1-\lambda a \geq|a|^{\lambda} .
$$

Lemma 2.3. Let $a \geq-1$ and $\lambda>1$. Then it holds that

$$
(a+1)^{\lambda} \geq 1+\lambda a .
$$

Lemma 2.4. Let $a \geq 0$ and $0<\lambda \leq 1$. Then it holds that

$$
\left|a^{\lambda}-1\right| \leq|a-1| \text {. }
$$

Next, we establish the a priori estimates of the local solution utilizing entropyand $L^{2}$-based energy methods.

Notation 2.1. Throughout the rest of the paper, unless otherwise specified, we use $C$ to denote a positive generic constant which is independent of the unknown functions and time. The value of the constant may vary line by line according to the context.

\section{$2.1 \quad$ Entropy estimate}

We first derive a uniform estimate based on the entropy-entropy flux pair associated with the initial-boundary value problem (1.1). For this purpose, let us define for any non-negative functions $f, g \in L^{\gamma}$,

$$
E(f, g)=\frac{1}{\gamma-1} \int_{0}^{1}\left[f^{\gamma}-g^{\gamma}-\gamma g^{\gamma-1}(f-g)\right] \mathrm{d} x .
$$

Since $\gamma>1$, then it follows from Taylor's theorem that $E(f, g) \geq 0$. 
Lemma 2.5. Under the assumptions of Theorem 1.1, there exists a constant $C>0$ which is independent of $t$ and $\varepsilon$, such that

$$
E(p, \alpha)+\|(q-\beta)(t)\|_{L^{2}}^{2}+\gamma \int_{0}^{t}\left\|p^{\frac{\gamma-2}{2}} p_{x}(\tau)\right\|_{L^{2}}^{2} \mathrm{~d} \tau+\varepsilon \int_{0}^{t}\left\|q_{x}(\tau)\right\|_{L^{2}}^{2} \mathrm{~d} \tau \leq C .
$$

Proof. We divide the proof into three steps.

Step 1. By a direct calculation, we can show that

$$
\left[p^{\gamma}-\alpha^{\gamma}-\gamma \alpha^{\gamma-1}(p-\alpha)\right]_{t}=\gamma\left(p^{\gamma-1}-\alpha^{\gamma-1}\right) p_{t}-\gamma \alpha^{\gamma-2}(p-\alpha) \alpha^{\prime}(t) .
$$

For the first term on the right-hand side of (2.2), using the first equation in (1.1) and noting that $\alpha$ is independent of $x$, we can show that

$$
\begin{aligned}
& \gamma\left(p^{\gamma-1}-\alpha^{\gamma-1}\right) p_{t} \\
= & \gamma\left(p^{\gamma-1}-\alpha^{\gamma-1}\right)\left[p_{x x}+(p q)_{x}\right] \\
= & \gamma\left[\left(p^{\gamma-1}-\alpha^{\gamma-1}\right)\left(p q+p_{x}\right)\right]_{x}-\gamma(\gamma-1) p^{\gamma-1} p_{x} q-\gamma(\gamma-1) p^{\gamma-2}\left(p_{x}\right)^{2} .
\end{aligned}
$$

Substituting (2.3) into (2.2), we deduce that

$$
\begin{aligned}
& {\left[p^{\gamma}-\alpha^{\gamma}-\gamma \alpha^{\gamma-1}(p-\alpha)\right]_{t} } \\
= & \gamma\left[\left(p^{\gamma-1}-\alpha^{\gamma-1}\right)\left(p q+p_{x}\right)\right]_{x}-\gamma(\gamma-1) p^{\gamma-1} p_{x} q \\
& -\gamma(\gamma-1) p^{\gamma-2}\left(p_{x}\right)^{2}-\gamma \alpha^{\gamma-2}(p-\alpha) \alpha^{\prime}(t) .
\end{aligned}
$$

Dividing (2.4) by $\gamma-1$, then integrating the result with respect to $x$ from 0 to 1 and using the boundary condition for $p$, we deduce

$$
\begin{aligned}
& \frac{\mathrm{d}}{\mathrm{d} t} E(p, \alpha)+\gamma \int_{0}^{1} p^{\gamma-2}\left(p_{x}\right)^{2} \mathrm{~d} x \\
= & -\gamma \int_{0}^{1} p^{\gamma-1} p_{x} q \mathrm{~d} x-\frac{\gamma \alpha^{\gamma-2}}{\gamma-1} \int_{0}^{1}(p-\alpha) \alpha^{\prime}(t) \mathrm{d} x .
\end{aligned}
$$

On the other hand, we derive from the second equation of (1.1) that

$$
(q-\beta)_{t}-\left(p^{\gamma}\right)_{x}=\varepsilon(q-\beta)_{x x}+2 \varepsilon(q-\beta)(q-\beta)_{x}+2 \varepsilon \beta(q-\beta)_{x}-\beta^{\prime}(t) .
$$

Taking $L^{2}$ inner product of (2.6) with $q-\beta$ and using the boundary conditions yield

$$
\begin{aligned}
& \frac{1}{2} \frac{\mathrm{d}}{\mathrm{d} t}\|q-\beta\|_{L^{2}}^{2}+\varepsilon\left\|(q-\beta)_{x}\right\|_{L^{2}}^{2} \\
= & \int_{0}^{1}(q-\beta)\left(p^{\gamma}\right)_{x} \mathrm{~d} x-\int_{0}^{1}(q-\beta) \beta^{\prime}(t) \mathrm{d} x \\
= & \int_{0}^{1} q\left(p^{\gamma}\right)_{x} \mathrm{~d} x-\int_{0}^{1}(q-\beta) \beta^{\prime}(t) \mathrm{d} x \\
= & \gamma \int_{0}^{1} p^{\gamma-1} p_{x} q \mathrm{~d} x-\int_{0}^{1}(q-\beta) \beta^{\prime}(t) \mathrm{d} x .
\end{aligned}
$$


Adding (2.7) to (2.5), we obtain

$$
\begin{aligned}
& \frac{\mathrm{d}}{\mathrm{d} t}\left(E(p, \alpha)+\frac{1}{2}\|q-\beta\|_{L^{2}}^{2}\right)+\gamma \int_{0}^{1} p^{\gamma-2}\left(p_{x}\right)^{2} \mathrm{~d} x+\varepsilon\left\|(q-\beta)_{x}\right\|_{L^{2}}^{2} \\
= & -\frac{\gamma}{\gamma-1} \int_{0}^{1} \alpha^{\gamma-2}(p-\alpha) \alpha^{\prime}(t) \mathrm{d} x-\int_{0}^{1}(q-\beta) \beta^{\prime}(t) \mathrm{d} x .
\end{aligned}
$$

Step 2. In this step, we derive a bound for the $L^{1}$ norm of $p$ in terms of $E(p, \alpha)$. The idea is to make use of the convexity of the entropy expansion, $E(p, \alpha)$, and compare it with a linear function of $p$. For this purpose, we set

$$
F_{\alpha}(p)=\frac{1}{\gamma-1}\left[p^{\gamma}-\alpha^{\gamma}-\gamma \alpha^{\gamma-1}(p-\alpha)\right]+\left(\alpha^{\gamma-1}+\frac{\gamma-1}{\gamma}\right)^{\frac{1}{\gamma-1}}-p
$$

By direct calculations, we can show that

$$
\begin{aligned}
& F_{\alpha}(0)=\alpha^{\gamma}+\left(\alpha^{\gamma-1}+\frac{\gamma-1}{\gamma}\right)^{\frac{1}{\gamma-1}}>0, \\
& F_{\alpha}^{\prime}\left(\left(\alpha^{\gamma-1}+\frac{\gamma-1}{\gamma}\right)^{\frac{1}{\gamma-1}}\right)=0, \\
& F_{\alpha}^{\prime \prime}(p)=\gamma p^{\gamma-2}>0
\end{aligned}
$$

for $p>0, \alpha>0$. Moreover, since

$$
p^{\gamma}-\alpha^{\gamma}-\gamma \alpha^{\gamma-1}(p-\alpha) \geq 0
$$

for any $p>0, \alpha>0$, we have

$$
F_{\alpha}\left(\left(\alpha^{\gamma-1}+\frac{\gamma-1}{\gamma}\right)^{\frac{1}{\gamma-1}}\right) \geq 0
$$

These imply that $F_{\alpha}(p) \geq 0$ for any $p>0, \alpha>0$. Hence, it holds that

$$
0<p \leq \frac{1}{\gamma-1}\left[p^{\gamma}-\alpha^{\gamma}-\gamma \alpha^{\gamma-1}(p-\alpha)\right]+\left(\alpha^{\gamma-1}+\frac{\gamma-1}{\gamma}\right)^{\frac{1}{\gamma-1}}
$$

and therefore,

$$
0<\int_{0}^{1} p(x, t) \mathrm{d} x \leq E(p, \alpha)+\left(\alpha^{\gamma-1}+\frac{\gamma-1}{\gamma}\right)^{\frac{1}{\gamma-1}}
$$


Step 3. Utilizing (2.9), we derive from (2.7) that

$$
\begin{aligned}
& \frac{\mathrm{d}}{\mathrm{d} t}\left(E(p, \alpha)+\frac{1}{2}\|q-\beta\|_{L^{2}}^{2}\right)+\gamma \int_{0}^{1} p^{\gamma-2}\left(p_{x}\right)^{2} \mathrm{~d} x+\varepsilon\left\|(q-\beta)_{x}\right\|_{L^{2}}^{2} \\
= & -\frac{\gamma}{\gamma-1} \int_{0}^{1} \alpha^{\gamma-2}(p-\alpha) \alpha^{\prime}(t) \mathrm{d} x-\int_{0}^{1}(q-\beta) \beta^{\prime}(t) \mathrm{d} x \\
\leq & \frac{\gamma}{\gamma-1} \alpha^{\gamma-2}\left|\alpha^{\prime}(t)\right| \int_{0}^{1}|p-\alpha| \mathrm{d} x+\left|\beta^{\prime}(t)\right| \int_{0}^{1}|q-\beta| \mathrm{d} x \\
\leq & \frac{\gamma}{\gamma-1} \alpha^{\gamma-2}\left|\alpha^{\prime}(t)\right| E(p, \alpha)+\frac{\gamma}{\gamma-1} \alpha^{\gamma-2}\left|\alpha^{\prime}(t)\right|\left(\alpha^{\gamma-1}+\frac{\gamma-1}{\gamma}\right)^{\frac{1}{\gamma-1}} \\
& \quad+\frac{\gamma}{\gamma-1} \alpha^{\gamma-1}\left|\alpha^{\prime}(t)\right|+\frac{\left|\beta^{\prime}(t)\right|}{2}+\frac{\left|\beta^{\prime}(t)\right|}{2}\|q-\beta\|_{L^{2}}^{2} .
\end{aligned}
$$

Using the assumptions in Theorem 1.1 and applying Grönwall's inequality to (2.10), we have

$$
\begin{aligned}
& \quad E(p, \alpha)+\frac{1}{2}\|q-\beta\|_{L^{2}}^{2} \\
& \leq \exp \left\{\int_{0}^{t}\left(\frac{\gamma}{\gamma-1} \bar{\alpha}^{\gamma-2}\left|\alpha^{\prime}(\tau)\right|+\left|\beta^{\prime}(\tau)\right|\right) \mathrm{d} \tau\right\} \\
& \quad \times\left[\int_{0}^{t}\left(\frac{\gamma}{\gamma-1} \bar{\alpha}^{\gamma-2}\left|\alpha^{\prime}(\tau)\right|\left(\bar{\alpha}^{\gamma-1}+\frac{\gamma-1}{\gamma}\right)^{\frac{1}{\gamma-1}}+\frac{\gamma}{\gamma-1} \bar{\alpha}^{\gamma-1}\left|\alpha^{\prime}(\tau)\right|+\frac{\left|\beta^{\prime}(\tau)\right|}{2}\right) \mathrm{d} \tau\right. \\
& \left.\quad+E\left(p_{0}, \alpha_{0}\right)+\frac{1}{2}\left\|q_{0}-\beta_{0}\right\|_{L^{2}}^{2}\right] \\
& \leq C, \quad \forall t>0, \quad \forall \varepsilon>0 .
\end{aligned}
$$

Substituting (2.11) into (2.10), then integrating the result with respect to $t$, we have

$$
\gamma \int_{0}^{t} \int_{0}^{1} p^{\gamma-2}\left(p_{x}\right)^{2} \mathrm{~d} x \mathrm{~d} \tau+\varepsilon \int_{0}^{t}\left\|(q-\beta)_{x}\right\|_{L^{2}}^{2} \mathrm{~d} \tau \leq C, \quad \forall t>0, \quad \forall \varepsilon>0 .
$$

Combining (2.11) and (2.12) completes the proof of Lemma 2.5.

Next, we derive the key estimate in this paper.

\section{$2.2 \quad L^{2}$ estimate}

Letting $\tilde{p}=p-\alpha, \tilde{q}=q-\beta$, the IBVP (1.1) is reformulated as

$$
\begin{cases}\tilde{p}_{t}-(\tilde{p} \tilde{q})_{x}-\alpha \tilde{q}_{x}-\beta \tilde{p}_{x}=\tilde{p}_{x x}-\alpha^{\prime}(t), & x \in(0,1), \quad t>0, \\ \tilde{q}_{t}-(\tilde{p}+\alpha)_{x}^{\gamma}=\varepsilon \tilde{q}_{x x}+2 \varepsilon \tilde{q} \tilde{q}_{x}+2 \varepsilon \beta \tilde{q}_{x}-\beta^{\prime}(t), & x \in(0,1), \quad t>0, \\ (\tilde{p}, \tilde{q})(x, 0)=\left(p_{0}-\alpha(0), q_{0}-\beta(0)\right)(x), & x \in(0,1), \\ \left.\tilde{p}\right|_{x=0,1}=0,\left.\quad \tilde{q}\right|_{x=0,1}=0, & t>0 .\end{cases}
$$


In terms of the perturbed functions, (2.1) is rewritten as

$$
\widetilde{E}(\tilde{p}, \alpha)+\frac{1}{2}\|\tilde{q}\|_{L^{2}}^{2}+\gamma \int_{0}^{t} \int_{0}^{1}(\tilde{p}+\alpha)^{\gamma-2}\left(\tilde{p}_{x}\right)^{2} \mathrm{~d} x \mathrm{~d} \tau+\varepsilon \int_{0}^{t}\left\|\tilde{q}_{x}\right\|_{L^{2}}^{2} \mathrm{~d} \tau \leq C,
$$

where

$$
\widetilde{E}(\tilde{p}, \alpha)=E(\tilde{p}+\alpha, \alpha)=\frac{1}{\gamma-1} \int_{0}^{1}\left[(\tilde{p}+\alpha)^{\gamma}-\alpha^{\gamma}-\gamma \alpha^{\gamma-1} \tilde{p}\right] \mathrm{d} x
$$

Note that when $\gamma \geq 2$, letting $a=\frac{\tilde{p}}{\alpha}$ in Lemma 2.1, we obtain

$$
(\tilde{p}+\alpha)^{\gamma}-\alpha^{\gamma}-\gamma \alpha^{\gamma-1} \tilde{p} \geq \frac{\gamma}{2} \alpha^{\gamma-2} \tilde{p}^{2} \geq \frac{\gamma}{2} \underline{\alpha}^{\gamma-2} \tilde{p}^{2}
$$

which implies

$$
\widetilde{E}(\tilde{p}, \alpha) \geq \frac{\gamma}{2(\gamma-1)} \underline{\alpha}^{\gamma-2}\|\tilde{p}\|_{L^{2}}^{2}
$$

Moreover, letting $a=\frac{\tilde{p}}{\alpha}$ in Lemma 2.2, one has

$$
\widetilde{E}(\tilde{p}, \alpha) \geq \frac{1}{\gamma-1} \int_{0}^{1}|\tilde{p}|^{\gamma} \mathrm{d} x=\frac{1}{\gamma-1}\|\tilde{p}\|_{L^{\gamma}}^{\gamma} .
$$

In light of (2.14), (2.17) and (2.18), we see that

$$
\|\tilde{p}(t)\|_{L^{\gamma}}^{\gamma} \leq(\gamma-1) C, \quad\|\tilde{p}(t)\|_{L^{2}}^{2} \leq \frac{2(\gamma-1) C}{\gamma \underline{\alpha}^{\gamma-2}}, \quad \gamma \geq 2 .
$$

The estimates in (2.19) are frequently utilized in the subsequent proof. Note that the first $L_{t}^{2} L_{x}^{2}$ estimate in (2.1) may be degenerate since we do not have a strictly positive lower bound of $p$. Next, we derive a non-degenerate estimate of $\|\tilde{p}\|_{L_{t}^{2} L_{x}^{2}}$.

Lemma 2.6. Under the conditions of Theorem 1.1 for any $\gamma>1, \varepsilon>0$, and $t>0$, there exists a positive constant $C$, which is independent of $t$ and $\varepsilon$ such that

$$
\begin{cases}\int_{0}^{t}\left(\left\|\tilde{p} \tilde{p}_{x}(\tau)\right\|_{L^{2}}^{2}+\left\|\tilde{p}_{x}(\tau)\right\|_{L^{2}}^{2}\right) \mathrm{d} \tau \leq C, & \gamma \in(1,4] \\ \int_{0}^{t}\left(\int_{0}^{1}|\tilde{p}|^{\gamma-1}\left(\tilde{p}_{x}\right)^{2} \mathrm{~d} x+\left\|\tilde{p}_{x}(\tau)\right\|_{L^{2}}^{2}\right) \mathrm{d} \tau \leq C, & \gamma>4\end{cases}
$$

We divide the proof of Lemma 2.6 into four sub-cases, namely, $1<\gamma<2,2 \leq \gamma \leq 3$, $3<\gamma \leq 4$, and $\gamma>4$, since the technical details differ case by case. 


\subsubsection{Proof of Lemma 2.6 when $1<\gamma<2$}

Step 1. Multiplying the first equation of (2.13) by $(\tilde{p}+\alpha)^{\gamma}-\alpha^{\gamma}$ and the second equation by $\alpha \tilde{q}$, after integrating by parts, we obtain

$$
\begin{aligned}
& \frac{\mathrm{d}}{\mathrm{d} t}\left(\frac{1}{\gamma+1} \int_{0}^{1} \widehat{E}(\tilde{p}, \alpha) \mathrm{d} x+\frac{\alpha}{2}\|\tilde{q}\|_{L^{2}}^{2}\right)+\gamma \int_{0}^{1}(\tilde{p}+\alpha)^{\gamma-1}\left(\tilde{p}_{x}\right)^{2} \mathrm{~d} x+\varepsilon \alpha\left\|\tilde{q}_{x}\right\|_{L^{2}}^{2} \\
= & -\gamma \int_{0}^{1}(\tilde{p}+\alpha)^{\gamma-1} \tilde{p} \tilde{q} \tilde{p}_{x} \mathrm{~d} x-\gamma \alpha^{\gamma-1} \alpha^{\prime}(t) \int_{0}^{1} \tilde{p} \mathrm{~d} x-\alpha \beta^{\prime}(t) \int_{0}^{1} \tilde{q} \mathrm{~d} x+\frac{\alpha^{\prime}(t)}{2}\|\tilde{q}\|_{L^{2}}^{2},
\end{aligned}
$$

where

$$
\widehat{E}(\tilde{p}, \alpha)=(\tilde{p}+\alpha)^{\gamma+1}-\alpha^{\gamma+1}-(\gamma+1) \alpha^{\gamma} \tilde{p} .
$$

Since $1<\gamma<2$, by choosing $a=\frac{\tilde{p}}{\alpha}$ in Lemma 2.1 , we can show that

$$
\widehat{E}(\tilde{p}, \alpha) \geq \frac{\gamma+1}{2} \alpha^{\gamma-1}|\tilde{p}|^{2}
$$

which implies

$$
\frac{1}{\gamma+1} \int_{0}^{1} \widehat{E}(\tilde{p}, \alpha) \mathrm{d} x \geq \frac{\alpha^{\gamma-1}}{2}\|\tilde{p}\|_{L^{2}}^{2} \geq \frac{\underline{\alpha}^{\gamma-1}}{2}\|\tilde{p}\|_{L^{2}}^{2} .
$$

Step 2. Multiplying the first equation of (2.13) by $-\frac{\gamma}{2} \alpha^{\gamma-2} \tilde{p}^{2}$, then integrating by parts, we can show that

$$
\begin{aligned}
& \frac{\mathrm{d}}{\mathrm{d} t}\left(-\frac{\gamma}{6} \alpha^{\gamma-2} \int_{0}^{1} \tilde{p}^{3} \mathrm{~d} x\right)-\gamma \alpha^{\gamma-2} \int_{0}^{1} \tilde{p}\left(\tilde{p}_{x}\right)^{2} \mathrm{~d} x \\
= & \gamma \alpha^{\gamma-2} \int_{0}^{1} \tilde{p}^{2} \tilde{q} \tilde{p}_{x} \mathrm{~d} x+\gamma \alpha^{\gamma-1} \int_{0}^{1} \tilde{p} \tilde{q} \tilde{p}_{x} \mathrm{~d} x \\
& +\frac{\gamma}{2} \alpha^{\gamma-2} \alpha^{\prime}(t) \int_{0}^{1} \tilde{p}^{2} \mathrm{~d} x-\frac{\gamma(\gamma-2)}{6} \alpha^{\gamma-3} \alpha^{\prime}(t) \int_{0}^{1} \tilde{p}^{3} \mathrm{~d} x .
\end{aligned}
$$

Multiplying the first equation of $(2.13)$ by $\frac{\gamma}{3} \alpha^{\gamma-3} \tilde{p}^{3}$, then integrating by parts, we can show that

$$
\begin{aligned}
& \frac{\mathrm{d}}{\mathrm{d} t}\left(\frac{\gamma}{12} \alpha^{\gamma-3} \int_{0}^{1} \tilde{p}^{4} \mathrm{~d} x\right)+\gamma \alpha^{\gamma-3} \int_{0}^{1}\left(\tilde{p} \tilde{p}_{x}\right)^{2} \mathrm{~d} x \\
= & -\gamma \alpha^{\gamma-3} \int_{0}^{1} \tilde{p}^{3} \tilde{q} \tilde{p}_{x} \mathrm{~d} x-\gamma \alpha^{\gamma-2} \int_{0}^{1} \tilde{p}^{2} \tilde{q} \tilde{p}_{x} \mathrm{~d} x \\
& \quad-\frac{\gamma}{3} \alpha^{\gamma-3} \alpha^{\prime}(t) \int_{0}^{1} \tilde{p}^{3} \mathrm{~d} x+\frac{\gamma(\gamma-3)}{12} \alpha^{\gamma-4} \alpha^{\prime}(t) \int_{0}^{1} \tilde{p}^{4} \mathrm{~d} x .
\end{aligned}
$$


Combining (4.2), (2.24) and (2.25), we have

$$
\frac{\mathrm{d}}{\mathrm{d} t} G(t)+H(t)=K(t)
$$

where

$$
\begin{aligned}
& G(t)=\frac{1}{\gamma+1} \int_{0}^{1} \widehat{E}(\tilde{p}, \alpha) \mathrm{d} x+\frac{\alpha}{2}\|\tilde{q}\|_{L^{2}}^{2}-\frac{\gamma}{6} \alpha^{\gamma-2} \int_{0}^{1} \tilde{p}^{3} \mathrm{~d} x+\frac{\gamma}{12} \alpha^{\gamma-3} \int_{0}^{1} \tilde{p}^{4} \mathrm{~d} x \\
& H(t)=\gamma \int_{0}^{1}(\tilde{p}+\alpha)^{\gamma-1}\left(\tilde{p}_{x}\right)^{2} \mathrm{~d} x+\varepsilon \alpha\left\|\tilde{q}_{x}\right\|_{L^{2}}^{2}-\gamma \alpha^{\gamma-2} \int_{0}^{1} \tilde{p}\left(\tilde{p}_{x}\right)^{2} \mathrm{~d} x+\gamma \alpha^{\gamma-3} \int_{0}^{1}\left(\tilde{p} \tilde{p}_{x}\right)^{2} \mathrm{~d} x, \\
& K(t)=-\gamma \int_{0}^{1}\left[(\tilde{p}+\alpha)^{\gamma-1}-\alpha^{\gamma-1}\right] \tilde{p} \tilde{q} \tilde{p}_{x} \mathrm{~d} x-\gamma \alpha^{\gamma-3} \int_{0}^{1} \tilde{p}^{3} \tilde{q} \tilde{p}_{x} \mathrm{~d} x-\gamma \alpha^{\gamma-1} \alpha^{\prime}(t) \int_{0}^{1} \tilde{p} \mathrm{~d} x \\
& \quad-\alpha \beta^{\prime}(t) \int_{0}^{1} \tilde{q} \mathrm{~d} x+\frac{\alpha^{\prime}(t)}{2}\|\tilde{q}\|_{L^{2}}^{2}+\frac{\gamma}{2} \alpha^{\gamma-2} \alpha^{\prime}(t) \int_{0}^{1} \tilde{p}^{2} \mathrm{~d} x-\frac{\gamma^{2}}{6} \alpha^{\gamma-3} \alpha^{\prime}(t) \int_{0}^{1} \tilde{p}^{3} \mathrm{~d} x \\
& \quad+\frac{\gamma(\gamma-3)}{12} \alpha^{\gamma-4} \alpha^{\prime}(t) \int_{0}^{1} \tilde{p}^{4} \mathrm{~d} x .
\end{aligned}
$$

Step 3. We have the following observations regarding $G(t), H(t)$ and $K(t)$. First, by virtue of (2.23), we can show that

$$
\begin{aligned}
G(t) & \geq \frac{\alpha^{\gamma-1}}{2} \int_{0}^{1} \tilde{p}^{2} \mathrm{~d} x-\frac{\gamma}{6} \alpha^{\gamma-2} \int_{0}^{1} \tilde{p}^{3} \mathrm{~d} x+\frac{\gamma}{12} \alpha^{\gamma-3} \int_{0}^{1} \tilde{p}^{4} \mathrm{~d} x+\frac{\alpha}{2}\|\tilde{q}\|_{L^{2}}^{2} \\
& =\frac{(3-\gamma)}{6} \alpha^{\gamma-1}\|\tilde{p}\|_{L^{2}}^{2}+\frac{\gamma}{24} \alpha^{\gamma-3}\left\|2 \alpha \tilde{p}-\tilde{p}^{2}\right\|_{L^{2}}^{2}+\frac{\gamma}{24} \alpha^{\gamma-3}\|\tilde{p}\|_{L^{4}}^{4}+\frac{\alpha}{2}\|\tilde{q}\|_{L^{2}}^{2}
\end{aligned}
$$

For $H(t)$, by a direct calculation, we can show that

$$
\begin{aligned}
H(t)=\gamma & \int_{0}^{1}\left[(\tilde{p}+\alpha)^{\gamma-1}-\alpha^{\gamma-1}\right]\left(\tilde{p}_{x}\right)^{2} \mathrm{~d} x+\frac{\gamma}{2} \alpha^{\gamma-1}\left\|\tilde{p}_{x}\right\|_{L^{2}}^{2}+\frac{\gamma}{2} \alpha^{\gamma-3}\left\|\alpha \tilde{p}_{x}-\tilde{p} \tilde{p}_{x}\right\|_{L^{2}}^{2} \\
& +\frac{\gamma}{2} \alpha^{\gamma-3}\left\|\tilde{p} \tilde{p}_{x}\right\|_{L^{2}}^{2}+\varepsilon \alpha\left\|\tilde{q}_{x}\right\|_{L^{2}}^{2} .
\end{aligned}
$$

Using Lemma 2.4 and the Cauchy-Schwarz inequality, we can show that

$$
(\tilde{p}+\alpha)^{\gamma-1}-\alpha^{\gamma-1} \geq-\alpha^{\gamma-2}|\tilde{p}| \geq-\alpha^{\gamma-3}(\tilde{p})^{2}-\frac{1}{4} \alpha^{\gamma-1} .
$$

Using (2.29), we update (2.28) as

$$
H(t) \geq \frac{\gamma}{4} \alpha^{\gamma-1}\left\|\tilde{p}_{x}\right\|_{L^{2}}^{2}+\frac{\gamma}{2} \alpha^{\gamma-3}\left\|\alpha \tilde{p}_{x}-\tilde{p} \tilde{p}_{x}\right\|_{L^{2}}^{2}-\frac{\gamma}{2} \alpha^{\gamma-3}\left\|\tilde{p} \tilde{p}_{x}\right\|_{L^{2}}^{2}+\varepsilon \alpha\left\|\tilde{q}_{x}\right\|_{L^{2}}^{2} .
$$


Since $0<\underline{\alpha} \leq \alpha \leq \bar{\alpha}$, using the Cauchy-Schwarz inequality, we can show that

$$
\begin{aligned}
K(t) \leq- & \gamma \int_{0}^{1}\left[(\tilde{p}+\alpha)^{\gamma-1}-\alpha^{\gamma-1}\right] \tilde{p} \tilde{q} \tilde{p}_{x} \mathrm{~d} x-\gamma \alpha^{\gamma-3} \int_{0}^{1} \tilde{p}^{3} \tilde{q} \tilde{p}_{x} \mathrm{~d} x \\
& +\frac{\gamma}{2} \bar{\alpha}^{\gamma-1}\left|\alpha^{\prime}(t)\right|\left(\|\tilde{p}\|_{L^{2}}^{2}+1\right)+\frac{\bar{\alpha}}{2}\left|\beta^{\prime}(t)\right|\left(\|\tilde{q}\|_{L^{2}}^{2}+1\right)+\frac{\left|\alpha^{\prime}(t)\right|}{2}\|\tilde{q}\|_{L^{2}}^{2} \\
& +\frac{\gamma}{2} \underline{\alpha}^{\gamma-2}\left|\alpha^{\prime}(t)\right|\|\tilde{p}\|_{L^{2}}^{2}+\frac{\gamma^{2}}{12} \underline{\alpha}^{\gamma-3}\left|\alpha^{\prime}(t)\right|\left(\|\tilde{p}\|_{L^{4}}^{4}+\|\tilde{p}\|_{L^{2}}^{2}\right) \\
& +\frac{\gamma(3-\gamma)}{12} \underline{\alpha}^{\gamma-4}\left|\alpha^{\prime}(t)\right|\|\tilde{p}\|_{L^{4}}^{4} .
\end{aligned}
$$

In view of (2.27), we see that there exists a generic constant $C>0$, such that

$$
\begin{aligned}
K(t) \leq- & \gamma \int_{0}^{1}\left[(\tilde{p}+\alpha)^{\gamma-1}-\alpha^{\gamma-1}\right] \tilde{p} \tilde{q} \tilde{p}_{x} \mathrm{~d} x-\gamma \alpha^{\gamma-3} \int_{0}^{1} \tilde{p}^{3} \tilde{q} \tilde{p}_{x} \mathrm{~d} x \\
& +C\left(\left|\alpha^{\prime}(t)\right|+\left|\beta^{\prime}(t)\right|\right) G(t)+C\left(\left|\alpha^{\prime}(t)\right|+\left|\beta^{\prime}(t)\right|\right) .
\end{aligned}
$$

Step 4. We note that the third term on the right-hand side of (2.30) is non-positive. To compensate such a term, we add (2.25) to (2.26) to get

$$
\begin{aligned}
& \frac{\mathrm{d}}{\mathrm{d} t} X(t)+Y(t) \\
\leq- & \gamma \int_{0}^{1}\left[(\tilde{p}+\alpha)^{\gamma-1}-\alpha^{\gamma-1}\right] \tilde{p} \tilde{q} \tilde{p}_{x} \mathrm{~d} x-2 \gamma \alpha^{\gamma-3} \int_{0}^{1} \tilde{p}^{3} \tilde{q} \tilde{p}_{x} \mathrm{~d} x \\
& \quad-\gamma \alpha^{\gamma-2} \int_{0}^{1} \tilde{p}^{2} \tilde{q} \tilde{p}_{x} \mathrm{~d} x+C\left(\left|\alpha^{\prime}(t)\right|+\left|\beta^{\prime}(t)\right|\right) X(t)+C\left(\left|\alpha^{\prime}(t)\right|+\left|\beta^{\prime}(t)\right|\right),
\end{aligned}
$$

where we used (2.32) and arguments similar to those in (2.31), and

$$
\begin{aligned}
& X(t)=G(t)+\frac{\gamma}{12} \alpha^{\gamma-3} \int_{0}^{1} \tilde{p}^{4} \mathrm{~d} x, \\
& Y(t)=H(t)+\gamma \alpha^{\gamma-3}\left\|\tilde{p} \tilde{p}_{x}\right\|_{L^{2}}^{2} .
\end{aligned}
$$

According to (2.30), we have

$$
Y(t) \geq \frac{\gamma}{4} \alpha^{\gamma-1}\left\|\tilde{p}_{x}\right\|_{L^{2}}^{2}+\frac{\gamma}{2} \alpha^{\gamma-3}\left\|\alpha \tilde{p}_{x}-\tilde{p} \tilde{p}_{x}\right\|_{L^{2}}^{2}+\frac{\gamma}{2} \alpha^{\gamma-3}\left\|\tilde{p} \tilde{p}_{x}\right\|_{L^{2}}^{2}+\varepsilon \alpha\left\|\tilde{q}_{x}\right\|_{L^{2}}^{2} .
$$

For the first term on the right-hand side of (2.33), due to Lemma 2.4, it holds that

$$
\left|(\tilde{p}+\alpha)^{\gamma-1}-\alpha^{\gamma-1}\right| \leq \alpha^{\gamma-2}|\tilde{p}|,
$$


by using which we update (2.33) as

$$
\begin{aligned}
\frac{\mathrm{d}}{\mathrm{d} t} X(t)+Y(t) \leq \underbrace{2 \gamma \alpha^{\gamma-2} \int_{0}^{1}|\tilde{p}|^{2}|\tilde{q}|\left|\tilde{p}_{x}\right| \mathrm{d} x}_{\equiv J_{1}} & +\underbrace{2 \gamma \alpha^{\gamma-3} \int_{0}^{1}|\tilde{p}|^{3}|q|\left|\tilde{p}_{x}\right| \mathrm{d} x}_{\equiv J_{2}} \\
+C\left(\left|\alpha^{\prime}(t)\right|+\left|\beta^{\prime}(t)\right|\right) X(t)+C\left(\left|\alpha^{\prime}(t)\right|+\left|\beta^{\prime}(t)\right|\right) . &
\end{aligned}
$$

For the first two terms on the right-hand side of (2.36), by using the same method [38, Lemma 5.1], we can show that

$$
J_{1} \leq C\|\tilde{p}\|_{L^{2}}^{2}\left(\int_{0}^{1}(\tilde{p}+\alpha)^{\gamma-2}\left(\tilde{p}_{x}\right)^{2} \mathrm{~d} x\right)+\frac{\gamma}{16} \alpha^{\gamma-1}\left\|\tilde{p}_{x}\right\|_{L^{2}}^{2}+\frac{\gamma}{8} \alpha^{\gamma-3}\left\|\tilde{p} \tilde{p}_{x}\right\|_{L^{2}}^{2},
$$

and

$$
J_{2} \leq C\|\tilde{p}\|_{L^{4}}^{4}\left(\int_{0}^{1}(\tilde{p}+\alpha)^{\gamma-2}\left(\tilde{p}_{x}\right)^{2} \mathrm{~d} x\right)+\frac{\gamma}{16} \alpha^{\gamma-1}\left\|\tilde{p}_{x}\right\|_{L^{2}}^{2}+\frac{\gamma}{8} \alpha^{\gamma-3}\left\|\tilde{p} \tilde{p}_{x}\right\|_{L^{2}}^{2} .
$$

Using (2.38) and (2.37), we update (2.36) as

$$
\begin{aligned}
\frac{\mathrm{d}}{\mathrm{d} t} X(t)+\frac{1}{2} Y(t) \leq C & \left(\left|\alpha^{\prime}(t)\right|+\left|\beta^{\prime}(t)\right|++C \int_{0}^{1}(\tilde{p}+\alpha)^{\gamma-2}\left(\tilde{p}_{x}\right)^{2} \mathrm{~d} x\right) X(t) \\
& +C\left(\left|\alpha^{\prime}(t)\right|+\left|\beta^{\prime}(t)\right|\right) .
\end{aligned}
$$

Applying Grönwall's inequality to (2.39) and using (2.12) and the assumptions in Theorem 1.1, we can show that

$$
X(t)+\int_{0}^{t} Y(\tau) \mathrm{d} \tau \leq C .
$$

According to (2.27), the definition of $X(t)$, and (2.34), we conclude that

$$
\|\tilde{p}\|_{L^{2}}^{2}+\|\tilde{p}\|_{L^{4}}^{4}+\|\tilde{q}\|_{L^{2}}^{2}+\int_{0}^{t}\left(\left\|\tilde{p}_{x}\right\|_{L^{2}}^{2}+\left\|\tilde{p} \tilde{p}_{x}\right\|_{L^{2}}^{2}+\varepsilon\left\|\tilde{q}_{x}\right\|_{L^{2}}^{2}\right) \mathrm{d} \tau \leq C .
$$

This completes the proof of Lemma 2.6 when $1<\gamma<2$.

\subsubsection{Proof of Lemma 2.6 when $2 \leq \gamma \leq 3$}

Step 1. Using (2.14), (2.15), and the assumptions for $\alpha$, we update (2.10) as

$$
\begin{aligned}
& \frac{\mathrm{d}}{\mathrm{d} t}\left(\widetilde{E}(\tilde{p}, \alpha)+\frac{1}{2}\|\tilde{q}\|_{L^{2}}^{2}\right)+\gamma \int_{0}^{1}\left[(\tilde{p}+\alpha)^{\gamma-2}-\alpha^{\gamma-2}\right]\left(\tilde{p}_{x}\right)^{2} \mathrm{~d} x+\gamma \alpha^{\gamma-2}\left\|\tilde{p}_{x}\right\|_{L^{2}}^{2}+\varepsilon\left\|\tilde{q}_{x}\right\|_{L^{2}}^{2} \\
\leq & C\left(\left|\alpha^{\prime}(t)\right|+\left|\beta^{\prime}(t)\right|\right) .
\end{aligned}
$$


Since $2 \leq \gamma \leq 3$, choosing $a=(\tilde{p}+\alpha) / \alpha$ in Lemma 2.3 and using Young's inequality, we deduce that

$$
\left|(\tilde{p}+\alpha)^{\gamma-2}-\alpha^{\gamma-2}\right| \leq \alpha^{\gamma-3}|\tilde{p}| \leq \frac{\alpha^{\gamma-4} \tilde{p}^{2}}{2}+\frac{\alpha^{\gamma-2}}{2}
$$

which implies

$$
(\tilde{p}+\alpha)^{\gamma-2}-\alpha^{\gamma-2} \geq-\frac{\alpha^{\gamma-4} \tilde{p}^{2}}{2}-\frac{\alpha^{\gamma-2}}{2} \geq-\frac{\underline{\alpha}^{\gamma-4} \tilde{p}^{2}}{2}-\frac{\underline{\alpha}^{\gamma-2}}{2}
$$

where the lower bound of $\alpha$ is used. Using (2.41), we update (2.40) as

$$
\begin{aligned}
& \frac{\mathrm{d}}{\mathrm{d} t}\left(\widetilde{E}(\tilde{p}, \alpha)+\frac{1}{2}\|\tilde{q}\|_{L^{2}}^{2}\right)+\frac{\gamma}{2} \underline{\alpha}^{\gamma-2}\left\|\tilde{p}_{x}\right\|_{L^{2}}^{2}-\frac{\gamma}{2} \underline{\alpha}^{\gamma-4}\left\|\tilde{p} \tilde{p}_{x}\right\|_{L^{2}}^{2}+\varepsilon\left\|\tilde{q}_{x}\right\|_{L^{2}}^{2} \\
\leq & C\left(\left|\alpha^{\prime}(t)\right|+\left|\beta^{\prime}(t)\right|\right) .
\end{aligned}
$$

Step 2. To control the term $-\frac{\gamma}{2} \underline{\alpha}^{\gamma-4} \int_{0}^{1}\left(\tilde{p} \tilde{p}_{x}\right)^{2} \mathrm{~d} x$ on the left-hand side of $(2.42)$, we multiply the first equation of $(2.13)$ by $4 \tilde{p}^{3}$, then integrate by parts to derive

$$
\frac{\mathrm{d}}{\mathrm{d} t}\|\tilde{p}\|_{L^{4}}^{4}+12\left\|\tilde{p} \tilde{p}_{x}\right\|_{L^{2}}^{2}=-12 \int_{0}^{1} \tilde{p}^{2} \tilde{p}_{x}(\tilde{p}+\alpha) \tilde{q} \mathrm{~d} x-4 \alpha^{\prime}(t) \int_{0}^{1} \tilde{p}^{3} \mathrm{~d} x .
$$

For the first term on the right-hand side of (2.43), we use Hölder's inequality to derive

$$
\begin{aligned}
& \left|-12 \int_{0}^{1} \tilde{p}^{2} \tilde{p}_{x}(\tilde{p}+\alpha) \tilde{q} \mathrm{~d} x\right| \\
\leq & 12\left(\int_{0}^{1}(\tilde{p}+\alpha)^{\gamma-2}\left(\tilde{p}_{x}\right)^{2} \mathrm{~d} x\right)^{\frac{1}{2}}\left(\int_{0}^{1}(\tilde{p}+\alpha)^{4-\gamma} \tilde{p}^{4} \tilde{q}^{2} \mathrm{~d} x\right)^{\frac{1}{2}} \\
= & 12\left(\int_{0}^{1}(\tilde{p}+\alpha)^{\gamma-2}\left(\tilde{p}_{x}\right)^{2} \mathrm{~d} x\right)^{\frac{1}{2}}\left(\int_{0}^{1}\left(\tilde{p}^{3}+\alpha \tilde{p}^{2}\right)^{4-\gamma}|\tilde{p}|^{2 \gamma-4} \tilde{q}^{2} \mathrm{~d} x\right)^{\frac{1}{2}} \\
\leq & 12\left(\int_{0}^{1}(\tilde{p}+\alpha)^{\gamma-2}\left(\tilde{p}_{x}\right)^{2} \mathrm{~d} x\right)^{\frac{1}{2}}\left\|\tilde{p}^{3}+\alpha \tilde{p}^{2}\right\|_{L^{\infty}}^{\frac{4-\gamma}{2}}\|\tilde{p}\|_{L^{\infty}}^{\gamma-2}\|\tilde{q}\|_{L^{2}} \\
\leq & C\left(\int_{0}^{1}(\tilde{p}+\alpha)^{\gamma-2}\left(\tilde{p}_{x}\right)^{2} \mathrm{~d} x\right)^{\frac{1}{2}}\left(\left\|\tilde{p}^{3}\right\|_{L^{\infty}}^{\frac{4-\gamma}{2}}+\left\|\tilde{p}^{2}\right\|_{L^{\infty}}^{\frac{4-\gamma}{2}}\right)\|\tilde{p}\|_{L^{\infty}}^{\gamma-2},
\end{aligned}
$$

where we applied the uniform estimate of $\|\tilde{q}\|_{L^{2}}$ recorded in (2.14). For the first $L^{\infty}$ estimate on the right-hand side of $(2.44)$, since $\left.\tilde{p}\right|_{x=0}=0$, using Hölder's inequality, 
we deduce

$$
\begin{aligned}
& \left|\tilde{p}^{3}(x, t)\right|=\left|3 \int_{0}^{x} \tilde{p}^{2} \tilde{p}_{x} \mathrm{~d} x\right| \leq 3 \int_{0}^{1}|\tilde{p}|^{2}\left|\tilde{p}_{x}\right| \mathrm{d} x \\
\leq & 3\left(\int_{0}^{1}|\tilde{p}|^{\gamma} \mathrm{d} x\right)^{\frac{1}{2}}\left(\int_{0}^{1}|\tilde{p}|^{4-\gamma}\left(\tilde{p}_{x}\right)^{2} \mathrm{~d} x\right)^{\frac{1}{2}} \\
\leq & C\left(\int_{0}^{1}|\tilde{p}|^{4-\gamma}\left(\tilde{p}_{x}\right)^{2} \mathrm{~d} x\right)^{\frac{1}{2}},
\end{aligned}
$$

where we applied (2.19) when deriving the last inequality. Hence, we have

$$
\left\|\tilde{p}^{3}\right\|_{L^{\infty}}^{\frac{4-\gamma}{2}} \leq C\left(\int_{0}^{1}|\tilde{p}|^{4-\gamma}\left(\tilde{p}_{x}\right)^{2} \mathrm{~d} x\right)^{\frac{4-\gamma}{4}} .
$$

Moreover, since $\left.\tilde{p}\right|_{x=0}=0$, we can show that

$$
\tilde{p}^{2}(x, t)=2 \int_{0}^{x} \tilde{p} \tilde{p}_{x} \mathrm{~d} x \leq 2\|\tilde{p}\|_{L^{2}}\left\|\tilde{p}_{x}\right\|_{L^{2}} \leq C\left\|\tilde{p}_{x}\right\|_{L^{2}}
$$

where we used (2.19) for the estimate of $\|\tilde{p}\|_{L^{2}}$. Hence, it holds that

$$
\|\tilde{p}\|_{L^{\infty}} \leq C\left\|\tilde{p}_{x}\right\|_{L^{2}}^{\frac{1}{2}}
$$

Using (2.45) and (2.46), we update (2.44) as

$$
\begin{aligned}
& \left|-12 \int_{0}^{1} \tilde{p}^{2} \tilde{p}_{x}(\tilde{p}+\alpha) \tilde{q} \mathrm{~d} x\right| \\
\leq & C\left(\int_{0}^{1}(\tilde{p}+\alpha)^{\gamma-2}\left(\tilde{p}_{x}\right)^{2} \mathrm{~d} x\right)^{\frac{1}{2}}\left[\left(\int_{0}^{1}|\tilde{p}|^{4-\gamma}\left(\tilde{p}_{x}\right)^{2} \mathrm{~d} x\right)^{\frac{4-\gamma}{4}}+\left\|\tilde{p}_{x}\right\|_{L^{2}}^{\frac{4-\gamma}{2}}\right]\left\|\tilde{p}_{x}\right\|_{L^{2}}^{\frac{\gamma-2}{2}} .
\end{aligned}
$$

When $2 \leq \gamma \leq 3$, applying Young's inequality, we can show that

$$
\begin{aligned}
& {\left[\left(\int_{0}^{1}|\tilde{p}|^{4-\gamma}\left(\tilde{p}_{x}\right)^{2} \mathrm{~d} x\right)^{\frac{4-\gamma}{4}}+\left\|\tilde{p}_{x}\right\|_{L^{2}}^{\frac{4-\gamma}{2}}\right]\left\|\tilde{p}_{x}\right\|_{L^{2}}^{\frac{\gamma-2}{2}} } \\
= & \left(\int_{0}^{1}|\tilde{p}|^{4-\gamma}\left(\tilde{p}_{x}\right)^{2} \mathrm{~d} x\right)^{\frac{4-\gamma}{4}}\left\|\tilde{p}_{x}\right\|_{L^{2}}^{\frac{\gamma-2}{2}}+\left\|\tilde{p}_{x}\right\|_{L^{2}} \\
& \left\{\begin{array}{l}
=\left(\int_{0}^{1}|\tilde{p}|^{4-\gamma}\left(\tilde{p}_{x}\right)^{2} \mathrm{~d} x\right)^{\frac{1}{2}}+\left\|\tilde{p}_{x}\right\|_{L^{2}}, \quad \text { when } \quad \gamma=2, \\
\leq\left(\int_{0}^{1}|\tilde{p}|^{4-\gamma}\left(\tilde{p}_{x}\right)^{2} \mathrm{~d} x\right)^{\frac{1}{2}}+2\left\|\tilde{p}_{x}\right\|_{L^{2}}, \quad \text { when } \quad \gamma \in(2,3],
\end{array}\right.
\end{aligned}
$$


by using which we update (2.47) as

$$
\begin{aligned}
& \left|-12 \int_{0}^{1} \tilde{p}^{2} \tilde{p}_{x}(\tilde{p}+\alpha) \tilde{q} \mathrm{~d} x\right| \\
\leq & C\left(\int_{0}^{1}(\tilde{p}+\alpha)^{\gamma-2}\left(\tilde{p}_{x}\right)^{2} \mathrm{~d} x\right)^{\frac{1}{2}}\left[\left(\int_{0}^{1}|\tilde{p}|^{4-\gamma}\left(\tilde{p}_{x}\right)^{2} \mathrm{~d} x\right)^{\frac{1}{2}}+2\left\|\tilde{p}_{x}\right\|_{L^{2}}\right] .
\end{aligned}
$$

Using Young's inequality and noticing that $\gamma \in[2,3]$, we can show that

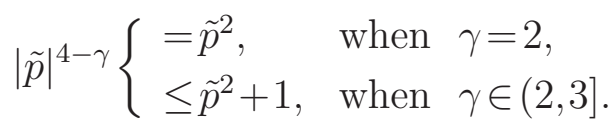

Hence, we update (2.48) as

$$
\begin{aligned}
& \left|-12 \int_{0}^{1} \tilde{p}^{2} \tilde{p}_{x}(\tilde{p}+\alpha) \tilde{q} \mathrm{~d} x\right| \\
\leq & C\left(\int_{0}^{1}(\tilde{p}+\alpha)^{\gamma-2}\left(\tilde{p}_{x}\right)^{2} \mathrm{~d} x\right)^{\frac{1}{2}}\left(\left\|\tilde{p} \tilde{p}_{x}\right\|_{L^{2}}+3\left\|\tilde{p}_{x}\right\|_{L^{2}}\right) \\
\leq & \left\|\tilde{p} \tilde{p}_{x}\right\|_{L^{2}}^{2}+C \int_{0}^{1}(\tilde{p}+\alpha)^{\gamma-2}\left(\tilde{p}_{x}\right)^{2} \mathrm{~d} x+C\left(\int_{0}^{1}(\tilde{p}+\alpha)^{\gamma-2}\left(\tilde{p}_{x}\right)^{2} \mathrm{~d} x\right)^{\frac{1}{2}}\left\|\tilde{p}_{x}\right\|_{L^{2}} .
\end{aligned}
$$

Substituting (2.48) into (2.43), we obtain

$$
\begin{aligned}
& \frac{\mathrm{d}}{\mathrm{d} t}\|\tilde{p}\|_{L^{4}}^{4}+11\left\|\tilde{p} \tilde{p}_{x}\right\|_{L^{2}}^{2} \\
\leq & C \int_{0}^{1}(\tilde{p}+\alpha)^{\gamma-2}\left(\tilde{p}_{x}\right)^{2} \mathrm{~d} x+C\left(\int_{0}^{1}(\tilde{p}+\alpha)^{\gamma-2}\left(\tilde{p}_{x}\right)^{2} \mathrm{~d} x\right)^{\frac{1}{2}}\left\|\tilde{p}_{x}\right\|_{L^{2}} \\
& +4\left|\alpha^{\prime}(t)\right| \int_{0}^{1}|\tilde{p}|^{3} \mathrm{~d} x .
\end{aligned}
$$

Next, we make a coupling of (2.42) and (2.50) to gain a non-degenerate dissipation mechanism associated with $\tilde{p}_{x}$.

Step 3. Let $M_{1}=\frac{\gamma \underline{\alpha}^{\gamma-4}}{11}$. Multiplying (2.50) by $M_{1}$, then taking the sum of the result with (2.42), we obtain

$$
\begin{aligned}
& \quad \frac{\mathrm{d}}{\mathrm{d} t}\left(\widetilde{E}(\tilde{p}, \alpha)+\frac{1}{2}\|\tilde{q}\|_{L^{2}}^{2}+M_{1}\|\tilde{p}\|_{L^{4}}^{4}\right)+\frac{\gamma}{2} \underline{\alpha}^{\gamma-2}\left\|\tilde{p}_{x}\right\|_{L^{2}}^{2}+\frac{\gamma}{2} \underline{\alpha}^{\gamma-4}\left\|\tilde{p} \tilde{p}_{x}\right\|_{L^{2}}^{2}+\varepsilon\left\|\tilde{q}_{x}\right\|_{L^{2}}^{2} \\
& \leq C\left(\left|\alpha^{\prime}(t)\right|+\left|\beta^{\prime}(t)\right|\right)+C \int_{0}^{1}(\tilde{p}+\alpha)^{\gamma-2}\left(\tilde{p}_{x}\right)^{2} \mathrm{~d} x+C\left(\int_{0}^{1}(\tilde{p}+\alpha)^{\gamma-2}\left(\tilde{p}_{x}\right)^{2} \mathrm{~d} x\right)^{\frac{1}{2}}\left\|\tilde{p}_{x}\right\|_{L^{2}} \\
& \quad+C\left|\alpha^{\prime}(t)\right| \int_{0}^{1}|\tilde{p}|^{3} \mathrm{~d} x
\end{aligned}
$$


Using Young's inequality, we estimate the last two terms on the right-hand side of $(2.51)$ as

$$
\begin{aligned}
& -C\left(\int_{0}^{1}(\tilde{p}+\alpha)^{\gamma-2}\left(\tilde{p}_{x}\right)^{2} \mathrm{~d} x\right)^{\frac{1}{2}}\left\|\tilde{p}_{x}\right\|_{L^{2}} \leq C\left(\int_{0}^{1}(\tilde{p}+\alpha)^{\gamma-2}\left(\tilde{p}_{x}\right)^{2} \mathrm{~d} x\right)+\frac{\gamma}{4} \underline{\alpha}^{\gamma-2}\left\|\tilde{p}_{x}\right\|_{L^{2}}^{2}, \\
& \text { - } C\left|\alpha^{\prime}(t)\right| \int_{0}^{1}|\tilde{p}|^{3} \mathrm{~d} x \leq C\left|\alpha^{\prime}(t)\right|\left(\|\tilde{p}\|_{L^{4}}^{4}+\|\tilde{p}\|_{L^{2}}^{2}\right) \leq C\left|\alpha^{\prime}(t)\right|\|\tilde{p}\|_{L^{4}}^{4}+C\left|\alpha^{\prime}(t)\right|,
\end{aligned}
$$

where we applied (2.19) for the uniform estimate of $\|\tilde{p}\|_{L^{2}}$. Then we update (2.51) as

$$
\begin{aligned}
& \frac{\mathrm{d}}{\mathrm{d} t}\left(\widetilde{E}(\tilde{p}, \alpha)+\frac{1}{2}\|\tilde{q}\|_{L^{2}}^{2}+M_{1}\|\tilde{p}\|_{L^{4}}^{4}\right)+\frac{\gamma}{4} \underline{\alpha}^{\gamma-2}\left\|\tilde{p}_{x}\right\|_{L^{2}}^{2}+\frac{\gamma}{2} \underline{\alpha}^{\gamma-4}\left\|\tilde{p} \tilde{p}_{x}\right\|_{L^{2}}^{2}+\varepsilon\left\|\tilde{q}_{x}\right\|_{L^{2}}^{2} \\
\leq & C\left|\alpha^{\prime}(t)\right|\|\tilde{p}\|_{L^{4}}^{4}+C\left(\left|\alpha^{\prime}(t)\right|+\left|\beta^{\prime}(t)\right|\right)+C \int_{0}^{1}(\tilde{p}+\alpha)^{\gamma-2}\left(\tilde{p}_{x}\right)^{2} \mathrm{~d} x
\end{aligned}
$$

Applying Grönwall's inequality and using the assumptions in Theorems 1.1 and (2.14), we can show that

$$
\widetilde{E}(\tilde{p}, \alpha)+\frac{1}{2}\|\tilde{q}\|_{L^{2}}^{2}+M_{1}\|\tilde{p}\|_{L^{4}}^{4} \leq C .
$$

Applying the uniform estimate of $\|\tilde{p}\|_{L^{4}}$ obtained above to (2.52), then integrating the result with respect to $t$, we finally reach the desired estimate (2.20). This competes the proof of Lemma 2.6 when $2 \leq \gamma \leq 3$.

\subsubsection{Proof of Lemma 2.6 when $3<\gamma \leq 4$}

Step 1. Since $3<\gamma \leq 4$, by choosing $a=\frac{\tilde{p}}{\alpha}$ in Lemma 2.3, we have

$$
(\tilde{p}+\alpha)^{\gamma-2} \geq \alpha^{\gamma-2}+(\gamma-2) \alpha^{\gamma-3} \tilde{p} .
$$

Note that in this case we still have (2.40), i.e.,

$$
\begin{aligned}
& \frac{\mathrm{d}}{\mathrm{d} t}\left(\widetilde{E}(\tilde{p}, \alpha)+\frac{1}{2}\|\tilde{q}\|_{L^{2}}^{2}\right)+\gamma \int_{0}^{1}(\tilde{p}+\alpha)^{\gamma-2}\left(\tilde{p}_{x}\right)^{2} \mathrm{~d} x+\varepsilon\left\|\tilde{q}_{x}\right\|_{L^{2}}^{2} \\
\leq & C\left(\left|\alpha^{\prime}(t)\right|+\left|\beta^{\prime}(t)\right|\right) .
\end{aligned}
$$

Inserting (2.54) into (2.55), we obtain

$$
\begin{aligned}
& \frac{\mathrm{d}}{\mathrm{d} t}\left(\widetilde{E}(\tilde{p}, \alpha)+\frac{1}{2}\|\tilde{q}\|_{L^{2}}^{2}\right)+\gamma \alpha^{\gamma-2}\left\|\tilde{p}_{x}\right\|_{L^{2}}^{2}+\gamma(\gamma-2) \alpha^{\gamma-3} \int_{0}^{1} \tilde{p}\left(\tilde{p}_{x}\right)^{2} \mathrm{~d} x+\varepsilon\left\|\tilde{q}_{x}\right\|_{L^{2}}^{2} \\
\leq & C\left(\left|\alpha^{\prime}(t)\right|+\left|\beta^{\prime}(t)\right|\right) .
\end{aligned}
$$


Note that since $0<\underline{\alpha} \leq \alpha \leq \bar{\alpha}$ and $3<\gamma \leq 4$, then

$$
\begin{aligned}
& \gamma \alpha^{\gamma-2}\left\|\tilde{p}_{x}\right\|_{L^{2}}^{2}+\gamma(\gamma-2) \alpha^{\gamma-3} \int_{0}^{1} \tilde{p}\left(\tilde{p}_{x}\right)^{2} \mathrm{~d} x \\
\geq & \gamma \alpha^{\gamma-2}\left\|\tilde{p}_{x}\right\|_{L^{2}}^{2}-\frac{\gamma}{2} \alpha^{\gamma-2}\left\|\tilde{p}_{x}\right\|_{L^{2}}^{2}-\frac{\gamma}{2}(\gamma-2)^{2} \alpha^{\gamma-4}\left\|\tilde{p} \tilde{p}_{x}\right\|_{L^{2}}^{2} \\
\geq & \frac{\gamma}{2} \underline{\alpha}^{\gamma-2}\left\|\tilde{p}_{x}\right\|_{L^{2}}^{2}-\frac{\gamma}{2}(\gamma-2)^{2} \max \left\{1, \underline{\alpha}^{\gamma-4}\right\}\left\|\tilde{p} \tilde{p}_{x}\right\|_{L^{2}}^{2} .
\end{aligned}
$$

Using (2.57), we update (2.56) as

$$
\begin{aligned}
& \frac{\mathrm{d}}{\mathrm{d} t}\left(\widetilde{E}(\tilde{p}, \alpha)+\frac{1}{2}\|\tilde{q}\|_{L^{2}}^{2}\right)+\frac{\gamma}{2} \underline{\alpha}^{\gamma-2}\left\|\tilde{p}_{x}\right\|_{L^{2}}^{2}-\frac{\rho_{1}}{2}\left\|\tilde{p} \tilde{p}_{x}\right\|_{L^{2}}^{2}+\varepsilon\left\|\tilde{q}_{x}\right\|_{L^{2}}^{2} \\
\leq & C\left(\left|\alpha^{\prime}(t)\right|+\left|\beta^{\prime}(t)\right|\right),
\end{aligned}
$$

where $\rho_{1}=\gamma(\gamma-2)^{2} \max \left\{1, \underline{\alpha}^{\gamma-4}\right\}$. Next, we derive a similar estimate as (2.50).

Step 2. Similar to (2.43), we can show that

$$
\begin{aligned}
& \frac{\mathrm{d}}{\mathrm{d} t}\|\tilde{p}\|_{L^{4}}^{4}+12\left\|\tilde{p} \tilde{p}_{x}\right\|_{L^{2}}^{2}=-12 \int_{0}^{1} \tilde{p}^{2} \tilde{p}_{x}(\tilde{p}+\alpha) \tilde{q} \mathrm{~d} x-4 \alpha^{\prime}(t) \int_{0}^{1} \tilde{p}^{3} \mathrm{~d} x \\
\leq & 12\left(\int_{0}^{1}(\tilde{p}+\bar{p})^{\gamma-2}\left(\tilde{p}_{x}\right)^{2}\right)^{\frac{1}{2}}\left(\int_{0}^{1}(\tilde{p}+\alpha)^{4-\gamma} \tilde{p}^{4} \tilde{q}^{2} \mathrm{~d} x\right)^{\frac{1}{2}}+4\left|\alpha^{\prime}(t)\right|\left(\|\tilde{p}\|_{L^{4}}^{4}+1\right) \\
= & 12\left(\int_{0}^{1}(\tilde{p}+\alpha)^{\gamma-2}\left(\tilde{p}_{x}\right)^{2}\right)^{\frac{1}{2}}\left(\int_{0}^{1}\left(\tilde{p}^{5}+\alpha \tilde{p}^{4}\right)^{4-\gamma}|\tilde{p}|^{4 \gamma-12} \tilde{q}^{2} \mathrm{~d} x\right)^{\frac{1}{2}}+C\left|\alpha^{\prime}(t)\right| \\
\leq & 12\left(\int_{0}^{1}(\tilde{p}+\alpha)^{\gamma-2}\left(\tilde{p}_{x}\right)^{2} \mathrm{~d} x\right)^{\frac{1}{2}}\left\|\tilde{p}^{5}+\alpha \tilde{p}^{4}\right\|_{L^{\infty}}^{\frac{4-\gamma}{2}}\|\tilde{p}\|_{L^{\infty}}^{2 \gamma-6}\|\tilde{q}\|_{L^{2}}+C\left|\alpha^{\prime}(t)\right| \\
\leq & C\left(\int_{0}^{1}(\tilde{p}+\alpha)^{\gamma-2}\left(\tilde{p}_{x}\right)^{2} \mathrm{~d} x\right)^{\frac{1}{2}}\left\|\tilde{p}^{5}+\alpha \tilde{p}^{4}\right\|_{L^{\infty}}^{\frac{4-\gamma}{2}}\left\|\tilde{p}_{x}\right\|_{L^{2}}^{\gamma-3}+C\left|\alpha^{\prime}(t)\right|,
\end{aligned}
$$

where we used (2.53) for the estimates of $\|\tilde{q}\|_{L^{2}}$ and $\|\tilde{p}\|_{L^{4}}$, and (2.46). For the $L^{\infty}$ norm of $\tilde{p}^{5}+\alpha \tilde{p}^{4}$ on the right-hand side of (2.59), we observe that

$$
\begin{aligned}
& \left|\tilde{p}^{5}(x, t)+\alpha \tilde{p}^{4}(x, t)\right| \\
= & \left|5 \int_{0}^{x} \tilde{p}^{4} \tilde{p}_{x} \mathrm{~d} x+4 \alpha \int_{0}^{x} \tilde{p}^{3} \tilde{p}_{x} \mathrm{~d} x\right| \\
\leq & 5 \int_{0}^{x}|\tilde{p}|^{3}(\tilde{p}+\alpha)\left|\tilde{p}_{x}\right| \mathrm{d} x+\alpha \int_{0}^{x}|\tilde{p}|^{3}\left|\tilde{p}_{x}\right| \mathrm{d} x \\
\leq & 5\left(\int_{0}^{1} \tilde{p}^{6}(\tilde{p}+\alpha) \mathrm{d} x\right)^{\frac{1}{2}}\left(\int_{0}^{1}(\tilde{p}+\alpha)\left(\tilde{p}_{x}\right)^{2} \mathrm{~d} x\right)^{\frac{1}{2}}+\bar{\alpha} \int_{0}^{1}|\tilde{p}|^{3}\left|\tilde{p}_{x}\right| \mathrm{d} x
\end{aligned}
$$


which implies

$$
\begin{aligned}
\left\|\tilde{p}^{5}+\alpha \tilde{p}^{4}\right\|_{L^{\infty}} & \leq 5\left\|\tilde{p}^{5}+\alpha \tilde{p}^{4}\right\|_{L^{\infty}}^{\frac{1}{2}}\|\tilde{p}\|_{L^{2}}\left(\int_{0}^{1}(\tilde{p}+\alpha)\left(\tilde{p}_{x}\right)^{2} \mathrm{~d} x\right)^{\frac{1}{2}}+\bar{\alpha} \int_{0}^{1}|\tilde{p}|^{3}\left|\tilde{p}_{x}\right| \mathrm{d} x \\
& \leq \frac{1}{2}\left\|\tilde{p}^{5}+\alpha \tilde{p}^{4}\right\|_{L^{\infty}}+C \int_{0}^{1}(\tilde{p}+\alpha)\left(\tilde{p}_{x}\right)^{2} \mathrm{~d} x+\bar{\alpha} \int_{0}^{1}|\tilde{p}|^{3}\left|\tilde{p}_{x}\right| \mathrm{d} x,
\end{aligned}
$$

where we used (2.19) for the estimate of $\|\tilde{p}\|_{L^{2}}$. For the last term on the right-hand side of (2.61), by using (2.46), we can show that

$$
\bar{\alpha} \int_{0}^{1}|\tilde{p}|^{3}\left|\tilde{p}_{x}\right| \mathrm{d} x \leq \bar{\alpha}\|\tilde{p}\|_{L^{\infty}}^{2}\left\|\tilde{p} \tilde{p}_{x}\right\|_{L^{2}} \leq C\left(\left\|\tilde{p}_{x}\right\|_{L^{2}}^{2}+\left\|\tilde{p} \tilde{p}_{x}\right\|_{L^{2}}^{2}\right) .
$$

Substituting (2.62) into (2.61), we have

$$
\left\|\tilde{p}^{5}+\alpha \tilde{p}^{4}\right\|_{L^{\infty}} \leq C \int_{0}^{1}(\tilde{p}+\alpha)\left(\tilde{p}_{x}\right)^{2} \mathrm{~d} x+C\left(\left\|\tilde{p}_{x}\right\|_{L^{2}}^{2}+\left\|\tilde{p} \tilde{p}_{x}\right\|_{L^{2}}^{2}\right) .
$$

Substituting (2.63) into (2.59), we obtain

$$
\begin{aligned}
& \frac{\mathrm{d}}{\mathrm{d} t}\|\tilde{p}\|_{L^{4}}^{4}+12\left\|\tilde{p} \tilde{p}_{x}\right\|_{L^{2}}^{2} \\
\leq & C\left(\int_{0}^{1}(\tilde{p}+\alpha)^{\gamma-2}\left(\tilde{p}_{x}\right)^{2} \mathrm{~d} x\right)^{\frac{1}{2}} \\
& \times\left(\int_{0}^{1}(\tilde{p}+\alpha)\left(\tilde{p}_{x}\right)^{2} \mathrm{~d} x+\left\|\tilde{p}_{x}\right\|_{L^{2}}^{2}+\left\|\tilde{p} \tilde{p}_{x}\right\|_{L^{2}}^{2}\right)^{\frac{4-\gamma}{2}}\left\|\tilde{p}_{x}\right\|_{L^{2}}^{\gamma-3}+C\left|\alpha^{\prime}(t)\right| .
\end{aligned}
$$

Using Young's inequality, we can show that

$$
\begin{aligned}
& \left(\int_{0}^{1}(\tilde{p}+\alpha)\left(\tilde{p}_{x}\right)^{2} \mathrm{~d} x+\left\|\tilde{p}_{x}\right\|_{L^{2}}^{2}+\left\|\tilde{p} \tilde{p}_{x}\right\|_{L^{2}}^{2}\right)^{\frac{4-\gamma}{2}}\left\|\tilde{p}_{x}\right\|_{L^{2}}^{\gamma-3} \\
\leq & \left(\int_{0}^{1}(\tilde{p}+\alpha)\left(\tilde{p}_{x}\right)^{2} \mathrm{~d} x\right)^{\frac{4-\gamma}{2}}\left\|\tilde{p}_{x}\right\|_{L^{2}}^{\gamma-3}+\left\|\tilde{p}_{x}\right\|_{L^{2}}+\left\|\tilde{p}_{x}\right\|_{L^{2}}^{4-\gamma}\left\|\tilde{p}_{x}\right\|_{L^{2}}^{\gamma-3} \\
\leq & \left(\int_{0}^{1}(\tilde{p}+\alpha)\left(\tilde{p}_{x}\right)^{2} \mathrm{~d} x\right)^{\frac{1}{2}}+\left\|\tilde{p}_{x}\right\|_{L^{2}}+\left\|\tilde{p} \tilde{p}_{x}\right\|_{L^{2}} .
\end{aligned}
$$

Using (2.65), we update (2.64) as

$$
\begin{aligned}
& \frac{\mathrm{d}}{\mathrm{d} t}\|\tilde{p}\|_{L^{4}}^{4}+12\left\|\tilde{p} \tilde{p}_{x}\right\|_{L^{2}}^{2} \\
\leq & C\left(\int_{0}^{1}(\tilde{p}+\alpha)^{\gamma-2}\left(\tilde{p}_{x}\right)^{2} \mathrm{~d} x\right)^{\frac{1}{2}}\left[\left(\int_{0}^{1}(\tilde{p}+\alpha)\left(\tilde{p}_{x}\right)^{2} \mathrm{~d} x\right)^{\frac{1}{2}}+\left\|\tilde{p}_{x}\right\|_{L^{2}}+\left\|\tilde{p} \tilde{p}_{x}\right\|_{L^{2}}\right] \\
& \quad+C\left|\alpha^{\prime}(t)\right| .
\end{aligned}
$$


Noting that

$$
\begin{aligned}
\left(\int_{0}^{1}(\tilde{p}+\alpha)\left(\tilde{p}_{x}\right)^{2} \mathrm{~d} x\right)^{\frac{1}{2}} & \leq\left(\left\|\tilde{p} \tilde{p}_{x}\right\|_{L^{2}}^{2}+(\bar{\alpha}+1)\left\|\tilde{p}_{x}\right\|_{L^{2}}^{2}\right)^{\frac{1}{2}} \\
& \leq\left\|\tilde{p} \tilde{p}_{x}\right\|_{L^{2}}+\sqrt{\bar{\alpha}+1}\left\|\tilde{p}_{x}\right\|_{L^{2}}
\end{aligned}
$$

we update (2.66) as

$$
\begin{aligned}
& \quad \frac{\mathrm{d}}{\mathrm{d} t}\|\tilde{p}\|_{L^{4}}^{4}+12\left\|\tilde{p} \tilde{p}_{x}\right\|_{L^{2}}^{2} \\
& \leq C\left(\int_{0}^{1}(\tilde{p}+\alpha)^{\gamma-2}\left(\tilde{p}_{x}\right)^{2} \mathrm{~d} x\right)^{\frac{1}{2}}\left(\left\|\tilde{p} \tilde{p}_{x}\right\|_{L^{2}}+\left\|\tilde{p}_{x}\right\|_{L^{2}}\right)+C\left|\alpha^{\prime}(t)\right| \\
& \leq\left\|\tilde{p} \tilde{p}_{x}\right\|_{L^{2}}+C \int_{0}^{1}(\tilde{p}+\alpha)^{\gamma-2}\left(\tilde{p}_{x}\right)^{2} \mathrm{~d} x+C\left(\int_{0}^{1}(\tilde{p}+\alpha)^{\gamma-2}\left(\tilde{p}_{x}\right)^{2} \mathrm{~d} x\right)^{\frac{1}{2}}\left\|\tilde{p}_{x}\right\|_{L^{2}} \\
& \quad+C\left|\alpha^{\prime}(t)\right|,
\end{aligned}
$$

which yields

$$
\begin{aligned}
& \frac{\mathrm{d}}{\mathrm{d} t}\|\tilde{p}\|_{L^{4}}^{4}+11\left\|\tilde{p} \tilde{p}_{x}\right\|_{L^{2}}^{2} \\
\leq & C \int_{0}^{1}(\tilde{p}+\alpha)^{\gamma-2}\left(\tilde{p}_{x}\right)^{2} \mathrm{~d} x+C\left(\int_{0}^{1}(\tilde{p}+\alpha)^{\gamma-2}\left(\tilde{p}_{x}\right)^{2} \mathrm{~d} x\right)^{\frac{1}{2}}\left\|\tilde{p}_{x}\right\|_{L^{2}}+C\left|\alpha^{\prime}(t)\right| .
\end{aligned}
$$

Step 3. Let $M_{2}=\frac{\rho_{1}}{11}$. Multiplying (2.68) by $M_{2}$, then adding the result to (2.58), we obtain

$$
\begin{aligned}
& \frac{\mathrm{d}}{\mathrm{d} t}\left(\widetilde{E}(\tilde{p}, \alpha)+\frac{1}{2}\|\tilde{q}\|_{L^{2}}^{2}+M_{2}\|\tilde{p}\|_{L^{4}}^{4}\right)+\frac{\gamma}{2} \underline{\alpha}^{\gamma-2}\left\|\tilde{p}_{x}\right\|_{L^{2}}^{2}+\frac{\rho_{1}}{2}\left\|\tilde{p} \tilde{p}_{x}\right\|_{L^{2}}^{2}+\varepsilon\left\|\tilde{q}_{x}\right\|_{L^{2}}^{2} \\
\leq & C \int_{0}^{1}(\tilde{p}+\alpha)^{\gamma-2}\left(\tilde{p}_{x}\right)^{2} \mathrm{~d} x+C\left(\int_{0}^{1}(\tilde{p}+\alpha)^{\gamma-2}\left(\tilde{p}_{x}\right)^{2} \mathrm{~d} x\right)^{\frac{1}{2}}\left\|\tilde{p}_{x}\right\|_{L^{2}}+C\left(\left|\alpha^{\prime}(t)\right|+\left|\beta^{\prime}(t)\right|\right) \\
\leq & \frac{\gamma}{4} \underline{\alpha}^{\gamma-2}\left\|\tilde{p}_{x}\right\|_{L^{2}}^{2}+C \int_{0}^{1}(\tilde{p}+\alpha)^{\gamma-2}\left(\tilde{p}_{x}\right)^{2} \mathrm{~d} x+C\left(\left|\alpha^{\prime}(t)\right|+\left|\beta^{\prime}(t)\right|\right)
\end{aligned}
$$

which implies

$$
\begin{aligned}
& \frac{\mathrm{d}}{\mathrm{d} t}\left(\widetilde{E}(\tilde{p}, \alpha)+\frac{1}{2}\|\tilde{q}\|_{L^{2}}^{2}+M_{2}\|\tilde{p}\|_{L^{4}}^{4}\right)+\frac{\gamma}{4} \underline{\alpha}^{\gamma-2}\left\|\tilde{p}_{x}\right\|_{L^{2}}^{2}+\frac{\rho_{1}}{2}\left\|\tilde{p} \tilde{p}_{x}\right\|_{L^{2}}^{2}+\varepsilon\left\|\tilde{q}_{x}\right\|_{L^{2}}^{2} \\
\leq & C \int_{0}^{1}(\tilde{p}+\alpha)^{\gamma-2}\left(\tilde{p}_{x}\right)^{2} \mathrm{~d} x+C\left(\left|\alpha^{\prime}(t)\right|+\left|\beta^{\prime}(t)\right|\right) .
\end{aligned}
$$

Integrating (2.70) from 0 to $t$, using (2.14) and the assumptions in Theorem 1.1, we obtain (2.20). This competes the proof of Lemma 2.6 when $3<\gamma \leq 4$. 


\subsubsection{Proof of Lemma 2.6 when $\gamma>4$}

Step 1. In this case, (2.56) is still valid. Since $\gamma>4$, using Young's inequality, we have

$$
\begin{aligned}
& \gamma \alpha^{\gamma-2}\left\|\tilde{p}_{x}\right\|_{L^{2}}^{2}+\gamma(\gamma-2) \alpha^{\gamma-3} \int_{0}^{1} \tilde{p}\left(\tilde{p}_{x}\right)^{2} \mathrm{~d} x \\
\geq & \gamma \alpha^{\gamma-2}\left\|\tilde{p}_{x}\right\|_{L^{2}}^{2}-\gamma(\gamma-2) \alpha^{\gamma-3} \int_{0}^{1}\left|\tilde{p}_{x}\right|^{\frac{2 \gamma-4}{\gamma-1}}\left|\tilde{p}_{x}\right|^{\frac{2}{\gamma-1}}|\tilde{p}| \mathrm{d} x \\
\geq & \gamma \alpha^{\gamma-2}\left\|\tilde{p}_{x}\right\|_{L^{2}}^{2}-\frac{\gamma}{2} \alpha^{\gamma-2}\left\|\tilde{p}_{x}\right\|_{L^{2}}^{2}-\frac{2^{\gamma-2} \gamma(\gamma-2)^{2 \gamma-3}}{(\gamma-1)^{\gamma-1}} \alpha^{-1} \int_{0}^{1}|\tilde{p}|^{\gamma-1}\left|\tilde{p}_{x}\right|^{2} \mathrm{~d} x \\
\geq & \frac{\gamma}{2} \underline{\alpha}^{\gamma-2}\left\|\tilde{p}_{x}\right\|_{L^{2}}^{2}-\frac{\rho_{2}}{2} \int_{0}^{1}|\tilde{p}|^{\gamma-1}\left|\tilde{p}_{x}\right|^{2} \mathrm{~d} x
\end{aligned}
$$

where

$$
\rho_{2}=\frac{2^{\gamma-1} \gamma(\gamma-2)^{2 \gamma-3}}{(\gamma-1)^{\gamma-1}} \underline{\alpha}^{-1}
$$

Similar to (2.58), we have

$$
\begin{aligned}
& \frac{\mathrm{d}}{\mathrm{d} t}\left(\widetilde{E}(\tilde{p}, \alpha)+\frac{1}{2}\|\tilde{q}\|_{L^{2}}^{2}\right)+\frac{\gamma}{2} \underline{\alpha}^{\gamma-2}\left\|\tilde{p}_{x}\right\|_{L^{2}}^{2}-\frac{\rho_{2}}{2} \int_{0}^{1}|\tilde{p}|^{\gamma-1}\left|\tilde{p}_{x}\right|^{2} \mathrm{~d} x+\varepsilon\left\|\tilde{q}_{x}\right\|_{L^{2}}^{2} \\
\leq & C\left(\left|\alpha^{\prime}(t)\right|+\left|\beta^{\prime}(t)\right|\right) .
\end{aligned}
$$

Step 2. Multiplying the first equation of $(2.13)$ by $(\gamma+1)|\tilde{p}|^{\gamma-1} \tilde{p}$, then integrating by parts with respect to $x$ over $(0,1)$, we obtain

$$
\begin{aligned}
& \frac{\mathrm{d}}{\mathrm{d} t}\left(\int_{0}^{1}|\tilde{p}|^{\gamma+1} \mathrm{~d} x\right)+\gamma(\gamma+1) \int_{0}^{1}|\tilde{p}|^{\gamma-1}\left(\tilde{p}_{x}\right)^{2} \mathrm{~d} x \\
= & -\gamma(\gamma+1) \int_{0}^{1}(\tilde{p}+\alpha) \tilde{q}|\tilde{p}|^{\gamma-1} \tilde{p}_{x} \mathrm{~d} x-(\gamma+1) \alpha^{\prime}(t) \int_{0}^{1}|\tilde{p}|^{\gamma-1} \tilde{p} \mathrm{~d} x .
\end{aligned}
$$

Since $\gamma>4$ and $\tilde{p}+\alpha>0$, we estimate the first term on the right-hand side of $(2.72)$ as

$$
\begin{aligned}
& \left.\left|\int_{0}^{1}(\tilde{p}+\alpha) \tilde{q}\right| \tilde{p}\right|^{\gamma-1} \tilde{p}_{x} \mathrm{~d} x \mid \\
\leq & \left(\int_{0}^{1}(\tilde{p}+\alpha)^{2}\left|\tilde{p}_{x}\right|^{2} \mathrm{~d} x\right)^{\frac{1}{2}}\|\tilde{q}\|_{L^{2}}\|\tilde{p}\|_{L^{\infty}}^{\gamma-1} \\
= & \left(\int_{0}^{1}(\tilde{p}+\alpha)^{2}\left|\tilde{p}_{x}\right|^{\frac{4}{\gamma-2}}\left|\tilde{p}_{x}\right|^{\frac{2 \gamma-8}{\gamma-2}} \mathrm{~d} x\right)^{\frac{1}{2}}\|\tilde{q}\|_{L^{2}}\|\tilde{p}\|_{L^{\infty}}^{\gamma-1} \\
\leq & C\left(\int_{0}^{1}(\tilde{p}+\alpha)^{\gamma-2}\left|\tilde{p}_{x}\right|^{2} \mathrm{~d} x\right)^{\frac{1}{\gamma-2}}\|\tilde{p}\|_{L^{\infty}}^{\gamma-1}\left\|\tilde{p}_{x}\right\|_{L^{2}}^{\frac{\gamma-4}{\gamma-2}},
\end{aligned}
$$


where we used the uniform estimate of $\|\tilde{q}\|_{L^{2}}$ in (2.14). For the term involving the $L^{\infty}$ norm of $\tilde{p}$ on the right-hand side of (2.73), we note that

$$
\begin{aligned}
|\tilde{p}(x, t)|^{\gamma-1} & =\int_{0}^{x}\left(\left(\tilde{p}^{2}\right)^{\frac{\gamma-1}{2}}\right)_{x} \mathrm{~d} x=(\gamma-1) \int_{0}^{x}|\tilde{p}|^{\gamma-3} \tilde{p} \tilde{p}_{x} \mathrm{~d} x \\
& \leq(\gamma-1) \int_{0}^{1}|\tilde{p}|^{\frac{\gamma}{2}}|\tilde{p}|^{\frac{\gamma}{2}-2}\left|\tilde{p}_{x}\right| \mathrm{d} x \\
& \leq(\gamma-1)\left(\int_{0}^{1}|\tilde{p}|^{\gamma} \mathrm{d} x\right)^{\frac{1}{2}}\left(\int_{0}^{1}|\tilde{p}|^{\gamma-4}\left|\tilde{p}_{x}\right|^{2} \mathrm{~d} x\right)^{\frac{1}{2}} \\
& \leq C\left(\int_{0}^{1}|\tilde{p}|^{\gamma-4}\left|\tilde{p}_{x}\right|^{2} \mathrm{~d} x\right)^{\frac{1}{2}}
\end{aligned}
$$

where we used (2.19). This implies

$$
\|\tilde{p}\|_{L^{\infty}}^{\gamma-1} \leq C\left(\int_{0}^{1}|\tilde{p}|^{\gamma-4}\left|\tilde{p}_{x}\right|^{2} \mathrm{~d} x\right)^{\frac{1}{2}} .
$$

Substituting (2.74) into (2.73), we have

$$
\begin{aligned}
& \left.\left|\int_{0}^{1}(\tilde{p}+\alpha) \tilde{q}\right| \tilde{p}\right|^{\gamma-1} \tilde{p}_{x} \mathrm{~d} x \mid \\
\leq & C\left(\int_{0}^{1}(\tilde{p}+\alpha)^{\gamma-2}\left|\tilde{p}_{x}\right|^{2} \mathrm{~d} x\right)^{\frac{1}{\gamma-2}}\left(\int_{0}^{1}|\tilde{p}|^{\gamma-4}\left|\tilde{p}_{x}\right|^{2} \mathrm{~d} x\right)^{\frac{1}{2}}\left\|\tilde{p}_{x}\right\|_{L^{2}}^{\frac{\gamma-4}{\gamma-2}} \\
\leq & C\left(\int_{0}^{1}(\tilde{p}+\alpha)^{\gamma-2}\left|\tilde{p}_{x}\right|^{2} \mathrm{~d} x\right)^{\frac{1}{\gamma-2}}\left(\int_{0}^{1}|\tilde{p}|^{\gamma-1}\left|\tilde{p}_{x}\right|^{2} \mathrm{~d} x+\left\|\tilde{p}_{x}\right\|_{L^{2}}^{2}\right)^{\frac{1}{2}}\left\|\tilde{p}_{x}\right\|_{L^{2}}^{\frac{\gamma-4}{\gamma-2}} \\
\leq & C \int_{0}^{1}(\tilde{p}+\alpha)^{\gamma-2}\left|\tilde{p}_{x}\right|^{2} \mathrm{~d} x+\delta\left(\int_{0}^{1}|\tilde{p}|^{\gamma-1}\left|\tilde{p}_{x}\right|^{2} \mathrm{~d} x+\left\|\tilde{p}_{x}\right\|_{L^{2}}^{2}\right)^{\frac{\gamma-2}{2 \gamma-6}}\left\|\tilde{p}_{x}\right\|_{L^{2}}^{\frac{\gamma-4}{\gamma-3}},
\end{aligned}
$$

where $\delta>0$ is a constant to be determined, and we used the inequality: $|\tilde{p}|^{\gamma-4} \leq$ $|\tilde{p}|^{\gamma-1}+1$ due to $\gamma>4$, and Young's inequality. For the second term on the right-hand side of (2.72), using (2.19), we can show that

$$
\left.\left|(\gamma+1) \alpha^{\prime}(t) \int_{0}^{1}\right| \tilde{p}\right|^{\gamma-1} \tilde{p} \mathrm{~d} x\left|\leq(\gamma+1)\|\tilde{p}\|_{L^{\gamma}}^{\gamma}\right| \alpha^{\prime}(t)|\leq C| \alpha^{\prime}(t) \mid .
$$

Substituting (2.75) and (2.76) into (2.72), we obtain

$$
\begin{aligned}
& \quad \frac{\mathrm{d}}{\mathrm{d} t}\left(\int_{0}^{1}|\tilde{p}|^{\gamma+1} \mathrm{~d} x\right)+\gamma(\gamma+1) \int_{0}^{1}|\tilde{p}|^{\gamma-1}\left(\tilde{p}_{x}\right)^{2} \mathrm{~d} x \\
& \leq C \int_{0}^{1}(\tilde{p}+\alpha)^{\gamma-2}\left|\tilde{p}_{x}\right|^{2} \mathrm{~d} x+\delta\left(\int_{0}^{1}|\tilde{p}|^{\gamma-1}\left|\tilde{p}_{x}\right|^{2} \mathrm{~d} x+\left\|\tilde{p}_{x}\right\|_{L^{2}}^{2}\right)^{\frac{\gamma-2}{2 \gamma-6}}\left\|\tilde{p}_{x}\right\|_{L^{2}}^{\frac{\gamma-4}{\gamma-3}} \\
& \quad+C\left|\alpha^{\prime}(t)\right| .
\end{aligned}
$$


Using Young's inequality, we can show that

$$
\begin{aligned}
& \left(\int_{0}^{1}|\tilde{p}|^{\gamma-1}\left|\tilde{p}_{x}\right|^{2} \mathrm{~d} x+\left\|\tilde{p}_{x}\right\|_{L^{2}}^{2}\right)^{\frac{\gamma-2}{2 \gamma-6}}\left\|\tilde{p}_{x}\right\|_{L^{2}}^{\frac{\gamma-4}{\gamma-3}} \\
\leq & \frac{\gamma-2}{2 \gamma-6}\left(\int_{0}^{1}|\tilde{p}|^{\gamma-1}\left|\tilde{p}_{x}\right|^{2} \mathrm{~d} x+\left\|\tilde{p}_{x}\right\|_{L^{2}}^{2}\right)+\frac{\gamma-4}{2 \gamma-6}\left\|\tilde{p}_{x}\right\|_{L^{2}}^{2} \\
\leq & \int_{0}^{1}|\tilde{p}|^{\gamma-1}\left|\tilde{p}_{x}\right|^{2} \mathrm{~d} x+\left\|\tilde{p}_{x}\right\|_{L^{2}}^{2} .
\end{aligned}
$$

Hence, we update (2.77) as

$$
\begin{aligned}
& \frac{\mathrm{d}}{\mathrm{d} t}\left(\int_{0}^{1}|\tilde{p}|^{\gamma+1} \mathrm{~d} x\right)+\gamma(\gamma+1) \int_{0}^{1}|\tilde{p}|^{\gamma-1}\left(\tilde{p}_{x}\right)^{2} \mathrm{~d} x \\
\leq & C \int_{0}^{1}(\tilde{p}+\alpha)^{\gamma-2}\left|\tilde{p}_{x}\right|^{2} \mathrm{~d} x+\delta\left(\int_{0}^{1}|\tilde{p}|^{\gamma-1}\left|\tilde{p}_{x}\right|^{2} \mathrm{~d} x+\left\|\tilde{p}_{x}\right\|_{L^{2}}^{2}\right)+C\left|\alpha^{\prime}(t)\right| .
\end{aligned}
$$

Step 3. Multiplying (2.77) by $M_{3}=\frac{\rho_{2}}{\gamma(\gamma+1)}$, then adding the result to (2.71), we have

$$
\begin{aligned}
& \frac{\mathrm{d}}{\mathrm{d} t}\left(\widetilde{E}(\tilde{p}, \alpha)+\frac{1}{2}\|\tilde{q}\|_{L^{2}}^{2}+M_{3} \int_{0}^{1}|\tilde{p}|^{\gamma+1} \mathrm{~d} x\right)+\frac{\gamma}{2} \underline{\alpha}^{\gamma-2}\left\|\tilde{p}_{x}\right\|_{L^{2}}^{2} \\
& \quad+\frac{\rho_{2}}{2} \int_{0}^{1}|\tilde{p}|^{\gamma-1}\left|\tilde{p}_{x}\right|^{2} \mathrm{~d} x+\varepsilon\left\|\tilde{q}_{x}\right\|_{L^{2}}^{2} \\
& \leq C \int_{0}^{1}(\tilde{p}+\alpha)^{\gamma-2}\left|\tilde{p}_{x}\right|^{2} \mathrm{~d} x+\delta M_{3}\left(\int_{0}^{1}|\tilde{p}|^{\gamma-1}\left|\tilde{p}_{x}\right|^{2} \mathrm{~d} x+\left\|\tilde{p}_{x}\right\|_{L^{2}}^{2}\right) \\
& \quad+C\left(\left|\alpha^{\prime}(t)\right|+\left|\beta^{\prime}(t)\right|\right) .
\end{aligned}
$$

Choosing

$$
\delta=\frac{1}{4 M_{3}} \min \left\{\gamma \underline{\alpha}^{\gamma-2}, \rho_{2}\right\}
$$

we obtain from (2.79) that

$$
\begin{aligned}
& \quad \frac{\mathrm{d}}{\mathrm{d} t}\left(\widetilde{E}(\tilde{p}, \alpha)+\frac{1}{2}\|\tilde{q}\|_{L^{2}}^{2}+M_{3} \int_{0}^{1}|\tilde{p}|^{\gamma+1} \mathrm{~d} x\right)+\frac{\gamma}{4} \underline{\alpha}^{\gamma-2}\left\|\tilde{p}_{x}\right\|_{L^{2}}^{2} \\
& \quad+\frac{\rho_{2}}{4} \int_{0}^{1}|\tilde{p}|^{\gamma-1}\left|\tilde{p}_{x}\right|^{2} \mathrm{~d} x+\varepsilon\left\|\tilde{q}_{x}\right\|_{L^{2}}^{2} \\
& \leq C \int_{0}^{1}(\tilde{p}+\alpha)^{\gamma-2}\left|\tilde{p}_{x}\right|^{2} \mathrm{~d} x+C\left(\left|\alpha^{\prime}(t)\right|+\left|\beta^{\prime}(t)\right|\right) .
\end{aligned}
$$

Integrating (2.80) with respect to time, using (2.14) and the assumptions in Theorem 1.1, we obtain (2.20). This competes the proof of Lemma 2.6 when $\gamma>4$. 


\section{$2.3 \quad H^{1}$ estimate}

Now we improve the regularity of the solution.

Lemma 2.7. Under the assumptions in Theorem 1.1, there exists a constant $C>0$, which is independent on $t$, such that

$$
\left\|\tilde{p}_{x}(t)\right\|_{L^{2}}^{2}+\left\|\tilde{q}_{x}(t)\right\|_{L^{2}}^{2}+\int_{0}^{t}\left(\left\|\tilde{p}_{x x}(\tau)\right\|_{L^{2}}^{2}+\left\|\tilde{q}_{x x}(\tau)\right\|_{L^{2}}^{2}\right) \mathrm{d} \tau \leq C .
$$

Proof. Taking $L^{2}$ inner products of the first equation of (2.13) with $-\tilde{p}_{x x}$, and the second with $-\tilde{q}_{x x}$, respectively, then adding the results, we have

$$
\begin{aligned}
& \frac{1}{2} \frac{\mathrm{d}}{\mathrm{d} t}\left(\left\|\tilde{p}_{x}\right\|_{L^{2}}^{2}+\left\|\tilde{q}_{x}\right\|_{L^{2}}^{2}\right)+\left\|\tilde{p}_{x x}\right\|_{L^{2}}^{2}+\varepsilon\left\|\tilde{q}_{x x}\right\|_{L^{2}}^{2} \\
= & \underbrace{-\int_{0}^{1}\left(\tilde{q} \tilde{p}_{x}+\tilde{p} \tilde{q}_{x}+\alpha \tilde{q}_{x}+\beta \tilde{p}_{x}\right) \tilde{p}_{x x} \mathrm{~d} x}_{\equiv R_{1}}+\underbrace{\alpha^{\prime}(t) \int_{0}^{1} \tilde{p}_{x x} \mathrm{~d} x}_{\equiv R_{2}} \\
& \underbrace{-2 \varepsilon \int_{0}^{1} \tilde{q} \tilde{q}_{x} \tilde{q}_{x x} \mathrm{~d} x}_{\equiv R_{3}}-2 \varepsilon \beta \int_{0}^{1} \tilde{q}_{x} \tilde{q}_{x x} \mathrm{~d} x-\gamma \int_{0}^{1}(\tilde{p}+\alpha)^{\gamma-1} \tilde{p}_{x} \tilde{q}_{x x} \mathrm{~d} x \\
& +\underbrace{\beta^{\prime}(t) \int_{0}^{1} \tilde{q}_{x x} \mathrm{~d} x}_{\equiv R_{5}} .
\end{aligned}
$$

Using the Cauchy-Schwarz, Sobolev and Poincaré inequalities, we can show that

$$
\begin{aligned}
R_{1} & \leq \frac{1}{4}\left\|\tilde{p}_{x x}\right\|_{L^{2}}^{2}+4\left(\|\tilde{q}\|_{L^{\infty}}^{2}\left\|\tilde{p}_{x}\right\|_{L^{2}}^{2}+\|\tilde{p}\|_{L^{\infty}}^{2}\left\|\tilde{q}_{x}\right\|_{L^{2}}^{2}+\alpha^{2}\left\|\tilde{q}_{x}\right\|_{L^{2}}^{2}+\beta^{2}\left\|\tilde{p}_{x}\right\|_{L^{2}}^{2}\right) \\
& \leq \frac{1}{4}\left\|\tilde{p}_{x x}\right\|_{L^{2}}^{2}+4\left\|\tilde{q}_{x}\right\|_{L^{2}}^{2}\left\|\tilde{p}_{x}\right\|_{L^{2}}^{2}+4 \bar{\alpha}^{2}\left\|\tilde{q}_{x}\right\|_{L^{2}}^{2}+4 \bar{\beta}^{2}\left\|\tilde{p}_{x}\right\|_{L^{2}}^{2}, \\
R_{2} & \leq \frac{1}{4}\left\|\tilde{p}_{x x}\right\|_{L^{2}}^{2}+\left|\alpha^{\prime}(t)\right|^{2}, \\
R_{3} & \leq \frac{\varepsilon}{8}\left\|\tilde{q}_{x x}\right\|_{L^{2}}^{2}+8 \varepsilon\|\tilde{q}\|_{L^{\infty}}^{2}\left\|\tilde{q}_{x}\right\|_{L^{2}}^{2} \leq \frac{\varepsilon}{8}\left\|\tilde{q}_{x x}\right\|_{L^{2}}^{2}+8 \varepsilon\left\|\tilde{q}_{x}\right\|_{L^{2}}^{2}\left\|\tilde{q}_{x}\right\|_{L^{2}}^{2}, \\
R_{4} & \leq \frac{\varepsilon}{8}\left\|\tilde{q}_{x x}\right\|_{L^{2}}^{2}+8 \varepsilon \bar{\beta}^{2}\left\|\tilde{q}_{x}\right\|_{L^{2}}^{2} .
\end{aligned}
$$

For $R_{5}$, by the Cauchy-Schwarz inequality, we have

$$
R_{5} \leq \frac{\varepsilon}{8}\left\|\tilde{q}_{x x}\right\|_{L^{2}}^{2}+\frac{2 \gamma^{2}}{\varepsilon} \int_{0}^{1}(\tilde{p}+\alpha)^{2(\gamma-1)}\left(\tilde{p}_{x}\right)^{2} \mathrm{~d} x .
$$


When $1<\gamma \leq 4$, using Sobolev and Poincaré inequalities, we can show that

$$
\begin{aligned}
& \int_{0}^{1}(\tilde{p}+\alpha)^{2(\gamma-1)}\left(\tilde{p}_{x}\right)^{2} \mathrm{~d} x \\
\leq & \|\tilde{p}+\alpha\|_{L^{\infty}}^{\gamma} \int_{0}^{1}(\tilde{p}+\alpha)^{\gamma-2}\left(\tilde{p}_{x}\right)^{2} \mathrm{~d} x \\
\leq & C\left(\|\tilde{p}\|_{L^{\infty}}^{\gamma}+\bar{\alpha}^{\gamma}\right) \int_{0}^{1}(\tilde{p}+\alpha)^{\gamma-2}\left(\tilde{p}_{x}\right)^{2} \mathrm{~d} x \\
\leq & C\left(\left\|\tilde{p}_{x}\right\|_{L^{2}}^{\frac{\gamma}{2}}\|\tilde{p}\|_{L^{2}}^{\frac{\gamma}{2}}+\bar{\alpha}^{\gamma}\right) \int_{0}^{1}(\tilde{p}+\alpha)^{\gamma-2}\left(\tilde{p}_{x}\right)^{2} \mathrm{~d} x \\
\leq & C\left(\left\|\tilde{p}_{x}\right\|_{L^{2}}^{\frac{\gamma}{2}}+1\right) \int_{0}^{1}(\tilde{p}+\alpha)^{\gamma-2}\left(\tilde{p}_{x}\right)^{2} \mathrm{~d} x \\
\leq & \left\|\tilde{p}_{x}\right\|_{L^{2}}^{2} \int_{0}^{1}(\tilde{p}+\alpha)^{\gamma-2}\left(\tilde{p}_{x}\right)^{2} \mathrm{~d} x+C \int_{0}^{1}(\tilde{p}+\alpha)^{\gamma-2}\left(\tilde{p}_{x}\right)^{2} \mathrm{~d} x,
\end{aligned}
$$

where we also used (2.19) and Young's inequality. When $\gamma>4$, by using (2.74), we have

$$
\begin{aligned}
& \int_{0}^{1}(\tilde{p}+\alpha)^{2(\gamma-1)}\left(\tilde{p}_{x}\right)^{2} \mathrm{~d} x \\
\leq & \|\tilde{p}+\alpha\|_{L^{\infty}}^{2(\gamma-1)}\left\|\tilde{p}_{x}\right\|_{L^{2}}^{2} \\
\leq & C\left(\int_{0}^{1}|\tilde{p}|^{\gamma-4}\left(\tilde{p}_{x}\right)^{2} \mathrm{~d} x+\bar{\alpha}^{2(\gamma-1)}\right)\left\|\tilde{p}_{x}\right\|_{L^{2}}^{2} \\
\leq & C\left(\int_{0}^{1} \tilde{p}^{\gamma-1}\left(\tilde{p}_{x}\right)^{2} \mathrm{~d} x+\left\|\tilde{p}_{x}\right\|_{L^{2}}^{2}\right)\left\|\tilde{p}_{x}\right\|_{L^{2}}^{2}+C\left\|\tilde{p}_{x}\right\|_{L^{2}}^{2},
\end{aligned}
$$

where we used similar arguments as in (2.75). For $R_{6}$, it is straightforward to show that

$$
R_{6} \leq \frac{\varepsilon}{8}\left\|\tilde{q}_{x x}\right\|_{L^{2}}^{2}+\frac{2}{\varepsilon}\left|\beta^{\prime}(t)\right|^{2}
$$

Substituting the estimates of $R_{i}(i=1, \cdots, 6)$ into (2.82), we have

$$
\begin{aligned}
& \quad \frac{1}{2} \frac{\mathrm{d}}{\mathrm{d} t}\left(\left\|\tilde{p}_{x}\right\|_{L^{2}}^{2}+\left\|\tilde{q}_{x}\right\|_{L^{2}}^{2}\right)+\frac{1}{2}\left\|\tilde{p}_{x x}\right\|_{L^{2}}^{2}+\frac{\varepsilon}{2}\left\|\tilde{q}_{x x}\right\|_{L^{2}}^{2} \\
& \leq C\left(\int_{0}^{1}(\tilde{p}+\alpha)^{\gamma-2}\left(\tilde{p}_{x}\right)^{2} \mathrm{~d} x+\left\|\tilde{p}_{x}\right\|_{L^{2}}^{2}+\left\|\tilde{q}_{x}\right\|_{L^{2}}^{2}\right)\left(\left\|\tilde{p}_{x}\right\|_{L^{2}}^{2}+\left\|\tilde{q}_{x}\right\|_{L^{2}}^{2}\right) \\
& \quad+C\left(\int_{0}^{1}(\tilde{p}+\alpha)^{\gamma-2}\left(\tilde{p}_{x}\right)^{2} \mathrm{~d} x+\left\|\tilde{p}_{x}\right\|_{L^{2}}^{2}+\left\|\tilde{q}_{x}\right\|_{L^{2}}^{2}+\left|\alpha^{\prime}(t)\right|^{2}+\left|\beta^{\prime}(t)\right|^{2}\right), \quad 1<\gamma \leq 4,
\end{aligned}
$$


and

$$
\begin{aligned}
& \quad \frac{1}{2} \frac{\mathrm{d}}{\mathrm{d} t}\left(\left\|\tilde{p}_{x}\right\|_{L^{2}}^{2}+\left\|\tilde{q}_{x}\right\|_{L^{2}}^{2}\right)+\frac{1}{2}\left\|\tilde{p}_{x x}\right\|_{L^{2}}^{2}+\frac{\varepsilon}{2}\left\|\tilde{q}_{x x}\right\|_{L^{2}}^{2} \\
& \leq C\left(\int_{0}^{1} \tilde{p}^{\gamma-1}\left(\tilde{p}_{x}\right)^{2} \mathrm{~d} x+\left\|\tilde{p}_{x}\right\|_{L^{2}}^{2}+\left\|\tilde{q}_{x}\right\|_{L^{2}}^{2}\right)\left(\left\|\tilde{p}_{x}\right\|_{L^{2}}^{2}+\left\|\tilde{q}_{x}\right\|_{L^{2}}^{2}\right) \\
& \quad+C\left(\left\|\tilde{p}_{x}\right\|_{L^{2}}^{2}+\left\|\tilde{q}_{x}\right\|_{L^{2}}^{2}+\left|\alpha^{\prime}(t)\right|^{2}+\left|\beta^{\prime}(t)\right|^{2}\right), \quad \gamma>4 .
\end{aligned}
$$

Applying Grönwall's inequality to (2.85) and (2.86), and using the assumptions in Theorem 1.1 and estimates established in Subsections 2.1 and 2.2, we see that the estimate (2.81) holds. This completes the proof of Lemma 2.7.

Now we show the asymptotic stability of the solution to complete the proof of Theorem 1.1.

\subsection{Global stabilization}

In what follows, we prove that $\|(\tilde{p}, \tilde{q})(t)\|_{L^{2}}^{2} \in W^{1,1}(0, \infty)$, which implies $\|(\tilde{p}, \tilde{q})(t)\|_{L^{2}}^{2} \rightarrow$ 0 as $t \rightarrow \infty$. First, from (2.14) and (2.20), we see that

$$
\left\|\tilde{p}_{x}(t)\right\|_{L^{2}}^{2}+\left\|\tilde{q}_{x}(t)\right\|_{L^{2}}^{2} \in L^{1}(0, \infty) .
$$

Then it follows from Poincaré inequality that

$$
\|\tilde{p}(t)\|_{H^{1}}^{2}+\|\tilde{q}(t)\|_{H^{1}}^{2} \in L^{1}(0, \infty) .
$$

Next, testing the two equations in (2.13) by $\tilde{p}$ and $\tilde{q}$, respectively, we have

$$
\begin{aligned}
& \frac{\mathrm{d}}{\mathrm{d} t}\left(\|\tilde{p}\|_{L^{2}}^{2}+\|\tilde{q}\|_{L^{2}}^{2}\right) \\
& =-2\left\|\tilde{p}_{x}\right\|_{L^{2}}^{2}-2 \varepsilon\left\|\tilde{q}_{x}\right\|_{L^{2}}^{2}-2 \int_{0}^{1} \tilde{p} \tilde{q} \tilde{p}_{x} \mathrm{~d} x-2 \alpha^{\prime}(t) \int_{0}^{1} \tilde{p} \mathrm{~d} x \\
& \quad+2 \alpha \int_{0}^{1} \tilde{q}_{x} \tilde{p} \mathrm{~d} x+2 \gamma \int_{0}^{1}(\tilde{p}+\alpha)^{\gamma-1} \tilde{p}_{x} \tilde{q} \mathrm{~d} x-2 \beta^{\prime}(t) \int_{0}^{1} \tilde{q} \mathrm{~d} x,
\end{aligned}
$$

from which we deduce that

$$
\begin{aligned}
& \quad\left|\frac{\mathrm{d}}{\mathrm{d} t}\left(\|\tilde{p}\|_{L^{2}}^{2}+\|\tilde{q}\|_{L^{2}}^{2}\right)\right| \\
& \leq 2\left\|\tilde{p}_{x}\right\|_{L^{2}}^{2}+2 \varepsilon\left\|\tilde{q}_{x}\right\|_{L^{2}}^{2}+\|\tilde{p}\|_{L^{\infty}}\left(\|\tilde{q}\|_{L^{2}}^{2}+\left\|\tilde{p}_{x}\right\|_{L^{2}}^{2}\right)+\left|\alpha^{\prime}(t)\right|^{2}+\|\tilde{p}\|_{L^{2}}^{2} \\
& \quad+\bar{\alpha}\left(\left\|\tilde{q}_{x}\right\|_{L^{2}}^{2}+\|\tilde{p}\|_{L^{2}}^{2}\right)+\gamma\|\tilde{p}+\alpha\|_{L^{\infty}}^{\gamma-1}\left(\left\|\tilde{p}_{x}\right\|_{L^{2}}^{2}+\|\tilde{q}\|_{L^{2}}^{2}\right) \\
& \quad+\left|\beta^{\prime}(t)\right|^{2}+\|\tilde{q}\|_{L^{2}}^{2} .
\end{aligned}
$$


Using previous energy estimates and Sobolev and Poincaré inequalities, we update (2.89) as

$$
\left|\frac{\mathrm{d}}{\mathrm{d} t}\left(\|\tilde{p}\|_{L^{2}}^{2}+\|\tilde{q}\|_{L^{2}}^{2}\right)\right| \leq C\left(\left\|\tilde{p}_{x}\right\|_{L^{2}}^{2}+\left\|\tilde{q}_{x}\right\|_{L^{2}}^{2}+\left|\alpha^{\prime}(t)\right|^{2}+\left|\beta^{\prime}(t)\right|^{2}\right) .
$$

Integrating (2.90) in time and using (2.87) and the assumptions in Theorem 1.1, we obtain

$$
\frac{\mathrm{d}}{\mathrm{d} t}\left(\|\tilde{p}(t)\|_{L^{2}}^{2}+\|\tilde{q}(t)\|_{L^{2}}^{2}\right) \in L^{1}(0, \infty)
$$

Combining (2.87) and (2.91), we obtain $\|(\tilde{p}, \tilde{q})(t)\|_{L^{2}}^{2} \in W^{1,1}(0, \infty)$, which implies $\|(\tilde{p}, \tilde{q})(t)\|_{L^{2}}^{2} \rightarrow 0$ as $t \rightarrow \infty$. Decay of the first order spatial derivatives of the perturbation can be proved in a similar fashion. We omit the details for brevity. This completes the proof of Theorem 1.1.

\section{Proof of Theorem 1.2}

In this section, we study the global dynamics of large-data solutions to the chemically non-diffusive problem. Letting $\tilde{p}=p-\alpha, \tilde{q}=q-\bar{q}$, where $\bar{q}$ denotes the spatial average of $q_{0}$, then we have a new initial-boundary value problem for $(\tilde{p}, \tilde{q})$ :

$$
\left\{\begin{array}{l}
\tilde{p}_{t}-(\tilde{p} \tilde{q})_{x}-\alpha \tilde{q}_{x}-\bar{q} \tilde{p}_{x}=\tilde{p}_{x x}-\alpha^{\prime}(t), \\
\tilde{q}_{t}-\left[(\tilde{p}+\alpha)^{\gamma}\right]_{x}=0, \\
(\tilde{p}, \tilde{q})(x, 0)=\left(p_{0}-\alpha(0), q_{0}-\bar{q}\right)(x), \\
\left.\tilde{p}\right|_{x=0,1}=0 .
\end{array}\right.
$$

Note that under the boundary conditions, it holds that

$$
\frac{\mathrm{d}}{\mathrm{d} t} \int_{0}^{1} \tilde{q}(x, t) \mathrm{d} x=0
$$

which implies

$$
\int_{0}^{1} \tilde{q}(x, t) \mathrm{d} x=\int_{0}^{1} \tilde{q}(x, 0) \mathrm{d} x=\int_{0}^{1} q_{0}(x) \mathrm{d} x-\int_{0}^{1} \bar{q} \mathrm{~d} x=0 .
$$

Hence, $\tilde{q}$ satisfies Poincaré inequality.

To prove Theorem 1.2, we first recall that the energy estimates in Lemma 2.5 and Lemma 2.6 are independent of $\varepsilon$. Moreover, it can be readily checked that the arguments in Subsections 2.1-2.2 are valid (indeed simpler) if $\beta(t)$ is a constant. Hence, by replacing $\beta(t)$ by the constant $\bar{q}$ and repeating the arguments in those two sections, one can establish the following lemma. 
Lemma 3.1. Under the conditions of Theorem 1.2, for any $\gamma \geq 2$ and $t>0$, the solution to (3.1) satisfies the following energy estimates:

$$
\begin{aligned}
& \|\tilde{p}(t)\|_{L^{2}}^{2}+\|\tilde{q}(t)\|_{L^{2}}^{2}+\int_{0}^{t}\left\|\tilde{p}_{x}(\tau)\right\|_{L^{2}}^{2} \mathrm{~d} \tau \leq C, \\
& \left\{\begin{array}{l}
\int_{0}^{t}\left\|\tilde{p} \tilde{p}_{x}(\tau)\right\|_{L^{2}}^{2} \mathrm{~d} \tau \leq C, \\
\int_{0}^{t} \int_{0}^{1}|\tilde{p}|^{\gamma-1}\left(\tilde{p}_{x}\right)^{2} \mathrm{~d} x \mathrm{~d} \tau \leq C, \text { when } \gamma \in[2,4],
\end{array}\right.
\end{aligned}
$$

where $C$ is a positive constant which is independent of $t$.

The rest of this section is largely devoted to the $H^{1}$-estimate of $(\tilde{p}, \tilde{q})$. Note that when deriving the $H^{1}$-estimate in Subsection 2.3, we used the Cauchy-Schwarz inequality by taking advantage of the positivity of $\varepsilon$, see (2.83) and (2.84). Apparently, such an approach does not work when $\varepsilon=0$. Hence, we need to develop a different method to deal with such a degeneracy. The major result of this section is recorded in the following lemma.

Lemma 3.2. Under the conditions of Theorem 1.2, for any fixed $\gamma \geq 2$ and $t>0$, it holds that

$$
\left\|\tilde{p}_{x}(t)\right\|_{L^{2}}^{2}+\left\|\tilde{q}_{x}(t)\right\|_{L^{2}}^{2}+\int_{0}^{t}\left(\left\|\tilde{p}_{x x}(\tau)\right\|_{L^{2}}^{2}+\left\|\tilde{q}_{x}(\tau)\right\|_{L^{2}}^{2}\right) \mathrm{d} \tau \leq C,
$$

where $C$ is a positive constant which is independent of $t$.

To overcome the technical barrier brought about by the lack of chemical diffusion, we take $\partial_{x}$ to the second equation of (3.1), multiply the first equation by $\gamma \alpha^{\gamma-1}$, then take difference of the resulting equations to get

$$
\begin{aligned}
\tilde{q}_{x t}= & \gamma \alpha^{\gamma-1}\left[\tilde{p}_{t}-(\tilde{p} \tilde{q})_{x}-\alpha \tilde{q}_{x}-\bar{q} \tilde{p}_{x}+\alpha^{\prime}(t)\right] \\
& +\gamma(\gamma-1)(\tilde{p}+\alpha)^{\gamma-2}\left(\tilde{p}_{x}\right)^{2}+\gamma\left[(\tilde{p}+\alpha)^{\gamma-1}-\alpha^{\gamma-1}\right] \tilde{p}_{x x} .
\end{aligned}
$$

Multiplying (3.3) by $\tilde{q}_{x}$ and integrating by parts, we can show that

$$
\begin{aligned}
& \frac{1}{2} \frac{\mathrm{d}}{\mathrm{d} t}\left\|\tilde{q}_{x}\right\|_{L^{2}}^{2}+\gamma \alpha^{\gamma}\left\|\tilde{q}_{x}\right\|_{L^{2}}^{2} \\
= & \gamma \alpha^{\gamma-1} \frac{\mathrm{d}}{\mathrm{d} t} \int_{0}^{1} \tilde{p} \tilde{q}_{x} \mathrm{~d} x-\gamma \alpha^{\gamma-1} \int_{0}^{1} \tilde{p} \tilde{q}_{x t} \mathrm{~d} x-\gamma \alpha^{\gamma-1} \int_{0}^{1} \bar{q} \tilde{p}_{x} \tilde{q}_{x} \mathrm{~d} x+\gamma \alpha^{\gamma-1} \alpha^{\prime}(t) \int_{0}^{1} \tilde{q}_{x} \mathrm{~d} x \\
& \quad-\gamma \alpha^{\gamma-1} \int_{0}^{1}(\tilde{p} \tilde{q})_{x} \tilde{q}_{x} \mathrm{~d} x+\gamma(\gamma-1) \int_{0}^{1}(\tilde{p}+\alpha)^{\gamma-2}\left(\tilde{p}_{x}\right)^{2} \tilde{q}_{x} \mathrm{~d} x \\
& +\gamma \int_{0}^{1}\left[(\tilde{p}+\alpha)^{\gamma-1}-\alpha^{\gamma-1}\right] \tilde{p}_{x x} \tilde{q}_{x} \mathrm{~d} x
\end{aligned}
$$




$$
=\frac{\mathrm{d}}{\mathrm{d} t}\left(\gamma \alpha^{\gamma-1} \int_{0}^{1} \tilde{p} \tilde{q}_{x} \mathrm{~d} x\right)-\gamma(\gamma-1) \alpha^{\gamma-2} \alpha^{\prime}(t) \int_{0}^{1} \tilde{p} \tilde{q}_{x} \mathrm{~d} x+\sum_{k=1}^{6} J_{k} .
$$

Next, we carry out energy estimates for $J_{1}-J_{6}$. The proof is divided into three subsections for the cases: $2 \leq \gamma<3,3 \leq \gamma \leq 4$, and $\gamma>4$.

\subsection{Estimates of $J_{k}$ when $2 \leq \gamma<3$}

Step 1. Note that when $2 \leq \gamma<3$, it holds that

$$
(\tilde{p}+\alpha)^{\gamma-1} \leq 2^{\gamma-2}\left(|\tilde{p}|^{\gamma-1}+\alpha^{\gamma-1}\right)
$$

Again, when $2 \leq \gamma<3$, by Young's inequality, we have $|\tilde{p}|^{\gamma-1} \leq|\tilde{p}|^{2}+1$. Then it holds that

$$
(\tilde{p}+\alpha)^{\gamma-1} \leq 2^{\gamma-2}\left(|\tilde{p}|^{2}+1+\alpha^{\gamma-1}\right)
$$

Using (3.5), integration by parts, and the assumptions for $\alpha$, we estimate $J_{1}$ as

$$
\begin{aligned}
\left|J_{1}\right| & =\gamma \alpha^{\gamma-1}\left|\int_{0}^{1} \tilde{p} \tilde{q}_{x t} \mathrm{~d} x\right|=\gamma \alpha^{\gamma-1}\left|\int_{0}^{1} \tilde{p}_{x} \tilde{q}_{t} \mathrm{~d} x\right| \\
& =\gamma^{2} \bar{\alpha}^{\gamma-1}\left|\int_{I}(\tilde{p}+\alpha)^{\gamma-1}\left(\tilde{p}_{x}\right)^{2} \mathrm{~d} x\right| \leq C\left(\left\|\tilde{p} \tilde{p}_{x}\right\|_{L^{2}}^{2}+\left\|\tilde{p}_{x}\right\|_{L^{2}}^{2}\right)
\end{aligned}
$$

where we also used the second equation of (3.1). For $J_{2}$, using the Cauchy-Schwarz inequality, we can show that

$$
\left|J_{2}\right| \leq \gamma \bar{\alpha}^{\gamma-1}|\bar{q}|\left\|\tilde{p}_{x}\right\|_{L^{2}}\left\|\tilde{q}_{x}\right\|_{L^{2}} \leq \delta\left\|\tilde{q}_{x}\right\|_{L^{2}}^{2}+C(\delta)\left\|\tilde{p}_{x}\right\|_{L^{2}}^{2}
$$

where $\delta>0$ is a constant to be determined later. Similarly, it holds that

$$
\left|J_{3}\right| \leq \gamma \bar{\alpha}^{\gamma-1}\left|\alpha^{\prime}(t)\right|\left\|\tilde{q}_{x}\right\|_{L^{1}} \leq C\left(\left|\alpha^{\prime}(t)\right|+\left|\alpha^{\prime}(t)\right|\left\|\tilde{q}_{x}\right\|_{L^{2}}^{2}\right)
$$

Using the Sobolev inequalities: $\|\tilde{p}\|_{L^{\infty}} \leq\left\|\tilde{p}_{x}\right\|_{L^{2}}$ (due to zero boundary condition) and $\|\tilde{q}\|_{L^{\infty}} \leq\left\|\tilde{q}_{x}\right\|_{L^{2}}$ (due to mean-free condition), and Lemma 3.1, we can show that

$$
\begin{aligned}
\left|J_{4}\right| & \leq \gamma \bar{\alpha}^{\gamma-1}\left(\left\|\tilde{p}_{x}\right\|_{L^{2}}\|\tilde{q}\|_{L^{\infty}}+\|\tilde{p}\|_{L^{\infty}}\left\|\tilde{q}_{x}\right\|_{L^{2}}\right)\left\|\tilde{q}_{x}\right\|_{L^{2}} \\
& \leq \delta\left\|\tilde{q}_{x}\right\|_{L^{2}}^{2}+C(\delta)\left\|\tilde{p}_{x}\right\|_{L^{2}}^{2}\left\|\tilde{q}_{x}\right\|_{L^{2}}^{2} .
\end{aligned}
$$


Since $2 \leq \gamma<3$, it holds that $(\tilde{p}+\alpha)^{\gamma-2} \leq|\tilde{p}|^{\gamma-2}+\alpha^{\gamma-2}$. Then we estimate $J_{5}$ as

$$
\begin{aligned}
\left|J_{5}\right| & =\gamma(\gamma-1)\left|\int_{0}^{1}(\tilde{p}+\alpha)^{\gamma-2}\left(\tilde{p}_{x}\right)^{2} \tilde{q}_{x} \mathrm{~d} x\right| \\
& \leq C\left(\int_{0}^{1}|\tilde{p}|^{\gamma-2}\left(\tilde{p}_{x}\right)^{2}\left|\tilde{q}_{x}\right| \mathrm{d} x+\int_{0}^{1}\left(\tilde{p}_{x}\right)^{2}\left|\tilde{q}_{x}\right| \mathrm{d} x\right) \\
& \leq C\left(\int_{0}^{1}|\tilde{p}|^{2(\gamma-2)}\left(\tilde{p}_{x}\right)^{2} \mathrm{~d} x\right)^{\frac{1}{2}}\left\|\tilde{p}_{x}\right\|_{L^{\infty}}\left\|\tilde{q}_{x}\right\|_{L^{2}}+C\left\|\tilde{p}_{x}\right\|_{L^{\infty}}\left\|\tilde{p}_{x}\right\|_{L^{2}}\left\|\tilde{q}_{x}\right\|_{L^{2}} .
\end{aligned}
$$

Since $2 \leq \gamma<3$, then $0 \leq 2(\gamma-2)<2$. It follows from Young's inequality that

$$
|\tilde{p}|^{2(\gamma-2)} \leq|\tilde{p}|^{2}+1
$$

Since $\left.\tilde{p}\right|_{x=0,1}=0$, then using Poincaré's inequality, we can show that

$$
\left\|\tilde{p}_{x}\right\|_{L^{2}}^{2}=\int_{0}^{1} \tilde{p}_{x} \tilde{p}_{x} \mathrm{~d} x=-\int_{0}^{1} \tilde{p} \tilde{p}_{x x} \mathrm{~d} x \leq\|\tilde{p}\|_{L^{2}}\left\|\tilde{p}_{x x}\right\|_{L^{2}} \leq C\left\|\tilde{p}_{x}\right\|_{L^{2}}\left\|\tilde{p}_{x x}\right\|_{L^{2}},
$$

which implies

$$
\left\|\tilde{p}_{x}\right\|_{L^{2}} \leq\left\|\tilde{p}_{x x}\right\|_{L^{2}}
$$

For $\left\|\tilde{p}_{x}\right\|_{L^{\infty}}$, using Gagliardo-Nirenberg inequality and (3.12), we have

$$
\left\|\tilde{p}_{x}\right\|_{L^{\infty}} \leq C\left(\left\|\tilde{p}_{x}\right\|_{L^{2}}^{\frac{1}{2}}\left\|\tilde{p}_{x x}\right\|_{L^{2}}^{\frac{1}{2}}+\left\|\tilde{p}_{x}\right\|_{L^{2}}\right) \leq C\left\|\tilde{p}_{x}\right\|_{L^{2}}^{\frac{1}{2}}\left\|\tilde{p}_{x x}\right\|_{L^{2}}^{\frac{1}{2}} .
$$

Using (3.11) and (3.13), we update (3.14) as

$$
\begin{aligned}
\left|J_{5}\right| & \leq C\left(\left\|\tilde{p} \tilde{p}_{x}\right\|_{L^{2}}^{2}+\left\|\tilde{p}_{x}\right\|_{L^{2}}^{2}\right)^{\frac{1}{2}}\left\|\tilde{p}_{x}\right\|_{L^{2}}^{\frac{1}{2}}\left\|\tilde{p}_{x x}\right\|_{L^{2}}^{\frac{1}{2}}\left\|\tilde{q}_{x}\right\|_{L^{2}} \\
& \leq \delta\left\|\tilde{p}_{x x}\right\|_{L^{2}}^{2}+C(\delta)\left(\left\|\tilde{p} \tilde{p}_{x}\right\|_{L^{2}}^{2}+\left\|\tilde{p}_{x}\right\|_{L^{2}}^{2}\right)^{\frac{2}{3}}\left\|\tilde{p}_{x}\right\|_{L^{2}}^{\frac{2}{3}}\left\|\tilde{q}_{x}\right\|_{L^{2}}^{\frac{4}{3}} \\
& \leq \delta\left\|\tilde{p}_{x x}\right\|_{L^{2}}^{2}+C(\delta)\left[\left(\left\|\tilde{p} \tilde{p}_{x}\right\|_{L^{2}}^{2}+\left\|\tilde{p}_{x}\right\|_{L^{2}}^{2}\right)\left\|\tilde{q}_{x}\right\|_{L^{2}}^{2}+\left\|\tilde{p}_{x}\right\|_{L^{2}}^{2}\right] .
\end{aligned}
$$

Note that by Taylor's theorem, $(\tilde{p}+\alpha)^{\gamma-1}-\alpha^{\gamma-1}=(\gamma-1) w^{\gamma-2} \tilde{p}$, where $w$ is between $\tilde{p}+\alpha$ and $\alpha$. Since $\tilde{p}+\alpha \geq 0$ and $\alpha>0$, we must have $0 \leq w \leq|\tilde{p}|+\alpha$. Moreover, since $2 \leq \gamma<3$, it holds that $w^{\gamma-2} \leq(|\tilde{p}|+\alpha)^{\gamma-2}$. Hence, we have

$$
\left|(\tilde{p}+\alpha)^{\gamma-1}-\alpha^{\gamma-1}\right| \leq(\gamma-1)(|\tilde{p}|+\alpha)^{\gamma-2}|\tilde{p}| \leq(\gamma-1)\left(|\tilde{p}|^{\gamma-2}+\alpha^{\gamma-2}\right)|\tilde{p}| .
$$


Using (3.15), we can estimate $J_{6}$ as

$$
\begin{aligned}
\left|J_{6}\right| & =\gamma \int_{0}^{1}\left|(\tilde{p}+\alpha)^{\gamma-1}-\alpha^{\gamma-1}\right|\left|\tilde{p}_{x x}\right|\left|\tilde{q}_{x}\right| \mathrm{d} x \\
& \leq \gamma(\gamma-1) \int_{0}^{1}\left(|\tilde{p}|^{\gamma-2}+\bar{\alpha}^{\gamma-2}\right)\left|\tilde{p} \| \tilde{p}_{x x}\right|\left|\tilde{q}_{x}\right| \mathrm{d} x \\
& \leq \gamma(\gamma-1)\|\tilde{p}\|_{L^{\infty}}^{\gamma-1}\left\|\tilde{p}_{x x}\right\|_{L^{2}}\left\|\tilde{q}_{x}\right\|_{L^{2}}+\gamma(\gamma-1) \bar{\alpha}^{\gamma-2}\|\tilde{p}\|_{L^{\infty}}\left\|\tilde{p}_{x x}\right\|_{L^{2}}\left\|\tilde{q}_{x}\right\|_{L^{2}} \\
& \leq C\left(\left\|\tilde{p}_{x}\right\|_{L^{2}}^{\frac{\gamma-1}{2}}+\left\|\tilde{p}_{x}\right\|_{L^{2}}^{\frac{1}{2}}\right)\left\|\tilde{p}_{x x}\right\|_{L^{2}}\left\|\tilde{q}_{x}\right\|_{L^{2}},
\end{aligned}
$$

where we used the interpolation inequality:

$$
\|\tilde{p}\|_{L^{\infty}} \leq \sqrt{2}\|\tilde{p}\|_{L^{2}}^{\frac{1}{2}}\left\|\tilde{p}_{x}\right\|_{L^{2}}^{\frac{1}{2}}
$$

and Lemma 3.1. Using Young's inequality, we can show that

$$
\begin{aligned}
& C\left(\left\|\tilde{p}_{x}\right\|_{L^{2}}^{\frac{\gamma-1}{2}}+\left\|\tilde{p}_{x}\right\|_{L^{2}}^{\frac{1}{2}}\right)\left\|\tilde{p}_{x x}\right\|_{L^{2}}\left\|\tilde{q}_{x}\right\|_{L^{2}} \\
\leq & \delta\left\|\tilde{p}_{x x}\right\|_{L^{2}}^{2}+C(\delta)\left(\left\|\tilde{p}_{x}\right\|_{L^{2}}^{\gamma-1}\left\|\tilde{q}_{x}\right\|_{L^{2}}^{2}+\left\|\tilde{p}_{x}\right\|_{L^{2}}\left\|\tilde{q}_{x}\right\|_{L^{2}}^{2}\right),
\end{aligned}
$$

where the two terms on the right-hand side can be estimated as

$$
\begin{aligned}
& C(\delta)\left\|\tilde{p}_{x}\right\|_{L^{2}}^{\gamma-1}\left\|\tilde{q}_{x}\right\|_{L^{2}}^{2} \leq\left(C(\delta)\left\|\tilde{p}_{x}\right\|_{L^{2}}^{2}+\delta\right)\left\|\tilde{q}_{x}\right\|_{L^{2}}^{2}, \\
& \left\|\tilde{p}_{x}\right\|_{L^{2}}\left\|\tilde{q}_{x}\right\|_{L^{2}}^{2} \leq C(\delta)\left\|\tilde{p}_{x}\right\|_{L^{2}}^{2}\left\|\tilde{q}_{x}\right\|_{L^{2}}^{2}+\delta\left\|\tilde{q}_{x}\right\|_{L^{2}}^{2} .
\end{aligned}
$$

Hence, we update (3.16) as

$$
\left|J_{6}\right| \leq \delta\left\|\tilde{p}_{x x}\right\|_{L^{2}}^{2}+2 \delta\left\|q_{x}\right\|_{L^{2}}^{2}+C(\delta)\left\|\tilde{p}_{x}\right\|_{L^{2}}^{2}\left\|\tilde{q}_{x}\right\|_{L^{2}}^{2} .
$$

Substituting the estimates (3.6)-(3.9), (3.14) and (3.17) into (3.4), we have

$$
\begin{aligned}
& \frac{\mathrm{d}}{\mathrm{d} t}\left(\frac{1}{2}\left\|\tilde{q}_{x}\right\|_{L^{2}}^{2}-\gamma \alpha^{\gamma-1} \int_{0}^{1} \tilde{p} \tilde{q}_{x} \mathrm{~d} x\right)+\gamma \alpha^{\gamma}\left\|\tilde{q}_{x}\right\|_{L^{2}}^{2} \\
& \leq-\gamma(\gamma-1) \alpha^{\gamma-2} \alpha^{\prime}(t) \int_{0}^{1} \tilde{p} \tilde{q}_{x} \mathrm{~d} x+2 \delta\left\|\tilde{p}_{x x}\right\|_{L^{2}}^{2}+4 \delta\left\|\tilde{q}_{x}\right\|_{L^{2}}^{2} \\
& \quad+C(\delta)\left(\left\|\tilde{p} \tilde{p}_{x}\right\|_{L^{2}}^{2}+\left\|\tilde{p}_{x}\right\|_{L^{2}}^{2}+\left|\alpha^{\prime}(t)\right|\right)\left\|\tilde{q}_{x}\right\|_{L^{2}}^{2} \\
& \quad+C(\delta)\left(\left\|\tilde{p} \tilde{p}_{x}\right\|_{L^{2}}^{2}+\left\|\tilde{p}_{x}\right\|_{L^{2}}^{2}+\left|\alpha^{\prime}(t)\right|\right) .
\end{aligned}
$$

Using Lemma 3.1, we estimate the first term on the right-hand side of (3.18) as

$$
\begin{aligned}
& \left|-\gamma(\gamma-1) \alpha^{\gamma-2} \alpha^{\prime}(t) \int_{0}^{1} \tilde{p} \tilde{q}_{x} \mathrm{~d} x\right| \\
\leq & \gamma(\gamma-1) \bar{\alpha}^{\gamma-2}\left|\alpha^{\prime}(t)\right|\left(\|\tilde{p}\|_{L^{2}}^{2}+\left\|\tilde{q}_{x}\right\|_{L^{2}}^{2}\right) \\
\leq & C\left|\alpha^{\prime}(t)\right|\left(1+\left\|\tilde{q}_{x}\right\|_{L^{2}}^{2}\right) .
\end{aligned}
$$


So we update (3.18) as

$$
\begin{aligned}
& \quad \frac{\mathrm{d}}{\mathrm{d} t}\left(\frac{1}{2}\left\|\tilde{q}_{x}\right\|_{L^{2}}^{2}-\gamma \alpha^{\gamma-1} \int_{0}^{1} \tilde{p} \tilde{q}_{x} \mathrm{~d} x\right)+\gamma \underline{\alpha}^{\gamma}\left\|\tilde{q}_{x}\right\|_{L^{2}}^{2} \\
& \leq 2 \delta\left\|\tilde{p}_{x x}\right\|_{L^{2}}^{2}+4 \delta\left\|\tilde{q}_{x}\right\|_{L^{2}}^{2}+C(\delta)\left(\left\|\tilde{p} \tilde{p}_{x}\right\|_{L^{2}}^{2}+\left\|\tilde{p}_{x}\right\|_{L^{2}}^{2}+\left|\alpha^{\prime}(t)\right|\right)\left\|\tilde{q}_{x}\right\|_{L^{2}}^{2} \\
& \quad+C(\delta)\left(\left\|\tilde{p} \tilde{p}_{x}\right\|_{L^{2}}^{2}+\left\|\tilde{p}_{x}\right\|_{L^{2}}^{2}+\left|\alpha^{\prime}(t)\right|\right),
\end{aligned}
$$

where we used the lower bound of $\alpha$. Next, we turn to the estimate of $\tilde{p}_{x}$.

Step 2. Taking $L^{2}$ inner product of the first equation of $(3.1)$ with $-\tilde{p}_{x x}$, we can show that

$$
\begin{aligned}
& \frac{1}{2} \frac{\mathrm{d}}{\mathrm{d} t}\left\|\tilde{p}_{x}\right\|_{L^{2}}^{2}+\left\|\tilde{p}_{x x}\right\|_{L^{2}}^{2} \\
= & -\int_{0}^{1}(\tilde{p} \tilde{q})_{x} \tilde{p}_{x x} \mathrm{~d} x-\alpha \int_{0}^{1} \tilde{q}_{x} \tilde{p}_{x x} \mathrm{~d} x-\bar{q} \int_{0}^{1} \tilde{p}_{x} \tilde{p}_{x x} \mathrm{~d} x+\alpha^{\prime}(t) \int_{0}^{1} \tilde{p}_{x x} \mathrm{~d} x \\
\leq & \frac{1}{2}\left\|\tilde{p}_{x x}\right\|_{L^{2}}^{2}+2\left(\left\|\tilde{p}_{x} \tilde{q}+\tilde{p} \tilde{q}_{x}\right\|_{L^{2}}^{2}+\alpha^{2}\left\|\tilde{q}_{x}\right\|_{L^{2}}^{2}+\bar{q}^{2}\left\|\tilde{p}_{x}\right\|_{L^{2}}^{2}+\left|\alpha^{\prime}(t)\right|^{2}\right) \\
\leq & \frac{1}{2}\left\|\tilde{p}_{x x}\right\|_{L^{2}}^{2}+2 \bar{\alpha}^{2}\left\|\tilde{q}_{x}\right\|_{L^{2}}^{2}+C\left(\left\|\tilde{p}_{x}\right\|_{L^{2}}^{2}\left\|\tilde{q}_{x}\right\|_{L^{2}}^{2}+\left\|\tilde{p}_{x}\right\|_{L^{2}}^{2}+\left|\alpha^{\prime}(t)\right|^{2}\right),
\end{aligned}
$$

where we used similar arguments as those in deriving (3.9). After rearranging terms, we have

$$
\frac{1}{2} \frac{\mathrm{d}}{\mathrm{d} t}\left\|\tilde{p}_{x}\right\|_{L^{2}}^{2}+\frac{1}{2}\left\|\tilde{p}_{x x}\right\|_{L^{2}}^{2} \leq 2 \bar{\alpha}^{2}\left\|\tilde{q}_{x}\right\|_{L^{2}}^{2}+C\left(\left\|\tilde{p}_{x}\right\|_{L^{2}}^{2}\left\|\tilde{q}_{x}\right\|_{L^{2}}^{2}+\left\|\tilde{p}_{x}\right\|_{L^{2}}^{2}+\left|\alpha^{\prime}(t)\right|^{2}\right),
$$

Let $\Theta_{1} \equiv \frac{3 \bar{\alpha}^{2}}{\gamma \underline{\gamma} \gamma}$. Taking the sum of $(3.21)$ and $(3.19) \times \Theta_{1}$, we obtain

$$
\begin{aligned}
& \quad \frac{\mathrm{d}}{\mathrm{d} t}\left(\frac{1}{2}\left\|\tilde{p}_{x}\right\|_{L^{2}}^{2}+\frac{\Theta_{1}}{2}\left\|\tilde{q}_{x}\right\|_{L^{2}}^{2}-\Theta_{1} \gamma \alpha^{\gamma-1} \int_{0}^{1} \tilde{p} \tilde{q}_{x} \mathrm{~d} x\right)+\frac{1}{2}\left\|\tilde{p}_{x x}\right\|_{L^{2}}^{2}+\bar{\alpha}^{2}\left\|\tilde{q}_{x}\right\|_{L^{2}}^{2} \\
& \leq 2 \Theta_{1} \delta\left\|\tilde{p}_{x x}\right\|_{L^{2}}^{2}+4 \Theta_{1} \delta\left\|\tilde{q}_{x}\right\|_{L^{2}}^{2}+C(\delta)\left(\left\|\tilde{p} \tilde{p}_{x}\right\|_{L^{2}}^{2}+\left\|\tilde{p}_{x}\right\|_{L^{2}}^{2}+\left|\alpha^{\prime}(t)\right|\right)\left\|\tilde{q}_{x}\right\|_{L^{2}}^{2} \\
& \quad+C(\delta)\left(\left\|\tilde{p} \tilde{p}_{x}\right\|_{L^{2}}^{2}+\left\|\tilde{p}_{x}\right\|_{L^{2}}^{2}+\left|\alpha^{\prime}(t)\right|+\left|\alpha^{\prime}(t)\right|^{2}\right) .
\end{aligned}
$$

Choosing $\delta=\min \left\{\left(8 \Theta_{1}\right)^{-1}, \bar{\alpha}^{2}\left(8 \Theta_{1}\right)^{-1}\right\}$, we update (3.23) as

$$
\begin{aligned}
& \quad \frac{\mathrm{d}}{\mathrm{d} t}\left(\frac{1}{2}\left\|\tilde{p}_{x}\right\|_{L^{2}}^{2}+\frac{\Theta_{1}}{2}\left\|\tilde{q}_{x}\right\|_{L^{2}}^{2}-\Theta_{1} \gamma \alpha^{\gamma-1} \int_{0}^{1} \tilde{p} \tilde{q}_{x} \mathrm{~d} x\right)+\frac{1}{4}\left\|\tilde{p}_{x x}\right\|_{L^{2}}^{2}+\frac{\bar{\alpha}^{2}}{2}\left\|\tilde{q}_{x}\right\|_{L^{2}}^{2} \\
& \leq C\left(\left\|\tilde{p} \tilde{p}_{x}\right\|_{L^{2}}^{2}+\left\|\tilde{p}_{x}\right\|_{L^{2}}^{2}+\left|\alpha^{\prime}(t)\right|\right)\left\|\tilde{q}_{x}\right\|_{L^{2}}^{2} \\
& \quad+C\left(\left\|\tilde{p} \tilde{p}_{x}\right\|_{L^{2}}^{2}+\left\|\tilde{p}_{x}\right\|_{L^{2}}^{2}+\left|\alpha^{\prime}(t)\right|+\left|\alpha^{\prime}(t)\right|^{2}\right) .
\end{aligned}
$$


Note that the quantity inside the temporal derivative in (3.23) may not be positive. In order to apply Grönwall's inequality, we make coupling of (3.23) with the entropy estimate (2.8).

Step 3. First, we observe that the quantity inside the temporal derivative in (3.23) satisfies

$$
\frac{\Theta_{1}}{2}\left\|\tilde{q}_{x}\right\|_{L^{2}}^{2}-\Theta_{1} \gamma \alpha^{\gamma-1} \int_{0}^{1} \tilde{p} \tilde{q}_{x} \mathrm{~d} x \geq \frac{\Theta_{1}}{4}\left\|\tilde{q}_{x}\right\|_{L^{2}}^{2}-\Theta_{1} \gamma^{2} \bar{\alpha}^{2(\gamma-1)}\|\tilde{p}\|_{L^{2}}^{2} .
$$

Next, we recall the entropy estimate (2.8) which, for (3.1), reads

$$
\frac{\mathrm{d}}{\mathrm{d} t}\left(\widetilde{E}(\tilde{p}, \alpha)+\frac{1}{2}\|\tilde{q}\|_{L^{2}}^{2}\right)+\gamma \int_{0}^{1}(\tilde{p}+\alpha)^{\gamma-2}\left(\tilde{p}_{x}\right)^{2} \mathrm{~d} x=-\gamma \alpha^{\gamma-2} \alpha^{\prime}(t) \int_{0}^{1} \tilde{p} \mathrm{~d} x
$$

where $\widetilde{E}(\tilde{p}, \alpha)$ is defined in $(2.15)$. Note that according to $(2.17)$, it holds that

$$
\widetilde{E}(\tilde{p}, \alpha) \geq \frac{\gamma}{2(\gamma-1)} \underline{\alpha}^{\gamma-2}\|\tilde{p}\|_{L^{2}}^{2}
$$

Let $\Theta_{2} \equiv \frac{4(\gamma-1) \Theta_{1} \bar{\alpha}^{2(\gamma-1)}}{\underline{\underline{\alpha}}^{\gamma-2}}$. Multiplying (3.25) by $\Theta_{2}$, then adding the result to (3.23), we obtain

$$
\begin{aligned}
& \quad \frac{\mathrm{d}}{\mathrm{d} t} G_{1}(t)+H_{1}(t) \\
& \leq C\left(\left\|\tilde{p} \tilde{p}_{x}\right\|_{L^{2}}^{2}+\left\|\tilde{p}_{x}\right\|_{L^{2}}^{2}+\left|\alpha^{\prime}(t)\right|\right)\left\|\tilde{q}_{x}\right\|_{L^{2}}^{2} \\
& \quad+C\left(\left\|\tilde{p} \tilde{p}_{x}\right\|_{L^{2}}^{2}+\left\|\tilde{p}_{x}\right\|_{L^{2}}^{2}+\left|\alpha^{\prime}(t)\right|+\left|\alpha^{\prime}(t)\right|^{2}\right)-\Theta_{2} \gamma \alpha^{\gamma-2} \alpha^{\prime}(t) \int_{0}^{1} \tilde{p} \mathrm{~d} x,
\end{aligned}
$$

where

$$
\begin{aligned}
G_{1}(t) & =\frac{1}{2}\left\|\tilde{p}_{x}\right\|_{L^{2}}^{2}+\frac{\Theta_{1}}{2}\left\|\tilde{q}_{x}\right\|_{L^{2}}^{2}-\Theta_{1} \gamma \alpha^{\gamma-1} \int_{0}^{1} \tilde{p} \tilde{q}_{x} \mathrm{~d} x+\Theta_{2} \widetilde{E}(\tilde{p}, \alpha)+\frac{\Theta_{2}}{2}\|\tilde{q}\|_{L^{2}}^{2}, \\
H_{1}(t) & =\frac{1}{4}\left\|\tilde{p}_{x x}\right\|_{L^{2}}^{2}+\frac{\bar{\alpha}^{2}}{2}\left\|\tilde{q}_{x}\right\|_{L^{2}}^{2}+\Theta_{2} \gamma \int_{0}^{1}(\tilde{p}+\alpha)^{\gamma-2}\left(\tilde{p}_{x}\right)^{2} \mathrm{~d} x .
\end{aligned}
$$

Note that according to (3.24), (3.26) and the definition of $\Theta_{2}$, it holds that

$$
G_{1}(t) \geq \frac{1}{2}\left\|\tilde{p}_{x}\right\|_{L^{2}}^{2}+\frac{\Theta_{1}}{4}\left\|\tilde{q}_{x}\right\|_{L^{2}}^{2}+\Theta_{1} \gamma^{2} \bar{\alpha}^{2(\gamma-1)}\|\tilde{p}\|_{L^{2}}^{2}+\frac{\Theta_{2}}{2}\|\tilde{q}\|_{L^{2}}^{2} .
$$

Hence, we update (3.27) as

$$
\begin{aligned}
\frac{\mathrm{d}}{\mathrm{d} t} G_{1}(t)+H_{1}(t) \leq C & \left(\left\|\tilde{p} \tilde{p}_{x}\right\|_{L^{2}}^{2}+\left\|\tilde{p}_{x}\right\|_{L^{2}}^{2}+\left|\alpha^{\prime}(t)\right|\right) G_{1}(t) \\
& +C\left(\left\|\tilde{p} \tilde{p}_{x}\right\|_{L^{2}}^{2}+\left\|\tilde{p}_{x}\right\|_{L^{2}}^{2}+\left|\alpha^{\prime}(t)\right|+\left|\alpha^{\prime}(t)\right|^{2}\right)
\end{aligned}
$$


where we applied the Cauchy-Schwarz and Poincaré's inequalities to the last term on the right-hand side. Applying Grönwall's inequality to (3.29) and using Lemma 3.1 and the assumptions for $\alpha$, we can show that

$$
G_{1}(t)+\int_{0}^{t} H_{1}(\tau) \mathrm{d} \tau \leq C,
$$

where the constant $C>0$ is independent of $t$. By (3.28) and definition of $H_{1}(t)$, (3.30) yields (3.2). This completes the proof of Lemma 3.2 when $2 \leq \gamma<3$.

\subsection{Estimates of $J_{k}$ when $3 \leq \gamma \leq 4$}

Step 1. Similar to (3.6), we can show that

$$
\begin{aligned}
\left|J_{1}\right| & =\gamma^{2} \alpha^{\gamma-1}\left|\int_{0}^{1}(\tilde{p}+\alpha)^{\gamma-1}\left(\tilde{p}_{x}\right)^{2} \mathrm{~d} x\right| \\
& \leq \gamma^{2} \bar{\alpha}^{\gamma-1} 2^{\gamma-2}\left|\int_{0}^{1}\left(|\tilde{p}|^{\gamma-1}+\bar{\alpha}^{\gamma-1}\right)\left(\tilde{p}_{x}\right)^{2} \mathrm{~d} x\right| \\
& \leq C\left(\|\tilde{p}\|_{L^{\infty}}^{\gamma-1}+1\right)\left\|\tilde{p}_{x}\right\|_{L^{2}}^{2} .
\end{aligned}
$$

For the $L^{\infty}$ norm on the right-hand side of (3.31), using $\left.\tilde{p}\right|_{x=0}=0$, we can show that

$$
\begin{aligned}
& |\tilde{p}|^{(\gamma-1)}(x, t)=\left(\tilde{p}^{2}\right)^{\frac{\gamma-1}{2}}(x, t)=\int_{0}^{x}\left[\left(\tilde{p}^{2}\right)^{\frac{\gamma-1}{2}}\right]_{y} \mathrm{~d} y \\
= & (\gamma-1) \int_{0}^{x}|\tilde{p}|^{\gamma-3} \tilde{p} \tilde{p}_{y} \mathrm{~d} y \\
\leq & (\gamma-1)\left(\int_{0}^{1}|\tilde{p}|^{2(\gamma-3)}\left(\tilde{p}_{x}\right)^{2} \mathrm{~d} x\right)^{\frac{1}{2}}\|\tilde{p}\|_{L^{2}} \\
\leq & C\left(\int_{0}^{1}|\tilde{p}|^{2(\gamma-3)}\left(\tilde{p}_{x}\right)^{2} \mathrm{~d} x\right)^{\frac{1}{2}},
\end{aligned}
$$

where we used Lemma 3.1 when deriving the last inequality. Since $x \in(0,1)$ is arbitrary, the estimate (3.32) implies

$$
\|\tilde{p}(\cdot, t)\|_{L^{\infty}}^{\gamma-1} \leq C\left(\int_{0}^{1}|\tilde{p}|^{2(\gamma-3)}\left(\tilde{p}_{x}\right)^{2} \mathrm{~d} x\right)^{\frac{1}{2}} .
$$

Note that since $3 \leq \gamma \leq 4$, it follows from Young's inequality that

$$
|\tilde{p}|^{2(\gamma-3)} \leq|\tilde{p}|^{2}+1
$$


by which we update (3.33) as

$$
\|\tilde{p}(\cdot, t)\|_{L^{\infty}}^{\gamma-1} \leq C\left(\left\|\tilde{p} \tilde{p}_{x}\right\|_{L^{2}}^{2}+\left\|\tilde{p}_{x}\right\|_{L^{2}}^{2}\right)^{\frac{1}{2}}
$$

Using (3.35) and the Cauchy-Schwarz inequality, we update (3.31) as

$$
\left|J_{1}\right| \leq C\left(\left\|\tilde{p} \tilde{p}_{x}\right\|_{L^{2}}^{2}+\left\|\tilde{p}_{x}\right\|_{L^{2}}^{2}\right)\left\|\tilde{p}_{x}\right\|_{L^{2}}^{2}+C\left\|\tilde{p}_{x}\right\|_{L^{2}}^{2} .
$$

The estimates of $J_{2}, J_{3}$ and $J_{4}$ are identical to (3.7), (3.8) and (3.9), respectively. Next, we re-estimate $J_{5}$ and $J_{6}$.

Step 2. For $J_{5}$, since $3 \leq \gamma \leq 4$, the function $z^{\gamma-2}$ is convex on $[0, \infty)$. This implies

$$
(\tilde{p}+\alpha)^{\gamma-2} \leq 2^{\gamma-3}\left(|\tilde{p}|^{\gamma-2}+\alpha^{\gamma-2}\right)
$$

Then we can show that

$$
\begin{aligned}
\left|J_{5}\right| & =\gamma(\gamma-1)\left|\int_{0}^{1}(\tilde{p}+\alpha)^{\gamma-2}\left(\tilde{p}_{x}\right)^{2} \tilde{q}_{x} \mathrm{~d} x\right| \\
& \leq C\left(\int_{0}^{1}|\tilde{p}|^{\gamma-2}\left(\tilde{p}_{x}\right)^{2}\left|\tilde{q}_{x}\right| \mathrm{d} x+\int_{0}^{1}\left(\tilde{p}_{x}\right)^{2}\left|\tilde{q}_{x}\right| \mathrm{d} x\right) .
\end{aligned}
$$

For the first term on the right-hand side of (3.37), using (3.34), we can show that

$$
\begin{aligned}
& C \int_{0}^{1}|\tilde{p}|^{\gamma-2}\left(\tilde{p}_{x}\right)^{2}\left|\tilde{q}_{x}\right| \mathrm{d} x \\
\leq & C\|\tilde{p}\|_{L^{\infty}}\left\|\tilde{p}_{x}\right\|_{L^{\infty}} \int_{0}^{1}|\tilde{p}|^{\gamma-3}\left|\tilde{p}_{x}\right|\left|\tilde{q}_{x}\right| \mathrm{d} x \\
\leq & C\|\tilde{p}\|_{L^{\infty}}\left\|\tilde{p}_{x}\right\|_{L^{\infty}}\left(\int_{0}^{1}|\tilde{p}|^{2(\gamma-3)}\left|\tilde{p}_{x}\right|^{2} \mathrm{~d} x\right)^{\frac{1}{2}}\left\|\tilde{q}_{x}\right\|_{L^{2}} \\
\leq & C\|\tilde{p}\|_{L^{\infty}}\left\|\tilde{p}_{x}\right\|_{L^{\infty}}\left(\left\|\tilde{p} \tilde{p}_{x}\right\|_{L^{2}}^{2}+\left\|\tilde{p}_{x}\right\|_{L^{2}}^{2}\right)^{\frac{1}{2}}\left\|\tilde{q}_{x}\right\|_{L^{2}} .
\end{aligned}
$$

Since $\left.\tilde{p}\right|_{x=0}=0$, we have

$$
\|\tilde{p}\|_{L^{\infty}} \leq \sqrt{2}\|\tilde{p}\|_{L^{2}}^{\frac{1}{2}}\left\|\tilde{p}_{x}\right\|_{L^{2}}^{\frac{1}{2}},
$$

which together with Lemma 3.1 and the estimate (3.13), implies

$$
\|\tilde{p}\|_{L^{\infty}}\left\|\tilde{p}_{x}\right\|_{L^{\infty}} \leq C\left\|\tilde{p}_{x}\right\|_{L^{2}}\left\|\tilde{p}_{x x}\right\|_{L^{2}}^{\frac{1}{2}} .
$$


Then we update (3.38) as

$$
\begin{aligned}
& C \int_{0}^{1}|\tilde{p}|^{\gamma-2}\left(\tilde{p}_{x}\right)^{2}\left|\tilde{q}_{x}\right| \mathrm{d} x \\
\leq & C\left\|\tilde{p}_{x}\right\|_{L^{2}}\left\|\tilde{p}_{x x}\right\|_{L^{2}}^{\frac{1}{2}}\left(\left\|\tilde{p} \tilde{p}_{x}\right\|_{L^{2}}^{2}+\left\|\tilde{p}_{x}\right\|_{L^{2}}^{2}\right)^{\frac{1}{2}}\left\|\tilde{q}_{x}\right\|_{L^{2}} \\
\leq & \frac{\delta}{2}\left\|\tilde{p}_{x x}\right\|_{L^{2}}^{2}+C(\delta)\left\|\tilde{p}_{x}\right\|_{L^{2}}^{\frac{4}{3}}\left(\left\|\tilde{p} \tilde{p}_{x}\right\|_{L^{2}}^{2}+\left\|\tilde{p}_{x}\right\|_{L^{2}}^{2}\right)^{\frac{2}{3}}\left\|\tilde{q}_{x}\right\|_{L^{2}}^{\frac{4}{3}} \\
\leq & \frac{\delta}{2}\left\|\tilde{p}_{x x}\right\|_{L^{2}}^{2}+C(\delta)\left(\left\|\tilde{p} \tilde{p}_{x}\right\|_{L^{2}}^{2}+\left\|\tilde{p}_{x}\right\|_{L^{2}}^{2}+\left\|\tilde{p}_{x}\right\|_{L^{2}}^{2}\left\|\tilde{q}_{x}\right\|_{L^{2}}^{2}\right),
\end{aligned}
$$

where we used Young's inequality. For the second term on the right-hand side of (3.37), we can show that

$$
\begin{aligned}
C \int_{0}^{1}\left(\tilde{p}_{x}\right)^{2}\left|\tilde{q}_{x}\right| \mathrm{d} x & \leq C\left\|\tilde{p}_{x}\right\|_{L^{\infty}}\left\|\tilde{p}_{x}\right\|_{L^{2}}\left\|\tilde{q}_{x}\right\|_{L^{2}} \\
& \leq C\left\|\tilde{p}_{x}\right\|_{L^{2}}^{\frac{3}{2}}\left\|\tilde{p}_{x x}\right\|_{L^{2}}^{\frac{1}{2}}\left\|\tilde{q}_{x}\right\|_{L^{2}} \\
& \leq \frac{\delta}{2}\left\|\tilde{p}_{x x}\right\|_{L^{2}}^{2}+C(\delta)\left\|\tilde{p}_{x}\right\|_{L^{2}}^{2}\left\|\tilde{q}_{x}\right\|_{L^{2}}^{\frac{4}{3}} \\
& \leq \frac{\delta}{2}\left\|\tilde{p}_{x x}\right\|_{L^{2}}^{2}+C(\delta)\left(\left\|\tilde{p}_{x}\right\|_{L^{2}}^{2}+\left\|\tilde{p}_{x}\right\|_{L^{2}}^{2}\left\|\tilde{q}_{x}\right\|_{L^{2}}^{2}\right)
\end{aligned}
$$

Using (3.39) and (3.40), we update (3.37) as

$$
\left|J_{5}\right| \leq \delta\left\|\tilde{p}_{x x}\right\|_{L^{2}}^{2}+C(\delta)\left(\left\|\tilde{p} \tilde{p}_{x}\right\|_{L^{2}}^{2}+\left\|\tilde{p}_{x}\right\|_{L^{2}}^{2}+\left\|\tilde{p}_{x}\right\|_{L^{2}}^{2}\left\|\tilde{q}_{x}\right\|_{L^{2}}^{2}\right) .
$$

For $J_{6}$, similar to $(3.16)$, we can show that

$$
\left|J_{6}\right| \leq \gamma(\gamma-1)\|\tilde{p}\|_{L^{\infty}}^{\gamma-1}\left\|\tilde{p}_{x x}\right\|_{L^{2}}\left\|\tilde{q}_{x}\right\|_{L^{2}}+\gamma(\gamma-1) \bar{\alpha}^{\gamma-2}\|\tilde{p}\|_{L^{\infty}}\left\|\tilde{p}_{x x}\right\|_{L^{2}}\left\|\tilde{q}_{x}\right\|_{L^{2}}
$$

For the first term on the right-hand side of (3.42), using (3.35), we can show that

$$
\begin{aligned}
& \gamma(\gamma-1)\|\tilde{p}\|_{L^{\infty}}^{\gamma-1}\left\|\tilde{p}_{x x}\right\|_{L^{2}}\left\|\tilde{q}_{x}\right\|_{L^{2}} \\
\leq & C\left(\left\|\tilde{p} \tilde{p}_{x}\right\|_{L^{2}}^{2}+\left\|\tilde{p}_{x}\right\|_{L^{2}}^{2}\right)^{\frac{1}{2}}\left\|\tilde{p}_{x x}\right\|_{L^{2}}\left\|\tilde{q}_{x}\right\|_{L^{2}} \\
\leq & \frac{\delta}{2}\left\|\tilde{p}_{x x}\right\|_{L^{2}}^{2}+C(\delta)\left(\left\|\tilde{p} \tilde{p}_{x}\right\|_{L^{2}}^{2}+\left\|\tilde{p}_{x}\right\|_{L^{2}}^{2}\right)\left\|\tilde{q}_{x}\right\|_{L^{2}}^{2} .
\end{aligned}
$$

For the second term on the right-hand side of (3.42), we can show that

$$
\begin{aligned}
& \gamma(\gamma-1) \bar{\alpha}^{\gamma-2}\|\tilde{p}\|_{L^{\infty}}\left\|\tilde{p}_{x x}\right\|_{L^{2}}\left\|\tilde{q}_{x}\right\|_{L^{2}} \\
\leq & C\left\|\tilde{p}_{x}\right\|_{L^{2}}\left\|\tilde{p}_{x x}\right\|\left\|_{L^{2}}\right\| \tilde{q}_{x} \|_{L^{2}} \\
\leq & \frac{\delta}{2}\left\|\tilde{p}_{x x}\right\|_{L^{2}}^{2}+C(\delta)\left\|\tilde{p}_{x}\right\|_{L^{2}}^{2}\left\|\tilde{q}_{x}\right\|_{L^{2}}^{2} .
\end{aligned}
$$


Using (3.43) and (3.44), we update (3.42) as

$$
\left|J_{6}\right| \leq \delta\left\|\tilde{p}_{x x}\right\|_{L^{2}}^{2}+C(\delta)\left(\left\|\tilde{p} \tilde{p}_{x}\right\|_{L^{2}}^{2}+\left\|\tilde{p}_{x}\right\|_{L^{2}}^{2}\right)\left\|\tilde{q}_{x}\right\|_{L^{2}}^{2} .
$$

Using the estimates for $J_{k}$, we can get an inequality that is similar to (3.19):

$$
\begin{aligned}
& \quad \frac{\mathrm{d}}{\mathrm{d} t}\left(\frac{1}{2}\left\|\tilde{q}_{x}\right\|_{L^{2}}^{2}-\gamma \alpha^{\gamma-1} \int_{0}^{1} \tilde{p} \tilde{q}_{x} \mathrm{~d} x\right)+\gamma \underline{\alpha}^{\gamma}\left\|\tilde{q}_{x}\right\|_{L^{2}}^{2} \\
& \leq 2 \delta\left\|\tilde{p}_{x x}\right\|_{L^{2}}^{2}+2 \delta\left\|\tilde{q}_{x}\right\|_{L^{2}}^{2}+C(\delta)\left(\left\|\tilde{p} \tilde{p}_{x}\right\|_{L^{2}}^{2}+\left\|\tilde{p}_{x}\right\|_{L^{2}}^{2}+\left|\alpha^{\prime}(t)\right|\right)\left(\left\|\tilde{q}_{x}\right\|_{L^{2}}^{2}+\left\|\tilde{p}_{x}\right\|_{L^{2}}^{2}\right) \\
& \quad+C(\delta)\left(\left\|\tilde{p} \tilde{p}_{x}\right\|_{L^{2}}^{2}+\left\|\tilde{p}_{x}\right\|_{L^{2}}^{2}+\left|\alpha^{\prime}(t)\right|\right) .
\end{aligned}
$$

By repeating the arguments in Step 2 and Step 3 in Subsection 3.1, we can establish (3.2). This completes the proof of Lemma 3.2 when $3 \leq \gamma \leq 4$.

\subsection{Estimates of $J_{k}$ when $\gamma>4$}

Similar to Subsection 3.2 , we only need to estimate $J_{1}, J_{5}$ and $J_{6}$. For $J_{1}$, it follows from (3.31) that

$$
\left|J_{1}\right| \leq C\left(\int_{0}^{1}|\tilde{p}|^{\gamma-1}\left(\tilde{p}_{x}\right)^{2} \mathrm{~d} x+\left\|\tilde{p}_{x}\right\|_{L^{2}}^{2}\right)
$$

Note that the right-hand side of (3.47) is uniformly integrable with respect to time, thanks to Lemma 3.1. For $J_{5}$, recalling (3.37), we have

$$
\begin{aligned}
\left|J_{5}\right| & \leq C\left(\int_{0}^{1}|\tilde{p}|^{\gamma-2}\left(\tilde{p}_{x}\right)^{2}\left|\tilde{q}_{x}\right| \mathrm{d} x+\int_{0}^{1}\left(\tilde{p}_{x}\right)^{2}\left|\tilde{q}_{x}\right| \mathrm{d} x\right) \\
& \leq C\left(\int_{0}^{1}|\tilde{p}|^{\gamma-1}\left(\tilde{p}_{x}\right)^{2}\left|\tilde{q}_{x}\right| \mathrm{d} x+\int_{0}^{1}\left(\tilde{p}_{x}\right)^{2}\left|\tilde{q}_{x}\right| \mathrm{d} x\right),
\end{aligned}
$$

where we used Young's inequality: $|\tilde{p}|^{\gamma-2} \leq|\tilde{p}|^{\gamma-1}+1$. For the first term on the right-hand side of (3.48), we can show that

$$
\begin{aligned}
C \int_{0}^{1}|\tilde{p}|^{\gamma-1}\left(\tilde{p}_{x}\right)^{2}\left|\tilde{q}_{x}\right| \mathrm{d} x & \leq C\left\|\tilde{p}_{x}\right\|_{L^{\infty}}\left(\int_{0}^{1}|\tilde{p}|^{2(\gamma-1)}\left(\tilde{p}_{x}\right)^{2} \mathrm{~d} x\right)^{\frac{1}{2}}\left\|\tilde{q}_{x}\right\|_{L^{2}} \\
& \leq C\left\|\tilde{p}_{x}\right\|_{L^{\infty}}\|\tilde{p}\|_{L^{\infty}}^{\frac{\gamma-1}{2}}\left(\int_{0}^{1}|\tilde{p}|^{\gamma-1}\left(\tilde{p}_{x}\right)^{2} \mathrm{~d} x\right)^{\frac{1}{2}}\left\|\tilde{q}_{x}\right\|_{L^{2}}
\end{aligned}
$$

Recalling (2.74), we have

$$
\|\tilde{p}\|_{L^{\infty}}^{\gamma-1} \leq C\left(\int_{0}^{1}|\tilde{p}|^{\gamma-4}\left|\tilde{p}_{x}\right|^{2} \mathrm{~d} x\right)^{\frac{1}{2}} \leq C\left(\int_{0}^{1}|\tilde{p}|^{\gamma-1}\left|\tilde{p}_{x}\right|^{2} \mathrm{~d} x+\left\|\tilde{p}_{x}\right\|_{L^{2}}^{2}\right)^{\frac{1}{2}},
$$


where we used Young's inequality due to $\gamma>4$. Using (3.50), we update (3.49) as

$$
\begin{aligned}
& C \int_{0}^{1}|\tilde{p}|^{\gamma-1}\left(\tilde{p}_{x}\right)^{2}\left|\tilde{q}_{x}\right| \mathrm{d} x \\
\leq & C\left\|\tilde{p}_{x}\right\|_{L^{2}}^{\frac{1}{2}}\left\|\tilde{p}_{x x}\right\|_{L^{2}}^{\frac{1}{2}}\left(\int_{0}^{1}|\tilde{p}|^{\gamma-1}\left|\tilde{p}_{x}\right|^{2} \mathrm{~d} x+\left\|\tilde{p}_{x}\right\|_{L^{2}}^{2}\right)^{\frac{1}{4}}\left(\int_{0}^{1}|\tilde{p}|^{\gamma-1}\left(\tilde{p}_{x}\right)^{2} \mathrm{~d} x\right)^{\frac{1}{2}}\left\|\tilde{q}_{x}\right\|_{L^{2}} \\
\leq & \frac{\delta}{2}\left\|\tilde{p}_{x x}\right\|_{L^{2}}^{2}+C(\delta)\left\|\tilde{p}_{x}\right\|_{L^{2}}^{\frac{2}{3}}\left(\int_{0}^{1}|\tilde{p}|^{\gamma-1}\left|\tilde{p}_{x}\right|^{2} \mathrm{~d} x+\left\|\tilde{p}_{x}\right\|_{L^{2}}^{2}\right)^{\frac{1}{3}}\left(\int_{0}^{1}|\tilde{p}|^{\gamma-1}\left(\tilde{p}_{x}\right)^{2} \mathrm{~d} x\right)^{\frac{2}{3}}\left\|\tilde{q}_{x}\right\|_{L^{2}}^{\frac{4}{3}} \\
\leq & \frac{\delta}{2}\left\|\tilde{p}_{x x}\right\|_{L^{2}}^{2}+C(\delta)\left(\left\|\tilde{p}_{x}\right\|_{L^{2}}^{2}+\left\|\tilde{q}_{x}\right\|_{L^{2}}^{2}\right)\left(\int_{0}^{1}|\tilde{p}|^{\gamma-1}\left|\tilde{p}_{x}\right|^{2} \mathrm{~d} x+\left\|\tilde{p}_{x}\right\|_{L^{2}}^{2}\right)
\end{aligned}
$$

where we used Young's inequality at various places. The estimate of the second term on the right-hand side of (3.48) is identical to (3.40). Hence, we obtain

$$
\begin{gathered}
\left|J_{5}\right| \leq \delta\left\|\tilde{p}_{x x}\right\|_{L^{2}}^{2}+C(\delta)\left(\left\|\tilde{p}_{x}\right\|_{L^{2}}^{2}+\left\|\tilde{q}_{x}\right\|_{L^{2}}^{2}\right)\left(\int_{0}^{1}|\tilde{p}|^{\gamma-1}\left|\tilde{p}_{x}\right|^{2} \mathrm{~d} x+\left\|\tilde{p}_{x}\right\|_{L^{2}}^{2}\right) \\
+C(\delta)\left\|\tilde{p}_{x}\right\|_{L^{2}}^{2} .
\end{gathered}
$$

Lastly, similar to (3.42)-(3.44), using (3.50), we can show that

$$
\left|J_{6}\right| \leq \delta\left\|\tilde{p}_{x x}\right\|_{L^{2}}^{2}+\left(\int_{0}^{1}|\tilde{p}|^{\gamma-1}\left|\tilde{p}_{x}\right|^{2} \mathrm{~d} x+\left\|\tilde{p}_{x}\right\|_{L^{2}}^{2}\right)\left\|\tilde{q}_{x}\right\|_{L^{2}}^{2} .
$$

Following the arguments in Subsection 3.1 and using Lemma 3.1, we can establish (3.2). This completes the proof of Lemma 3.2 when $\gamma>4$.

Lemma 3.1 and Lemma 3.2 provide the desired energy estimates for the solution to (3.1), as stated in Theorem 1.2. Long-time behavior of the solution can be established via the same method in Subsection 2.4 for the diffusive problem. We omit the technical details to simplify the presentation. This completes the proof of Theorem 1.2.

\section{Proof of Theorem 1.3}

In this section, we give a sketched proof of Theorem 1.3. Recall the IBVP:

$$
\begin{cases}p_{t}-(p q)_{x}=p_{x x}, & x \in(0,1), \quad t>0, \\ q_{t}-\left(p^{\gamma}+\varepsilon q^{2}\right)_{x}=\varepsilon q_{x x}, & x \in(0,1), \quad t>0, \\ (p, q)(x, 0)=\left(p_{0}, q_{0}\right)(x), & x \in(0,1), \\ \left.p\right|_{x=0,1}=0,\left.\quad q\right|_{x=0,1}=0, & t>0, \quad \text { when } \varepsilon>0, \\ \left.p\right|_{x=0,1}=0, & t>0, \quad \text { when } \varepsilon=0 .\end{cases}
$$




\subsection{Energy estimates}

Step 1. For any $\varepsilon \geq 0$, testing the first equation of (4.1) with $\frac{\gamma}{\gamma-1} p^{\gamma-1}$ and the second equation with $q$, and adding the results, we can show that

$$
\frac{\mathrm{d}}{\mathrm{d} t}\left(\frac{1}{\gamma-1} \int_{0}^{1} p^{\gamma} \mathrm{d} x+\frac{1}{2}\|q\|_{L^{2}}^{2}\right)+\gamma \int_{0}^{1} p^{\gamma-2}\left(p_{x}\right)^{2} \mathrm{~d} x+\varepsilon\left\|q_{x}\right\|_{L^{2}}^{2}=0,
$$

which implies

$$
\int_{0}^{1} p^{\gamma} \mathrm{d} x+\|q\|_{L^{2}}^{2}+\int_{0}^{t}\left(\int_{0}^{1} p^{\gamma-2}\left(p_{x}\right)^{2} \mathrm{~d} x+\varepsilon\left\|q_{x}\right\|_{L^{2}}^{2}\right) \mathrm{d} \tau \leq C,
$$

where $C$ is independent on $t$ and $\varepsilon$. Multiplying the first equation of (4.1) by $p$, integrating by parts, and applying interpolation and the Cauchy-Schwarz inequalities, we can show that

$$
\begin{aligned}
& \frac{1}{2} \frac{\mathrm{d}}{\mathrm{d} t}\|p\|_{L^{2}}^{2}+\left\|p_{x}\right\|_{L^{2}}^{2}=-\int_{0}^{1} p q p_{x} \mathrm{~d} x \\
\leq & \frac{1}{4}\left\|p_{x}\right\|_{L^{2}}^{2}+\|p\|_{L^{\infty}}^{2}\|q\|_{L^{2}}^{2} \\
\leq & \frac{1}{4}\left\|p_{x}\right\|_{L^{2}}^{2}+2\|p\|_{L^{2}}\left\|p_{x}\right\|_{L^{2}}\|q\|_{L^{2}}^{2} \\
\leq & \frac{1}{2}\left\|p_{x}\right\|_{L^{2}}^{2}+4\|p\|_{L^{2}}^{2}\|q\|_{L^{2}}^{4},
\end{aligned}
$$

which, together with (4.2), implies

$$
\frac{\mathrm{d}}{\mathrm{d} t}\|p\|_{L^{2}}^{2}+\left\|p_{x}\right\|_{L^{2}}^{2} \leq C\|p\|_{L^{2}}^{2}
$$

Applying Grönwall's inequality to (4.3), we infer that

$$
\|p(t)\|_{L^{2}}^{2}+\int_{0}^{t}\left\|p_{x}(\tau)\right\|_{L^{2}}^{2} \mathrm{~d} \tau \leq C(t)
$$

where $C(t)$ is increasing with respect to $t$, but is independent of $\varepsilon$. Moreover, using similar arguments in previous sections, we can show that

$$
\begin{cases}\int_{0}^{t}\left\|p p_{x}\right\|_{L^{2}}^{2} \mathrm{~d} \tau \leq C(t), & \text { when } 2 \leq \gamma \leq 4 \\ \int_{0}^{t} \int_{0}^{1} p^{\gamma-1}\left(p_{x}\right)^{2} \mathrm{~d} x \mathrm{~d} \tau \leq C(t), & \text { when } \gamma>4\end{cases}
$$


where the constant $C(t)$ is increasing with respect to $t$, but is independent of $\varepsilon$.

Step 2. Note that since $\gamma \geq 2$ and $\left.p\right|_{x=0,1}=0$, then it holds that $\left.\left(p^{\gamma}\right)_{x}\right|_{x=0,1}=0$. Also note that when $\varepsilon>0$, since $\left.q\right|_{x=0,1}=0$, it holds that $\left.q_{t}\right|_{x=0,1}=0$. Hence, the second equation of (4.1) implies $\left.q_{x x}\right|_{x=0,1}=0$. For any $\varepsilon \geq 0$, taking $L^{2}$ inner product of the first equation of (4.1) with $-p_{x x}$, differentiating the second one with respect to $x$, then taking $L^{2}$ inner product of the resulting equation with $q_{x}$, and using the boundary conditions, we can show that

$$
\begin{aligned}
& \frac{1}{2} \frac{\mathrm{d}}{\mathrm{d} t}\left(\left\|p_{x}\right\|_{L^{2}}^{2}+\left\|q_{x}\right\|_{L^{2}}^{2}\right)+\left\|p_{x x}\right\|_{L^{2}}^{2}+\varepsilon\left\|q_{x x}\right\|_{L^{2}} \\
= & \underbrace{-\int_{0}^{1}\left(p_{x} q+p q_{x}\right) p_{x x} \mathrm{~d} x-2 \varepsilon \int_{0}^{1} q q_{x} q_{x x} \mathrm{~d} x}_{\equiv I_{1}} \\
& +\underbrace{\gamma(\gamma-1) \int_{0}^{1} p^{\gamma-2}\left(p_{x}\right)^{2} q_{x} \mathrm{~d} x}_{\equiv I_{2}}+\underbrace{\gamma \int_{0}^{1} p^{\gamma-1} p_{x x} q_{x} \mathrm{~d} x}_{\equiv I_{3}},
\end{aligned}
$$

where integration by parts is applied to obtain $I_{2}$ when $\varepsilon>0$. Using interpolation, Sobolev, and Young inequalities and (4.2), we can show that

$$
\begin{aligned}
\left|I_{1}\right| & \leq\left(\left\|p_{x}\right\|_{L^{\infty}}\|q\|_{L^{2}}+\|p\|_{L^{\infty}}\left\|q_{x}\right\|_{L^{2}}\right)\left\|p_{x x}\right\|_{L^{2}} \\
& \leq C\left\|p_{x}\right\|_{L^{2}}^{\frac{1}{2}}\left\|p_{x x}\right\|_{L^{2}}^{\frac{3}{2}}+C\left\|p_{x}\right\|_{L^{2}}\left\|q_{x}\right\|_{L^{2}}\left\|p_{x x}\right\|_{L^{2}} \\
& \leq \frac{1}{6}\left\|p_{x x}\right\|_{L^{2}}^{2}+C\left(\left\|p_{x}\right\|_{L^{2}}^{2}+\left\|p_{x}\right\|_{L^{2}}^{2}\left\|q_{x}\right\|_{L^{2}}^{2}\right),
\end{aligned}
$$

and

$$
\left|I_{2}\right| \leq \frac{\varepsilon}{2}\left\|q_{x x}\right\|_{L^{2}}^{2}+2 \varepsilon\|q\|_{L^{2}}^{2}\left\|q_{x}\right\|_{L^{2}}^{2} \leq \frac{\varepsilon}{2}\left\|q_{x x}\right\|_{L^{2}}^{2}+C \varepsilon\left\|q_{x}\right\|_{L^{2}}^{2}
$$

Step 3. When $2 \leq \gamma \leq 3$, since $p^{2(\gamma-2)} \leq p^{2}+1$, we can show that

$$
\begin{aligned}
\left|I_{3}\right| & \leq \gamma(\gamma-1)\left(\int_{0}^{1} p^{2(\gamma-2)}\left(p_{x}\right)^{2} \mathrm{~d} x\right)^{\frac{1}{2}}\left\|p_{x}\right\|_{L^{\infty}}\left\|q_{x}\right\|_{L^{2}} \\
& \leq C\left(\left\|p p_{x}\right\|_{L^{2}}^{2}+\left\|p_{x}\right\|_{L^{2}}^{2}\right)^{\frac{1}{2}}\left\|p_{x}\right\|_{L^{2}}^{\frac{1}{2}}\left\|p_{x x}\right\|_{L^{2}}^{\frac{1}{2}}\left\|q_{x}\right\|_{L^{2}} \\
& \leq \frac{1}{6}\left\|p_{x x}\right\|_{L^{2}}^{2}+C\left[\left(\left\|p p_{x}\right\|_{L^{2}}^{2}+\left\|p_{x}\right\|_{L^{2}}^{2}\right)\left\|q_{x}\right\|_{L^{2}}^{2}+\left\|p_{x}\right\|_{L^{2}}^{2}\right]
\end{aligned}
$$


and by (4.4), we have

$$
\begin{aligned}
\left|I_{4}\right| & \leq \gamma\|p\|_{L^{\infty}}^{\gamma-1}\left\|p_{x x}\right\|_{L^{2}}\left\|q_{x}\right\|_{L^{2}} \\
& \leq C\|p\|_{L^{2}}^{\frac{\gamma-1}{2}}\left\|p_{x}\right\|_{L^{2}}^{\frac{\gamma-1}{2}}\left\|p_{x x}\right\|_{L^{2}}\left\|q_{x}\right\|_{L^{2}} \\
& \leq C(t)\left\|p_{x}\right\|^{\frac{\gamma-1}{2}}\left\|p_{x x}\right\|_{L^{2}}\left\|q_{x}\right\|_{L^{2}} \\
& \leq \frac{1}{6}\left\|p_{x x}\right\|_{L^{2}}^{2}+C(t)\left\|p_{x}\right\|_{L^{2}}^{\gamma-1}\left\|q_{x}\right\|_{L^{2}}^{2} \\
& \leq \frac{1}{6}\left\|p_{x x}\right\|_{L^{2}}^{2}+C(t)\left(\left\|p_{x}\right\|_{L^{2}}^{2}+1\right)\left\|q_{x}\right\|_{L^{2}}^{2} .
\end{aligned}
$$

Substituting (4.7)-(4.10) into (4.6), we can show that

$$
\begin{aligned}
& \frac{\mathrm{d}}{\mathrm{d} t}\left(\left\|p_{x}\right\|_{L^{2}}^{2}+\left\|q_{x}\right\|_{L^{2}}^{2}\right)+\left\|p_{x x}\right\|_{L^{2}}^{2}+\varepsilon\left\|q_{x x}\right\|_{L^{2}} \\
\leq & C(t)\left(\left\|p p_{x}\right\|_{L^{2}}^{2}+\left\|p_{x}\right\|_{L^{2}}^{2}+1\right)\left(\left\|p_{x}\right\|_{L^{2}}^{2}+\left\|q_{x}\right\|_{L^{2}}^{2}\right)+C \varepsilon\left\|q_{x}\right\|_{L^{2}}^{2} .
\end{aligned}
$$

Applying Grönwall's inequality to (4.11) and using (4.2), (4.4) and (4.5), we can show that

$$
\left\|p_{x}\right\|_{L^{2}}^{2}+\left\|q_{x}\right\|_{L^{2}}^{2}+\int_{0}^{t}\left(\left\|p_{x x}\right\|_{L^{2}}^{2}+\varepsilon\left\|q_{x x}\right\|_{L^{2}}\right) \mathrm{d} \tau \leq C(t),
$$

where the constant $C(t)$ is independent of $\varepsilon$.

Step 4. When $3<\gamma \leq 4$, we can show that

$$
\begin{aligned}
\left|I_{3}\right| & \leq C\|p\|_{L^{\infty}}\left\|p_{x}\right\|_{L^{\infty}} \int_{0}^{1} p^{\gamma-3}\left|p_{x} \| q_{x}\right| \mathrm{d} x \\
& \leq C\|p\|_{L^{2}}^{\frac{1}{2}}\left\|p_{x}\right\|_{L^{2}}\left\|p_{x x}\right\|_{L^{2}}^{\frac{1}{2}}\left(\int_{0}^{1} p^{2(\gamma-3)}\left(p_{x}\right)^{2} \mathrm{~d} x\right)^{\frac{1}{2}}\left\|q_{x}\right\|_{L^{2}} \\
& \leq C(t)\left\|p_{x}\right\|_{L^{2}}\left\|p_{x x}\right\|_{L^{2}}^{\frac{1}{2}}\left(\left\|p p_{x}\right\|_{L^{2}}^{2}+\left\|p_{x}\right\|_{L^{2}}^{2}\right)^{\frac{1}{2}}\left\|q_{x}\right\|_{L^{2}} \\
& \leq \frac{1}{6}\left\|p_{x x}\right\|_{L^{2}}^{2}+C(t)\left\|p_{x}\right\|_{L^{2}}^{\frac{4}{3}}\left(\left\|p p_{x}\right\|_{L^{2}}^{2}+\left\|p_{x}\right\|_{L^{2}}^{2}\right)^{\frac{2}{3}}\left\|q_{x}\right\|_{L^{2}}^{\frac{4}{3}} \\
& \leq \frac{1}{6}\left\|p_{x x}\right\|_{L^{2}}^{2}+C(t)\left(\left\|p p_{x}\right\|_{L^{2}}^{2}+\left\|p_{x}\right\|_{L^{2}}^{2}\right)\left\|q_{x}\right\|_{L^{2}}^{2}+C(t)\left\|p_{x}\right\|_{L^{2}}^{4},
\end{aligned}
$$

and by (3.35), we have

$$
\begin{aligned}
\left|I_{4}\right| & \leq C\|p\|_{L^{\infty}}^{\gamma-1}\left\|p_{x x}\right\|_{L^{2}}\left\|q_{x}\right\|_{L^{2}} \\
& \leq C(t)\left(\left\|p p_{x}\right\|_{L^{2}}^{2}+\left\|p_{x}\right\|_{L^{2}}^{2}\right)^{\frac{1}{2}}\left\|p_{x x}\right\|_{L^{2}}\left\|q_{x}\right\|_{L^{2}} \\
& \leq \frac{1}{6}\left\|p_{x x}\right\|_{L^{2}}^{2}+C(t)\left(\left\|p p_{x}\right\|_{L^{2}}^{2}+\left\|p_{x}\right\|_{L^{2}}^{2}\right)\left\|q_{x}\right\|_{L^{2}}^{2} .
\end{aligned}
$$


Substituting (4.7), (4.8), (4.13) and (4.14) into (4.6), we get (4.11), and then (4.12). Step 5. When $\gamma>4$, using Young's inequality, we can show that

$$
\begin{aligned}
\left|I_{3}\right| & \leq \gamma(\gamma-1) \int_{0}^{1} p^{\frac{\gamma}{2}} p^{\frac{\gamma}{2}-2}\left(p_{x}\right)^{2}\left|q_{x}\right| \mathrm{d} x \\
& \leq \gamma(\gamma-1)\left(\int_{0}^{1} p^{\gamma-4}\left(p_{x}\right)^{2} \mathrm{~d} x\right)^{\frac{1}{2}}\|p\|_{L^{\infty}}^{\frac{\gamma}{2}}\left\|p_{x}\right\|_{L^{\infty}}\left\|q_{x}\right\|_{L^{2}} \\
& \leq C\left(\int_{0}^{1} p^{\gamma-1}\left(p_{x}\right)^{2} \mathrm{~d} x+\left\|p_{x}\right\|_{L^{2}}^{2}\right)^{\frac{1}{2}}\|p\|_{L^{\infty}}^{\frac{\gamma}{2}}\left\|p_{x}\right\|_{L^{\infty}}\left\|q_{x}\right\|_{L^{2}} .
\end{aligned}
$$

Since $p \geq 0$ and $\left.p\right|_{x=0}=0$, we can show that

$$
\begin{aligned}
p^{\gamma} & =\gamma \int_{0}^{x} p^{\gamma-1} p_{y} \mathrm{~d} y \leq \gamma\left(\int_{0}^{1} p^{\gamma-1}\left(p_{x}\right)^{2} \mathrm{~d} x\right)^{\frac{1}{2}}\left(\int_{0}^{1} p^{\gamma-1} \mathrm{~d} x\right)^{\frac{1}{2}} \\
& \leq \gamma\left(\int_{0}^{1} p^{\gamma-1}\left(p_{x}\right)^{2} \mathrm{~d} x\right)^{\frac{1}{2}}\left(\int_{0}^{1} p^{\gamma} \mathrm{d} x\right)^{\frac{\gamma-1}{2 \gamma}} \leq C\left(\int_{0}^{1} p^{\gamma-1}\left(p_{x}\right)^{2} \mathrm{~d} x\right)^{\frac{1}{2}},
\end{aligned}
$$

where we used (4.2). This implies

$$
\|p\|_{L^{\infty}}^{\frac{\gamma}{2}} \leq C\left(\int_{0}^{1} p^{\gamma-1}\left(p_{x}\right)^{2} \mathrm{~d} x\right)^{\frac{1}{4}} .
$$

Substituting the above estimate into (4.15) and invoking interpolation inequality, we deduce

$$
\begin{aligned}
\left|I_{3}\right| & \leq C\left(\int_{0}^{1} p^{\gamma-1}\left(p_{x}\right)^{2} \mathrm{~d} x+\left\|p_{x}\right\|_{L^{2}}^{2}\right)^{\frac{3}{4}}\left\|p_{x}\right\|_{L^{2}}^{\frac{1}{2}}\left\|p_{x x}\right\|_{L^{2}}^{\frac{1}{2}}\left\|q_{x}\right\|_{L^{2}} \\
& \leq \frac{1}{6}\left\|p_{x x}\right\|_{L^{2}}^{2}+C\left(\int_{0}^{1} p^{\gamma-1}\left(p_{x}\right)^{2} \mathrm{~d} x+\left\|p_{x}\right\|_{L^{2}}^{2}\right)\left\|p_{x}\right\|_{L^{2}}^{\frac{2}{3}}\left\|q_{x}\right\|_{L^{2}}^{\frac{4}{3}} \\
& \leq \frac{1}{6}\left\|p_{x x}\right\|_{L^{2}}^{2}+C\left(\int_{0}^{1} p^{\gamma-1}\left(p_{x}\right)^{2} \mathrm{~d} x+\left\|p_{x}\right\|_{L^{2}}^{2}\right)\left(\left\|p_{x}\right\|_{L^{2}}^{2}+\left\|q_{x}\right\|_{L^{2}}^{2}\right) .
\end{aligned}
$$

Lastly, using (3.50), we can show that

$$
\begin{aligned}
\left|I_{4}\right| & \leq\|p\|_{L^{\infty}}^{\gamma-1}\left\|p_{x x}\right\|_{L^{2}}\left\|q_{x}\right\|_{L^{2}} \\
& \leq C\left(\int_{0}^{1} p^{\gamma-1}\left(p_{x}\right)^{2} \mathrm{~d} x+\left\|p_{x}\right\|_{L^{2}}^{2}\right)^{\frac{1}{2}}\left\|p_{x x}\right\|_{L^{2}}\left\|q_{x}\right\|_{L^{2}} \\
& \leq \frac{1}{6}\left\|p_{x x}\right\|_{L^{2}}^{2}+C\left(\int_{0}^{1} p^{\gamma-1}\left(p_{x}\right)^{2} \mathrm{~d} x+\left\|p_{x}\right\|_{L^{2}}^{2}\right)\left\|q_{x}\right\|_{L^{2}}^{2} .
\end{aligned}
$$


Substituting (4.7), (4.8), (4.16) and (4.17) into (4.6), we get

$$
\begin{aligned}
& \frac{\mathrm{d}}{\mathrm{d} t}\left(\left\|p_{x}\right\|_{L^{2}}^{2}+\left\|q_{x}\right\|_{L^{2}}^{2}\right)+\left\|p_{x x}\right\|_{L^{2}}^{2}+\varepsilon\left\|q_{x x}\right\|_{L^{2}} \\
\leq & C\left(\int_{0}^{1} p^{\gamma-1}\left(p_{x}\right)^{2} \mathrm{~d} x+\left\|p_{x}\right\|_{L^{2}}^{2}+1\right)\left(\left\|p_{x}\right\|_{L^{2}}^{2}+\left\|q_{x}\right\|_{L^{2}}^{2}\right)+C \varepsilon\left\|q_{x}\right\|_{L^{2}}^{2} .
\end{aligned}
$$

Applying Grönwall's inequality and invoking (4.2), (4.4) and (4.5), we arrive at (4.12).

It should be stressed that for all $\gamma \geq 2$, the constants in the energy estimates (4.2), (4.4), (4.5) and (4.12) are independent of $\varepsilon$ and remain finite for any finite time. Therefore, the energy estimates stated in Theorem 1.3 are established.

\subsection{Diffusion limit}

Let $\left(p^{\varepsilon}, q^{\varepsilon}\right)$ and $\left(p^{0}, q^{0}\right)$ denote, respectively, the solution to (4.1) when $\varepsilon>0$ and $\varepsilon=0$, with the same initial data, and let $(P, Q)=\left(p^{\varepsilon}-p^{0}, q^{\varepsilon}-q^{0}\right)$. Then $(P, Q)$ satisfies

$$
\begin{cases}P_{t}-\left(p^{0} Q\right)_{x}-\left(P q^{\varepsilon}\right)_{x}=P_{x x}, & x \in(0,1), \quad t>0, \\ Q_{t}-\left[\left(p^{\varepsilon}\right)^{\gamma}-\left(p^{0}\right)^{\gamma}\right]_{x}=\varepsilon q_{x x}^{\varepsilon}+2 \varepsilon q^{\varepsilon} q_{x}^{\varepsilon}, & x \in(0,1), \quad t>0, \\ (P, Q)(x, 0)=(0,0), & x \in(0,1), \\ \left.P\right|_{x=0,1}=0, & t>0 .\end{cases}
$$

Using the energy estimates established in Subsection 4.1, we can show that

$$
\|P(t)\|_{L^{2}}^{2}+\|Q(t)\|_{L^{2}}^{2} \leq C(t) \varepsilon,
$$

where $C(t)>0$ is independent of $\varepsilon$ and remains finite for any finite time. The argument leading to (4.18) is in the same spirit of [38], and we omit the details to simplify the presentation. This completes the proof of Theorem 1.3.

\section{Acknowledgements}

The authors would like to thank the anonymous referees for constructive comments and suggestions which help improve the quality of the paper. Z.-F. Feng was partially supported by China Scholarship Council (No. 201906150159). J. Xu was partially supported by China Scholarship Council (No. 201906150101) and National Natural Science Foundation of China (No. 11971176 and No. 11871226). L. Xue was partially supported by Fundamental Research Funds for the Central Universities of China (No. 3072020CFT2402). K. Zhao was partially supported by Simons Foundation Collaboration Grant for Mathematicians (No. 413028). 


\section{References}

[1] W. Alt and D. A. Lauffenburger, Transient behavior of a chemotaxis system modeling certain types of tissue inflammation, J. Math. Biol., 24 (1987), pp. 691-722.

[2] D. Balding and D. L. S. McElwain, A mathematical model of tumour-induced capillary growth, J. Theor. Biol., 114 (1985), pp. 53-73.

[3] J. A. Carrillo, J. Y. Li and Z. A. Wang, Boundary spike-layer solutions of the singular Keller-Segel system: existence and stability, Proc. London Math. Soc., 122 (2021), pp. $42-68$.

[4] F. W. Dahlquist, P. Lovely and D. E. Jr Koshland, Quantitative analysis of bacterial migration in chemotaxis, Nature, New Biol., 236 (1972), pp. 120-123.

[5] J. Fan and K. Zhao, Blow up criterion for a hyperbolic-parabolic system arising from chemotaxis, J. Math. Anal. Appl., 394 (2012), pp. 687-695.

[6] M. A. Fontelos, A. Friedman and B. Hu, Mathematical analysis of a model for the initiation of angiogenesis, SIAM J. Math. Anal., 33 (2002), pp. 1330-1355.

[7] J. Guo, J. Xiao, H. Zhao and C. Zhu, Global solutions to a hyperbolic-parabolic coupled system with large initial data, Acta Math. Sci. Ser. B (Engl. Ed.), 29 (2009), pp. 629-641.

[8] Q. Hou, Z. A. Wang and K. Zhao, Boundary layer problem on a hyperbolic system arising from chemotaxis, J. Differential Equations, 261 (2016), pp. 5035-5070.

[9] Q. Hou, C. Liu, Y. Wang and Z. A. Wang, Stability of boundary layers for a viscous hyperbolic system arising from chemotaxis: one dimensional case, SIAM J. Math. Anal., 50 (2018), pp. 3058-3091.

[10] Q. Hou and Z. A. Wang, Convergence of boundary layers for the Keller-Segel system with singular sensitivity in the half-plane, J. Math. Pures. Appl., 130 (2019), pp. $251-287$.

[11] F. Huang, P. Marcati and R. Pan, Convergence to the Barenblatt solutions for the compressible Euler equations with damping and vacuum, Arch. Ration. Mech. Anal., 176 (2005), pp. 1-24.

[12] F. Huang, R. Pan and Z. Wang, $L^{1}$ Convergence to the Barenblatt solution for compressible Euler equations with damping, Arch. Ration. Mech. Anal., 200 (2011), pp. 665-689.

[13] H.Y. Jin, J. Li and Z. A. Wang, Asymptotic stability of traveling waves of a chemotaxis model with singular sensitivity, J. Differential Equations, 255 (2013), pp. 193219.

[14] Y. V. Kalinin, L. Jiang, Y. Tu and M. Wu, Logarithmic sensing in Escherichia coli bacterial chemotaxis, Biophysical J., 96 (2009), pp. 2439-2448.

[15] E. F. Keller and L. A. Segel, Traveling bands of chemotactic bacteria: a theoretical analysis, J. Theor. Biol., 30 (1971), pp. 235-248.

[16] H. A. Levine and B. D. Sleeman, A system of reaction diffusion equations arising in the theory of reinforced random walks, SIAM J. Appl. Math., 57 (1997), pp. 683-730.

[17] H. A. Levine, B. D. Sleeman and M. Nilsen-Hamilton, A mathematical model for the 
roles of pericytes and macrophages in the initiation of angiogenesis. I. The role of protease inhibitors in preventing angiogenesis, Math. Biosci., 168 (2000), pp. 71-115.

[18] D. Li, R. Pan and K. Zhao, Quantitative decay of a one-dimensional hybrid chemotaxis model with large data, Nonlinearity, 28 (2015), pp. 2181-2210.

[19] H. Li and K. Zhao, Initial-boundary value problems for a system of hyperbolic balance laws arising from chemotaxis, J. Differential Equations, 258 (2015), pp. 302-338.

[20] T. Li, R. Pan and K. Zhao, Global dynamics of a hyperbolic-parabolic model arising from chemotaxis, SIAM J. Appl. Math., 72 (2012), pp. 417-443.

[21] T. Li and Z. A. Wang, Nonlinear stability of traveling waves to a hyperbolic-parabolic system modeling chemotaxis, SIAM J. Appl. Math., 7 (2009), pp. 1522-1541.

[22] T. Li, and Z. A. Wang, Nonlinear stability of large amplitude viscous shock waves of a generalized hyperbolic-parabolic system arising in chemotaxis, Math. Models Methods Appl. Sci., 20 (2010), pp. 1967-1998.

[23] T. Li, and Z. A. Wang, Asymptotic nonlinear stability of traveling waves to conservation laws arising from chemotaxis, J. Differential Equations, 250 (2011), pp. $1310-1333$.

[24] T. Li, and Z. A. Wang, Steadily propagating waves of a chemotaxis model, Math. Biosci., 240 (2012), pp. 161-168.

[25] P. L. Lions, Mathematical Topics in Fluid Mechanics, Vol. I: Incompressible Models, Oxford Lecture Ser. Math. Appl., Oxford University Press, Oxford, 1996.

[26] P. L. Lions, Mathematical Topics in Fluid Mechanics, Vol. II: Compressible Models, Oxford Lecture Ser. Math. Appl., Oxford University Press, Oxford, 1998.

[27] Z. Luo, Local existence of classical solutions to the two-dimensional viscous compressible flows with vacuum, Commun. Math. Sci., 10 (2012), pp. 527-554.

[28] V. Martinez, Z. A. Wang and K. Zhao, Asymptotic and viscous stability of largeamplitude solutions of a hyperbolic system arising from biology, Indiana Univ. Math. J., 67 (2018), pp. 1383-1424.

[29] H. G. Othmer and A. Stevens, Aggregation, blowup, and collapse: the ABCs of taxis in reinforced random walks, SIAM J. Appl. Math., 57 (1997), pp. 1044-1081.

[30] H. Peng, Z. A. Wang, K. Zhao and C. Zhu, Boundary layers and stabilization of the singular Keller-Segel model, Kinet. Relat. Models, 11 (2018), pp. 1085-1123.

[31] H. Peng, H. Wen and C. Zhu, Global well-posedness and zero diffusion limit of classical solutions to 3D conservation laws arising in chemotaxis, Z. Angew. Math. Phys., 65 (2014), pp. 1167-1188.

[32] L. G. Rebholz, D. Wang, Z. A. Wang, C. Zerfas and K. Zhao, Initial boundary value problems for a system of parabolic conservation laws arising from chemotaxis in multi-dimensions, Discrete Contin. Dyn. Syst. A, 39 (2019), pp. 3789-3838.

[33] Y. Tao, L. Wang and Z. A. Wang, Large-time behavior of a parabolic-parabolic chemotaxis model with logarithmic sensitivity in one dimension, Discrete Contin. Dyn. Syst., Ser. B, 18 (2013), pp. 821-845.

[34] D.-H. Wang and C. Yu, Global weak solution and large-time behavior for the compressible flow of liquid crystals, Arch. Ration. Mech. Anal., 204 (2012), pp. 881-915. 
[35] Z. A. Wang, Z. Xiang and P. Yu, Asymptotic dynamics on a singular chemotaxis system modeling onset of tumor angiogenesis, J. Differential Equations, 260 (2016), pp. 2225-2258.

[36] Z. A. Wang and K. Zhao, Global dynamics and diffusion limit of a parabolic system arising from repulsive chemotaxis, Commun. Pure Appl. Anal., 12 (2013), pp. 30273046.

[37] M. Zhang and C. Zhu, Global existence of solutions to a hyperbolic-parabolic system, Proc. Amer. Math. Soc., 135 (2007), pp. 1017-1027.

[38] N. Zhu, Z. R. Liu, V. Martinez and K. Zhao, Global Cauchy problem of a system of parabolic conservation laws arising from a Keller-Segel type chemotaxis model, SIAM J. Math. Anal., 50 (2018), pp. 5380-5425. 